Next Generation Hydrogen Station Composite Data Products: All Stations (Retail and Non-Retail Combined) Data through Quarter 3 of 2016

Sam Sprik, Jennifer Kurtz, Chris Ainscough, Genevieve Saur, Michael Peters, and Matthew Jeffers January 20, 2017 

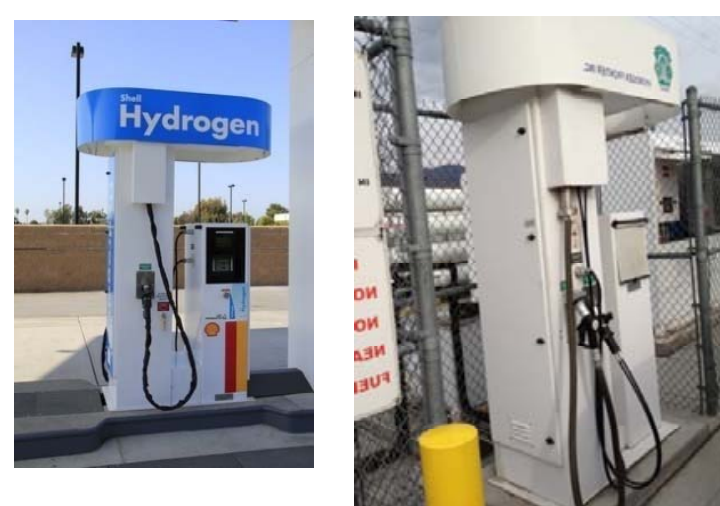

- California Energy Commission

- California State University Los Angeles

- First Element

- Gas Technology Institute

- Linde
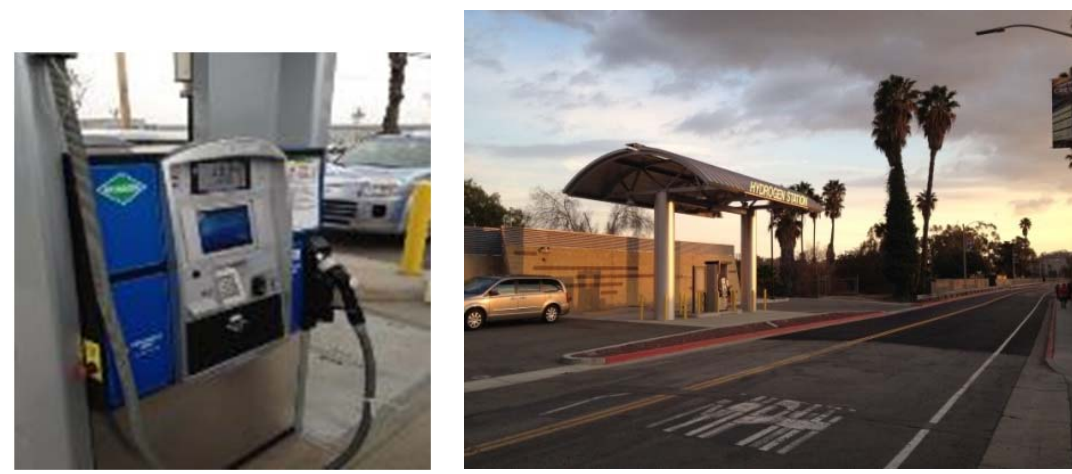

- H2 Frontier

- Proton OnSite

- Shell

- IPHE and HySUT

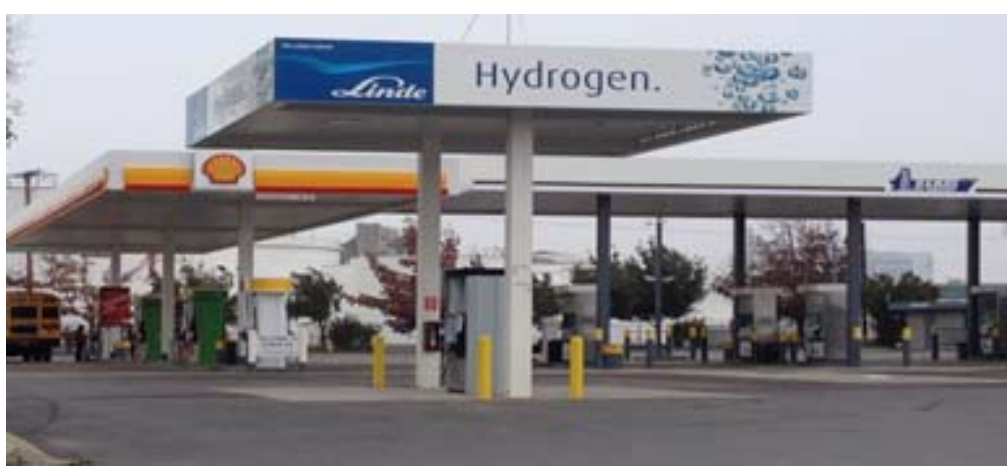




\section{Analysis Categories}

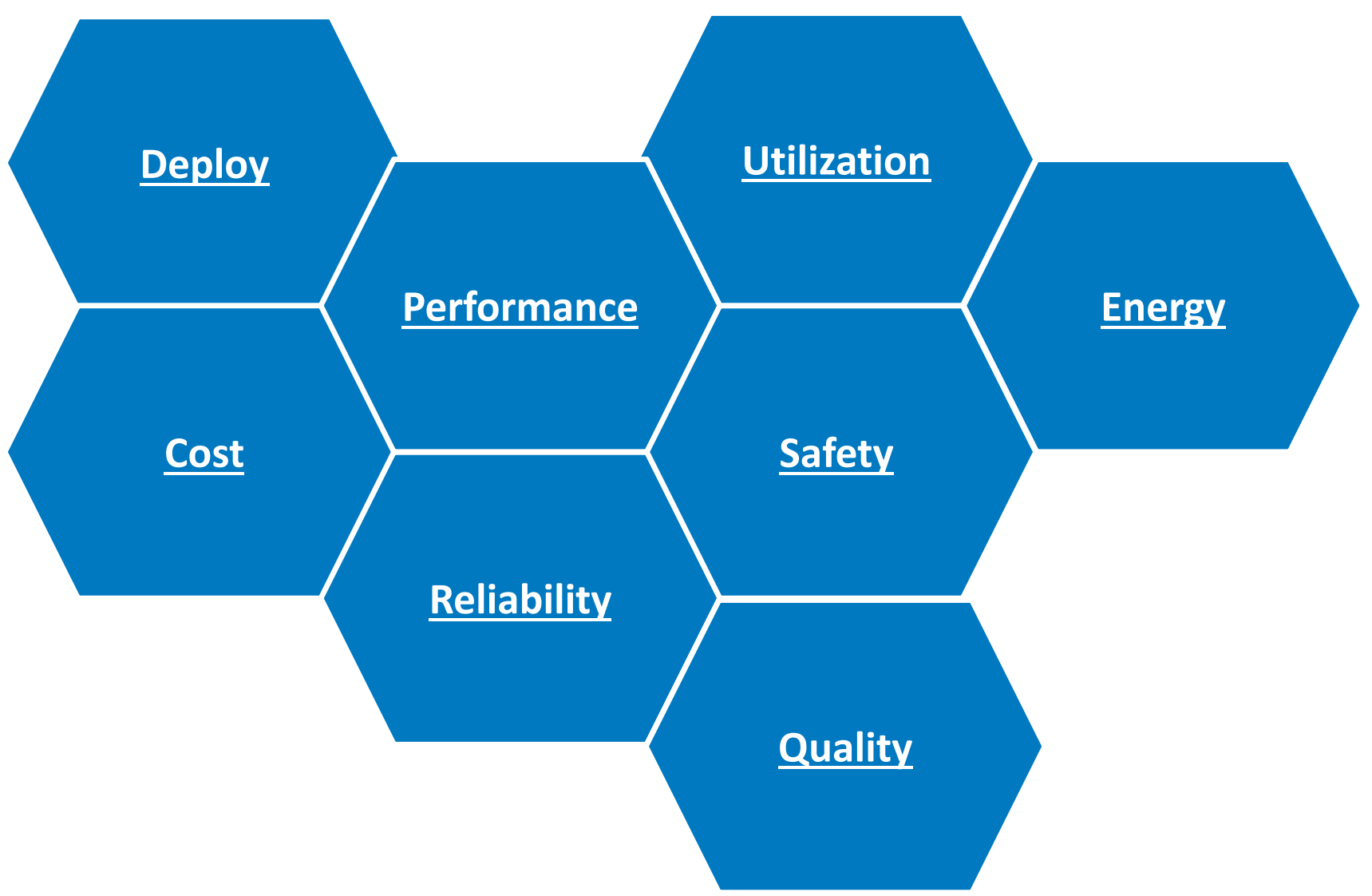


Deployment 


\section{Cumulative Number of Stations}

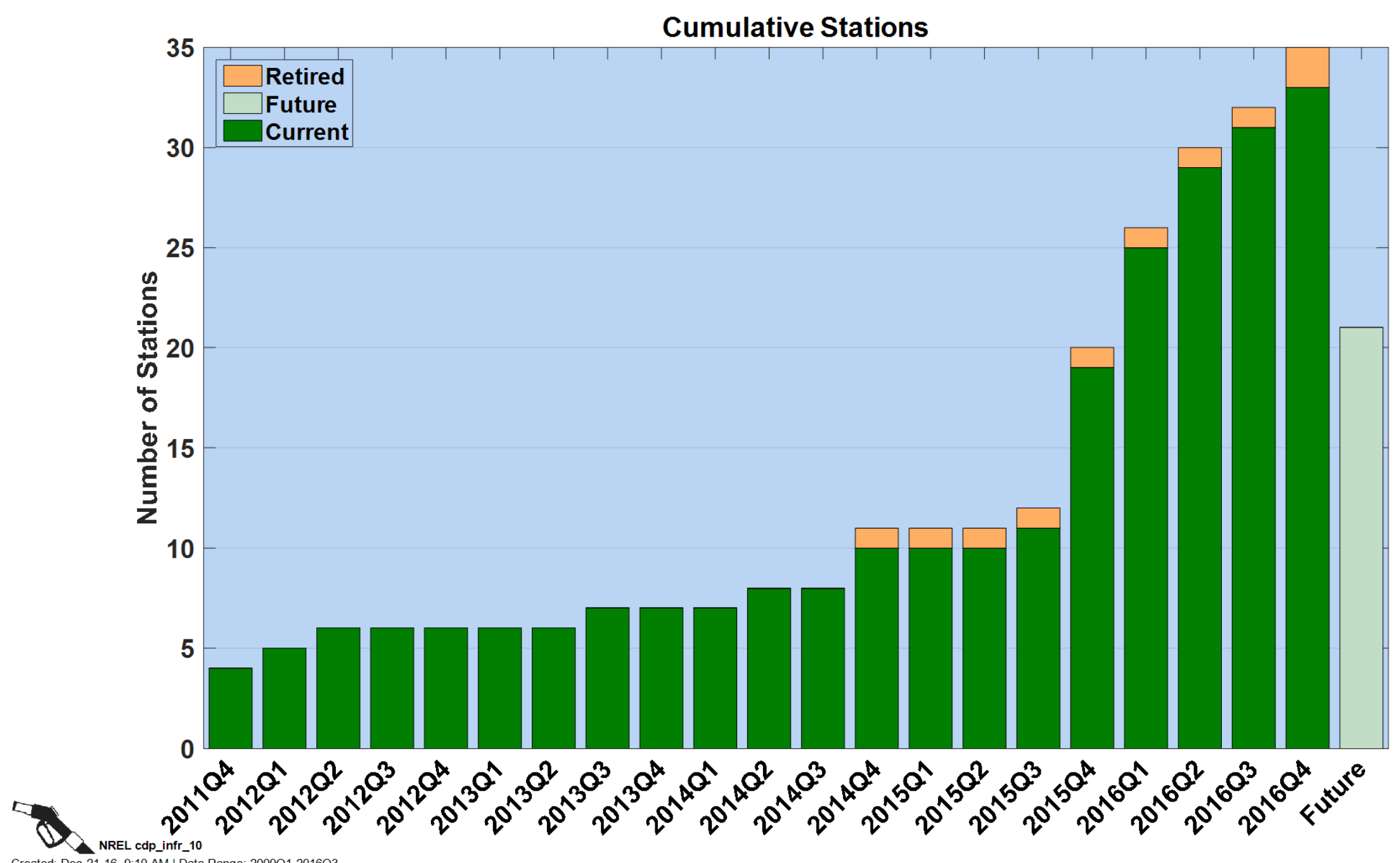




\section{Hydrogen Stations by Type}

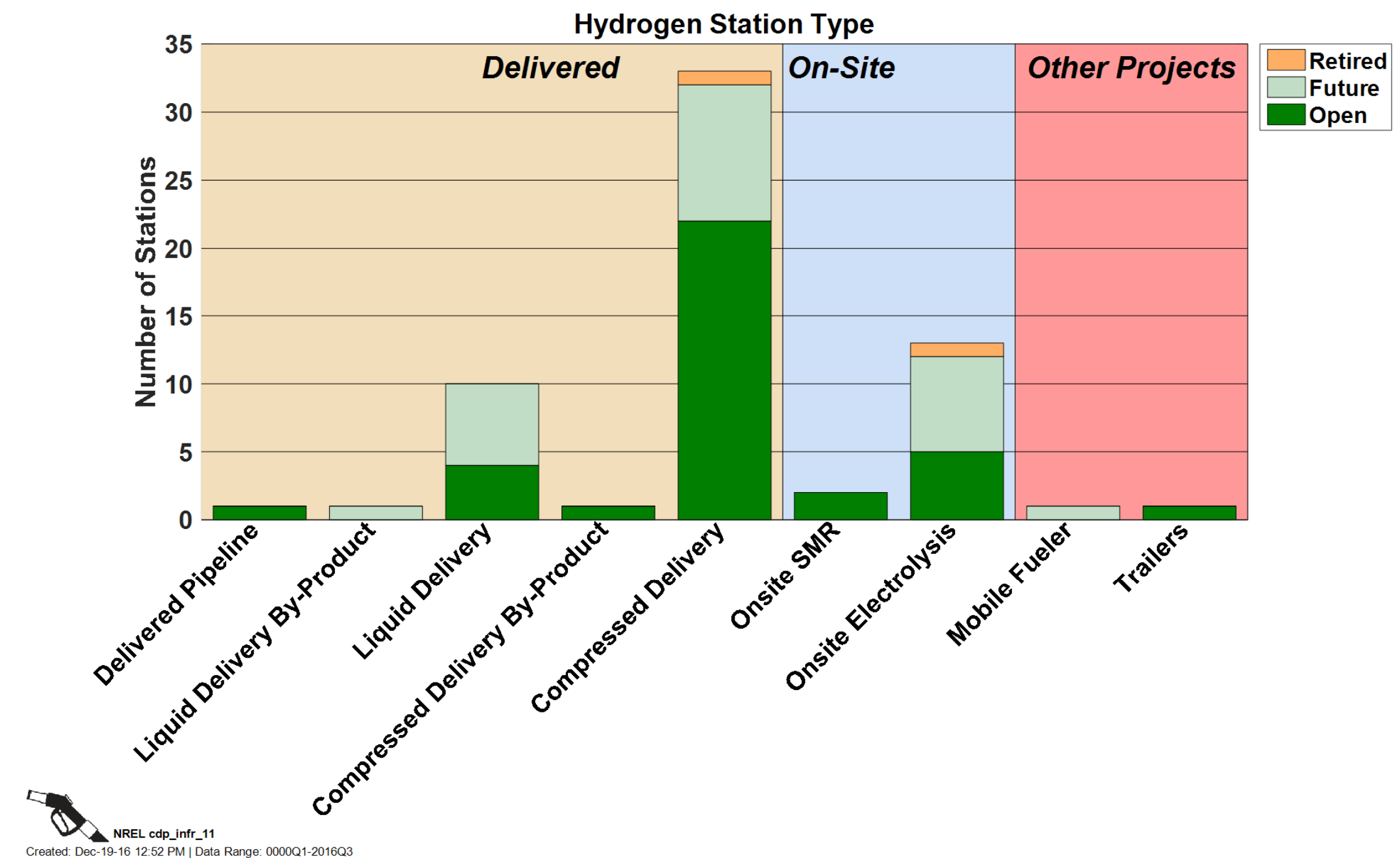




\section{Hydrogen Station Timeline}

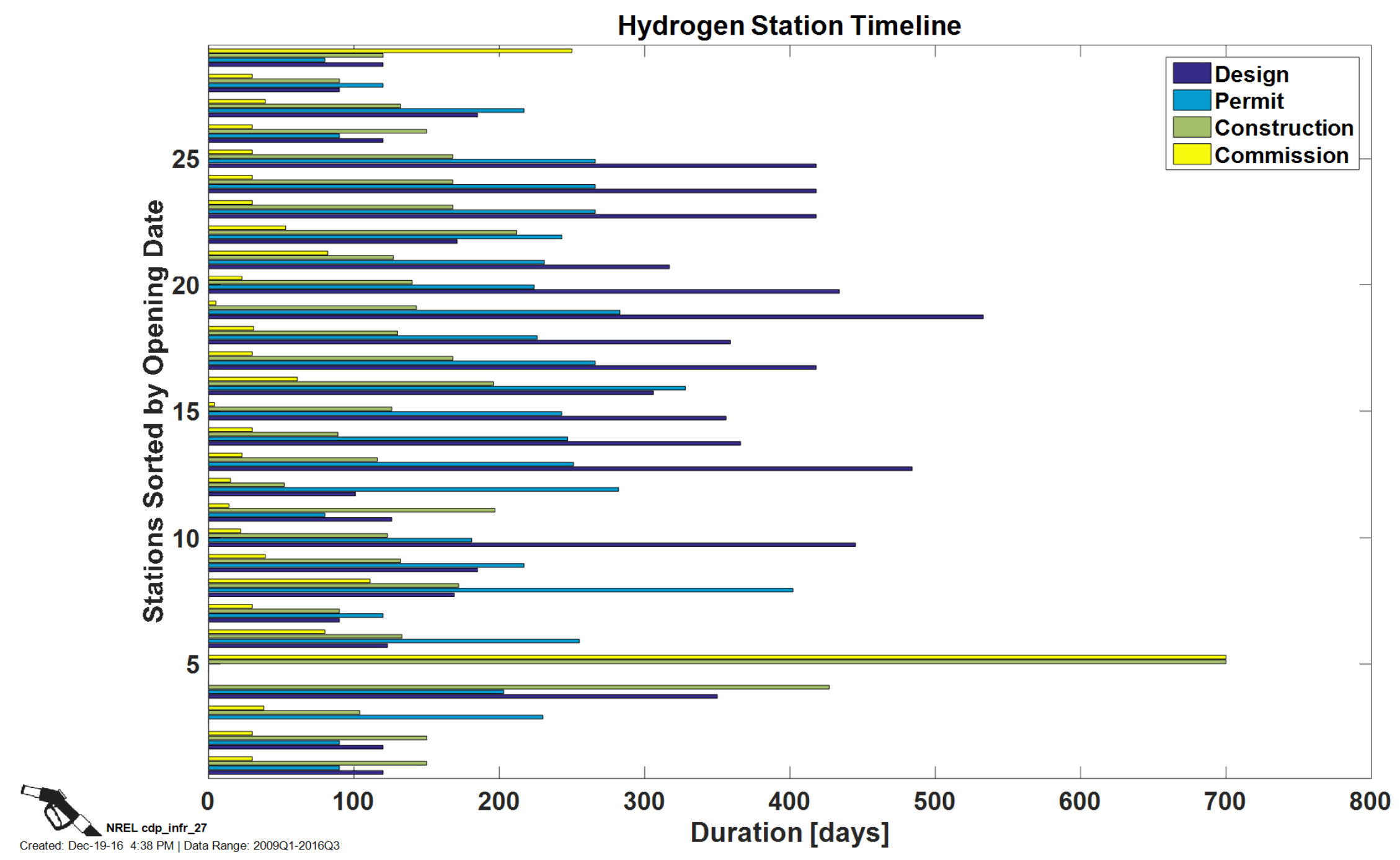




\section{Safety}




\section{Safety (and Maintenance) Learnings}

\section{From Safety Reports Template}

- Alarms not communicated

- Breakaway leak

- Check compressor oil filter

- Check integrity of delivered equipment

- Compressor leaking at startup normal?

- Does isolated leak need to shut down station?

- Electrical glitch

- Estop activated after hearing escaping gas-nitrogen

- Estop activated when nozzle stuck on car

- Estop activated without cause

- Estop flooded prevented restart

- False Alarm - No Fire

- Fill and leak check together caused shutdown - false leak alarm

- Filter to catch scrap from material processing

- Forgot to turn back on after maintenance

- Freezing and thawing caused moisture in communication connector

- Frozen cooling block - defrost

- HTO sensor fault
- $\quad$ Heat trace short caused false fire alarm

- Heavy rain triggered fire alarm

- Hose vent failure - nozzle stuck on car

- Loose wire intermittent problems

- $\quad$ Loud popping could be relief valve

- Mass balance alarm bug

- Mass balance alarm caused by high ambient temperature

- $\quad$ Power Issue - 3 Phase

- Predict service life better

- $\quad$ Proper installation prevents leaks

- Rain on sensor causing alarm

- Regular inspection of compressor valves

- Regular leak checks

- Regular station inspection

- Reset

- $\quad$ Spider web obscuring sensor

- Thermocouple failure shutdown station

- Vibration from normal activity shutdown dispenser

- Vibration isolation 


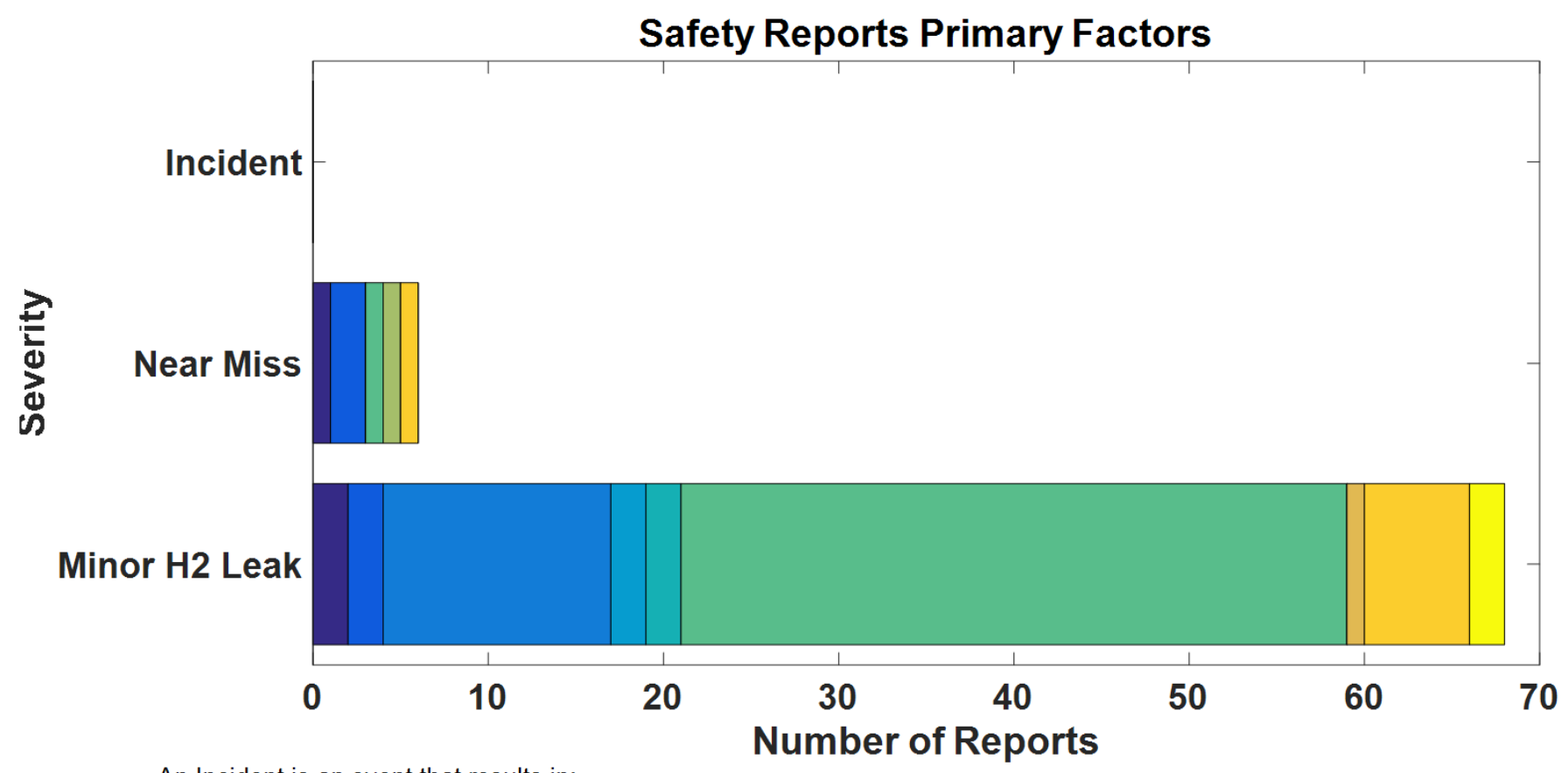

Component Failure

Design Flaw

Environment (Weather, Power Disrup Inadequate Training, Protocol, SOP Inadequate/ Non-working Equipment Maintenance Required Mischief/Vandalism New Equipment Materials Not Defined

Vibration

An Incident is an event that results in:

- a lost time accident and/or injury to personnel

- damage/unplanned downtime for project equipment, facilities or property

- impact to the public or environment

- any hydrogen release that unintentionally ignites

- release of any volatile, hydrogen containing compound (including the hydrocarbons used as common fuels) A Near Miss is:

- an event that under slightly different circumstances could have become an incident

- any hydrogen release sufficient to sustain a flame if ignited

A Minor H2 Leak is:

- an unplanned hydrogen release insufficient to sustain a flame, and does not accumulate in sufficient quantity to ignite 


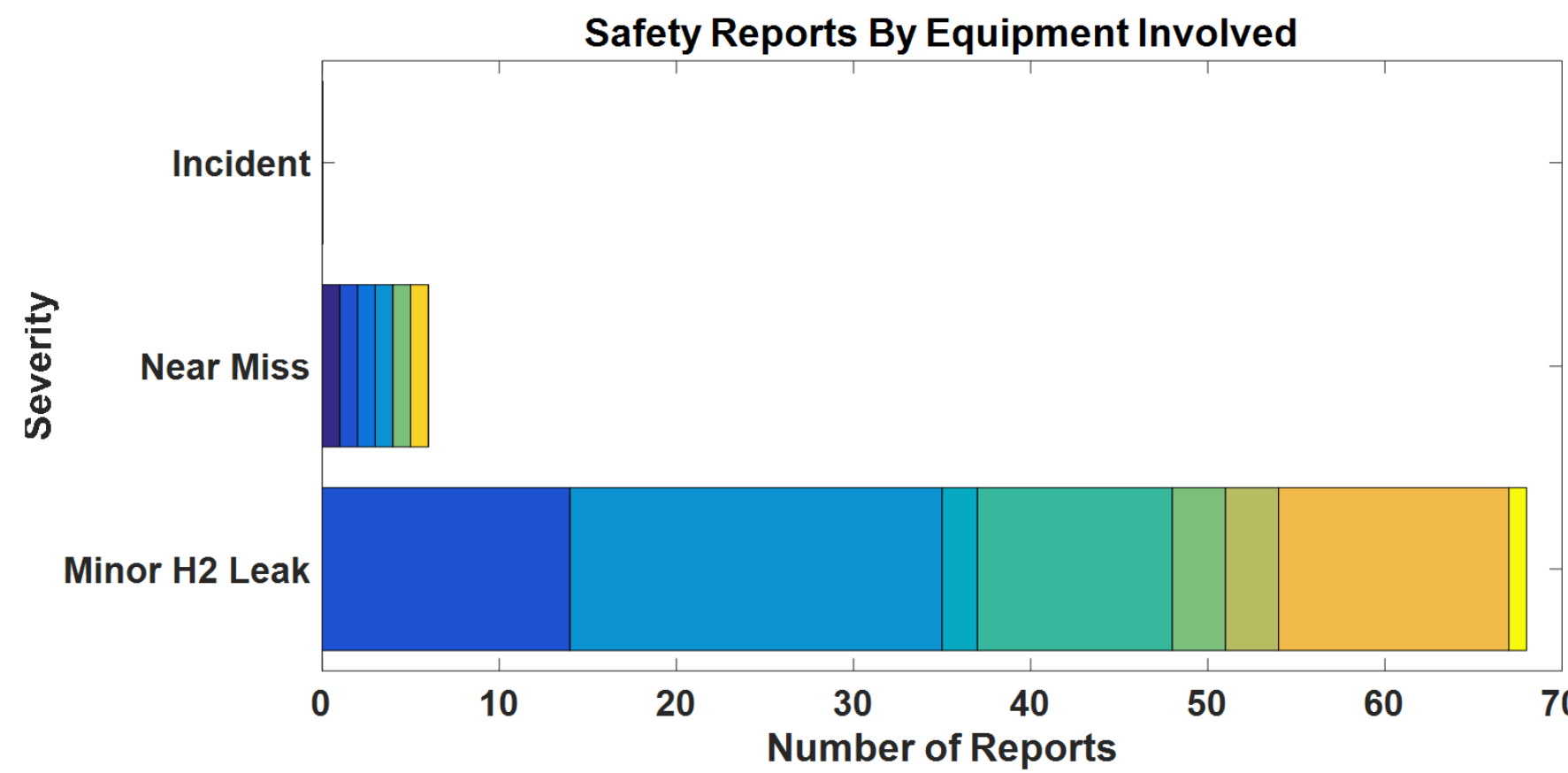

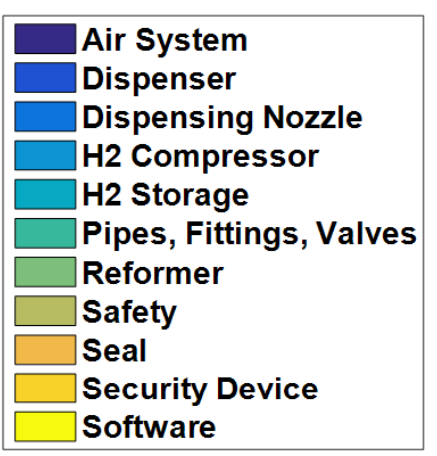

An Incident is an event that results in:

- a lost time accident and/or injury to personnel

- damage/unplanned downtime for project equipment, facilities or property

- impact to the public or environment

- any hydrogen release that unintentionally ignites A Near Miss is:

- release of any volatile, hydrogen containing compound (including the hydrocarbons used as common fuels)

- an event that under slightly different circumstances could have become an incident

- any hydrogen release sufficient to sustain a flame if ignited

A Minor H2 Leak is:

(1) - an

- an unplanned hydrogen release insufficient to sustain a flame, and does not accumulate in sufficient quantity to ignite 


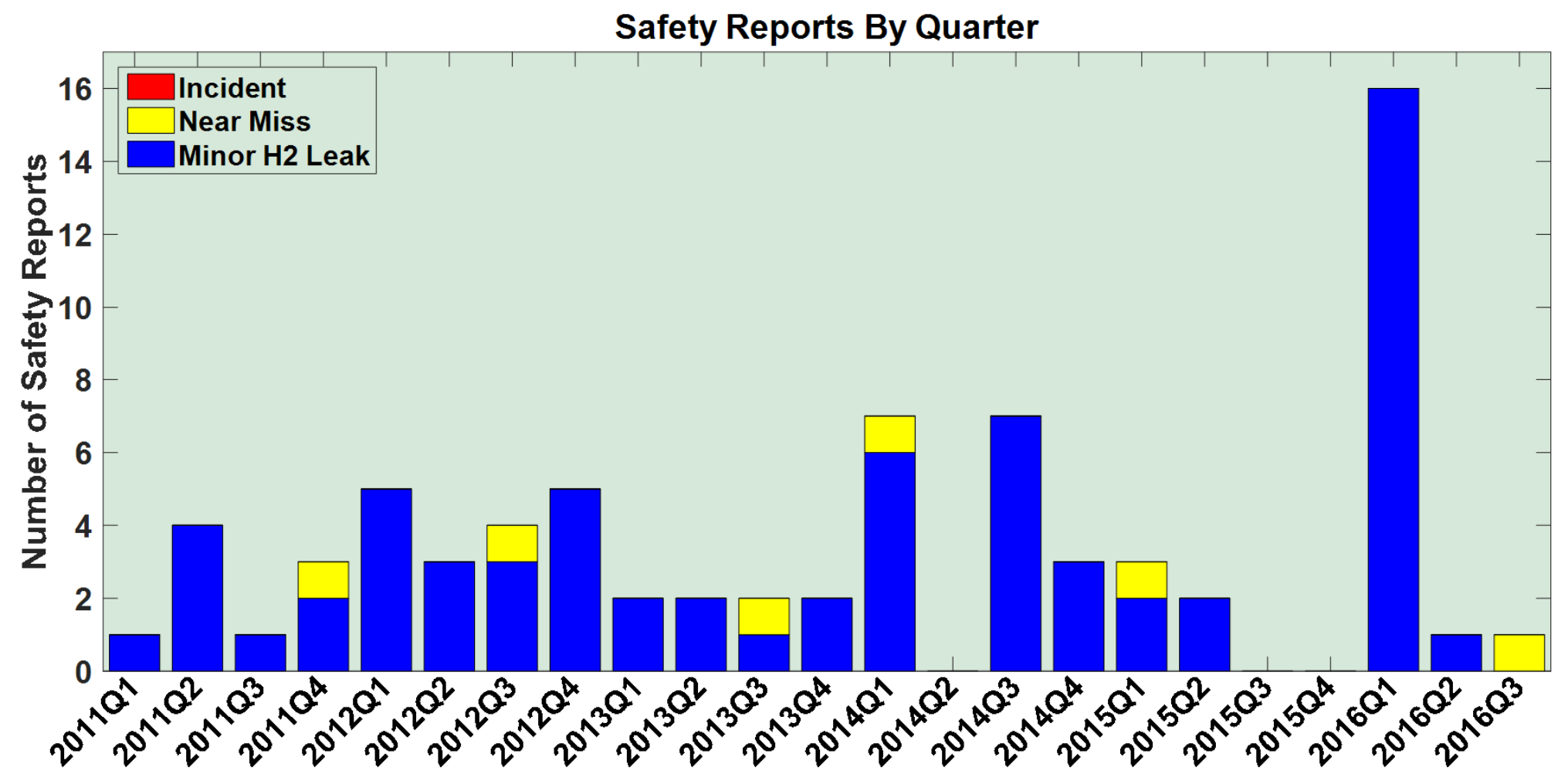

An Incident is an event that results in:

- a lost time accident and/or injury to personnel

- damage/unplanned downtime for project equipment, facilities or property

- impact to the public or environment

- any hydrogen release that unintentionally ignites

- release of any volatile, hydrogen containing compound (including the hydrocarbons used as common fuels)

(1) NREL cdp_infr_33

Created: Dec-12-16 2:00 PM | Data Range: 2008Q3-2016Q3 A Near Miss is:

- an event that under slightly different circumstances could have become an incident

- any hydrogen release sufficient to sustain a flame if ignited

A Minor H2 Leak is: 


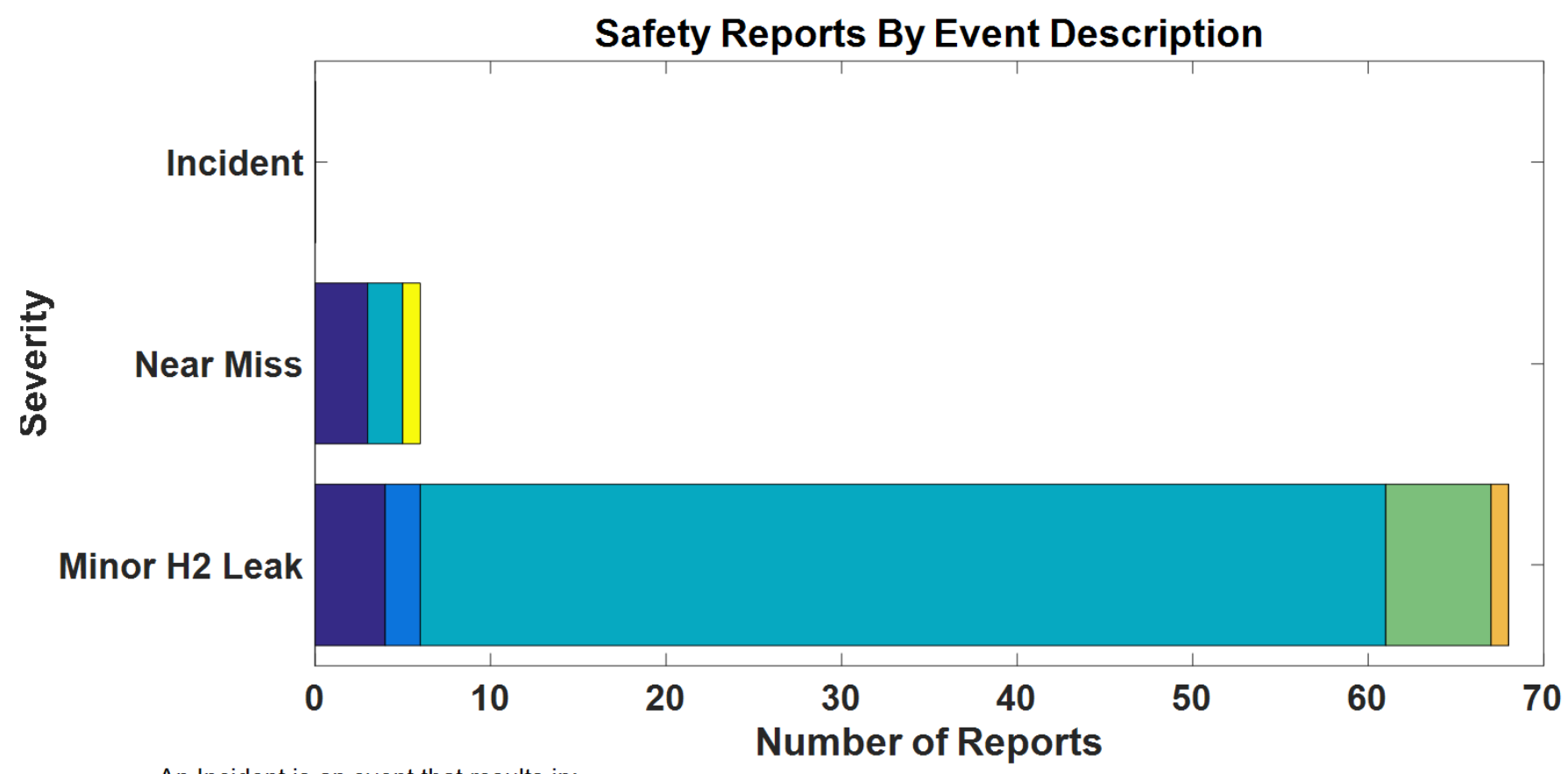

Equipment Malfunction H2 Release - Accumulation H2 Release - No Accumulation H2 Release-Minor Non-H2 Release Vandalism

An Incident is an event that results in:

- a lost time accident and/or injury to personnel

- damage/unplanned downtime for project equipment, facilities or property

- impact to the public or environment

- any hydrogen release that unintentionally ignites

- release of any volatile, hydrogen containing compound (including the hydrocarbons used as common fuels) A Near Miss is:

- an event that under slightly different circumstances could have become an incident

- any hydrogen release sufficient to sustain a flame if ignited

A Minor $\mathrm{H} 2$ Leak is:

- an unplanned hydrogen release insufficient to sustain a flame, and does not accumulate in sufficient quantity to ignite 


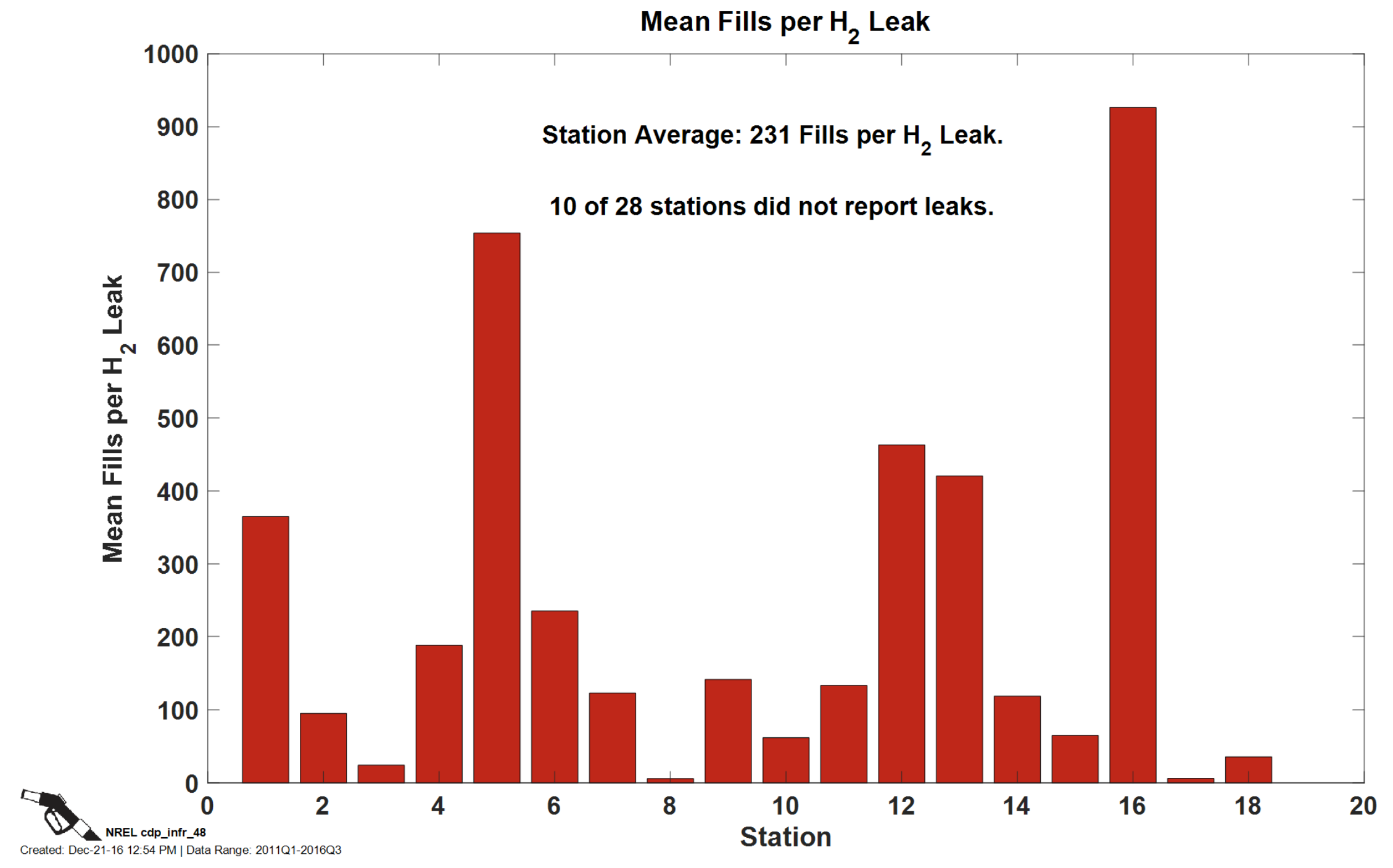


CDP-INFR-54

Mean Hydrogen Dispensed per Hydrogen Leak

Mean $\mathrm{H}_{2}$ Dispensed (kg) per Leak

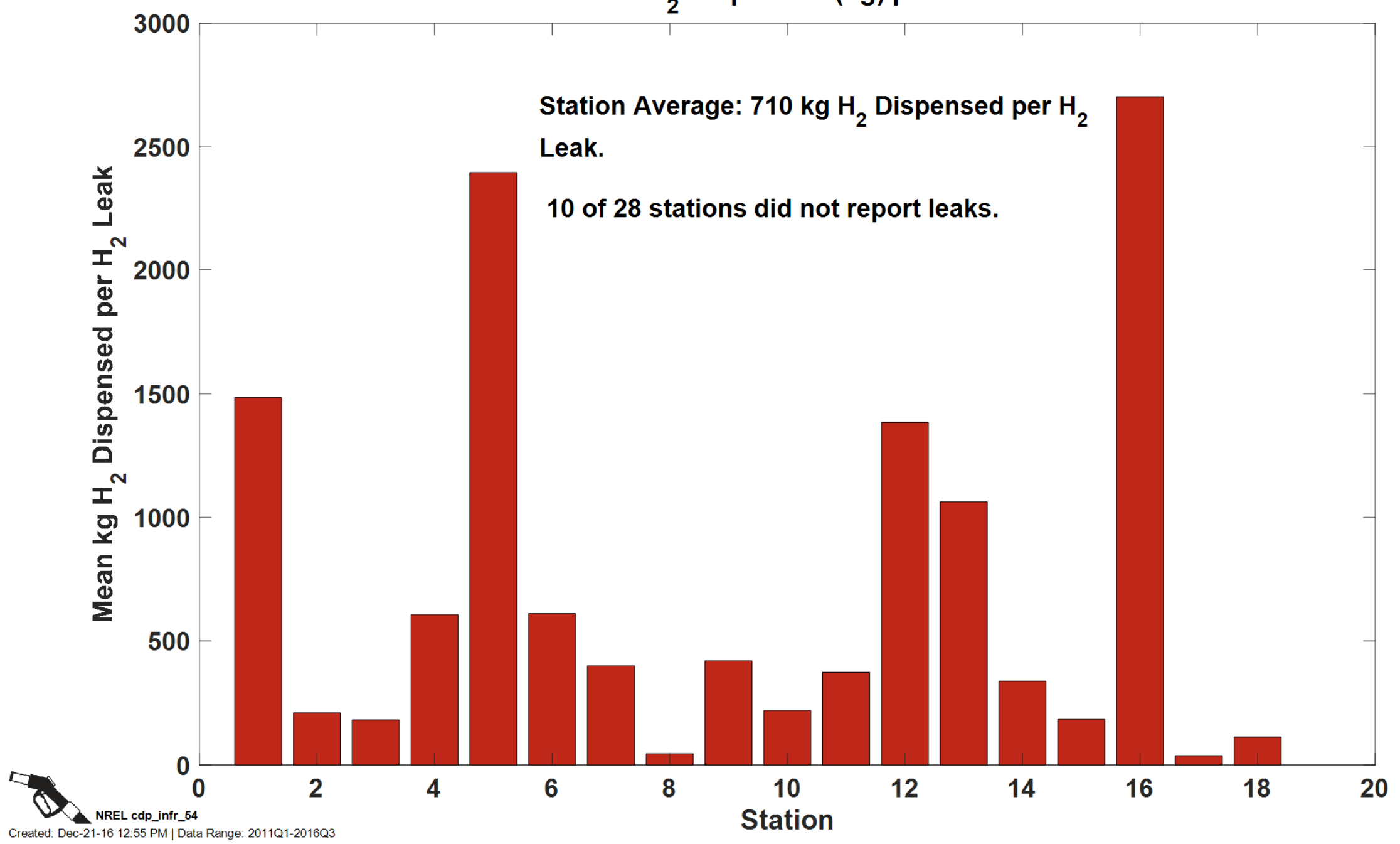


Maintenance and Reliability 


\section{Maintenance by Equipment Type}
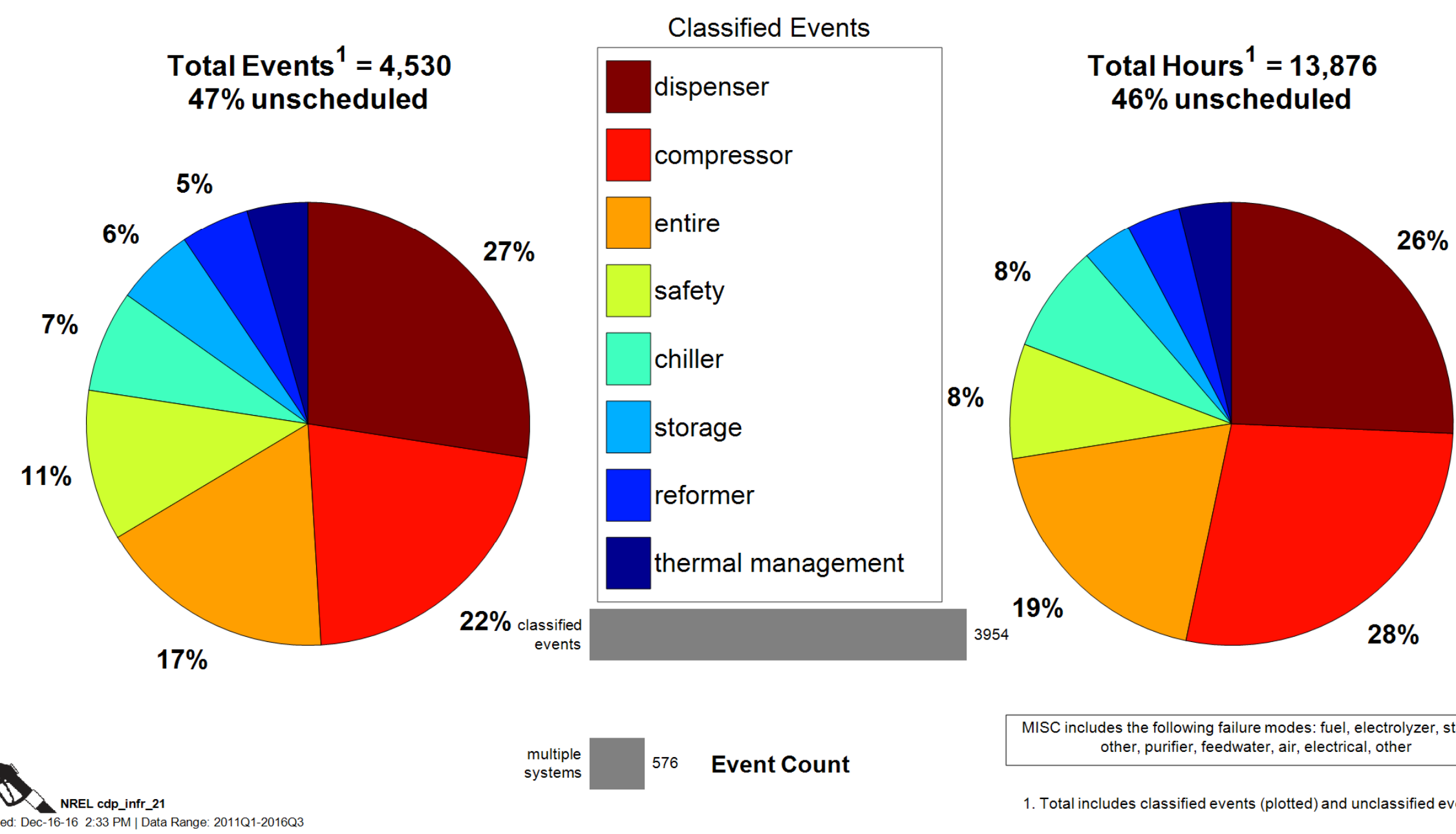
MISC includes the following failure modes: fuel, electrolyzer, station
other, purifier, feedwater, air, electrical, other

1. Total includes classified events (plotted) and unclassified events. 


\section{Maintenance Labor Hours per Event}

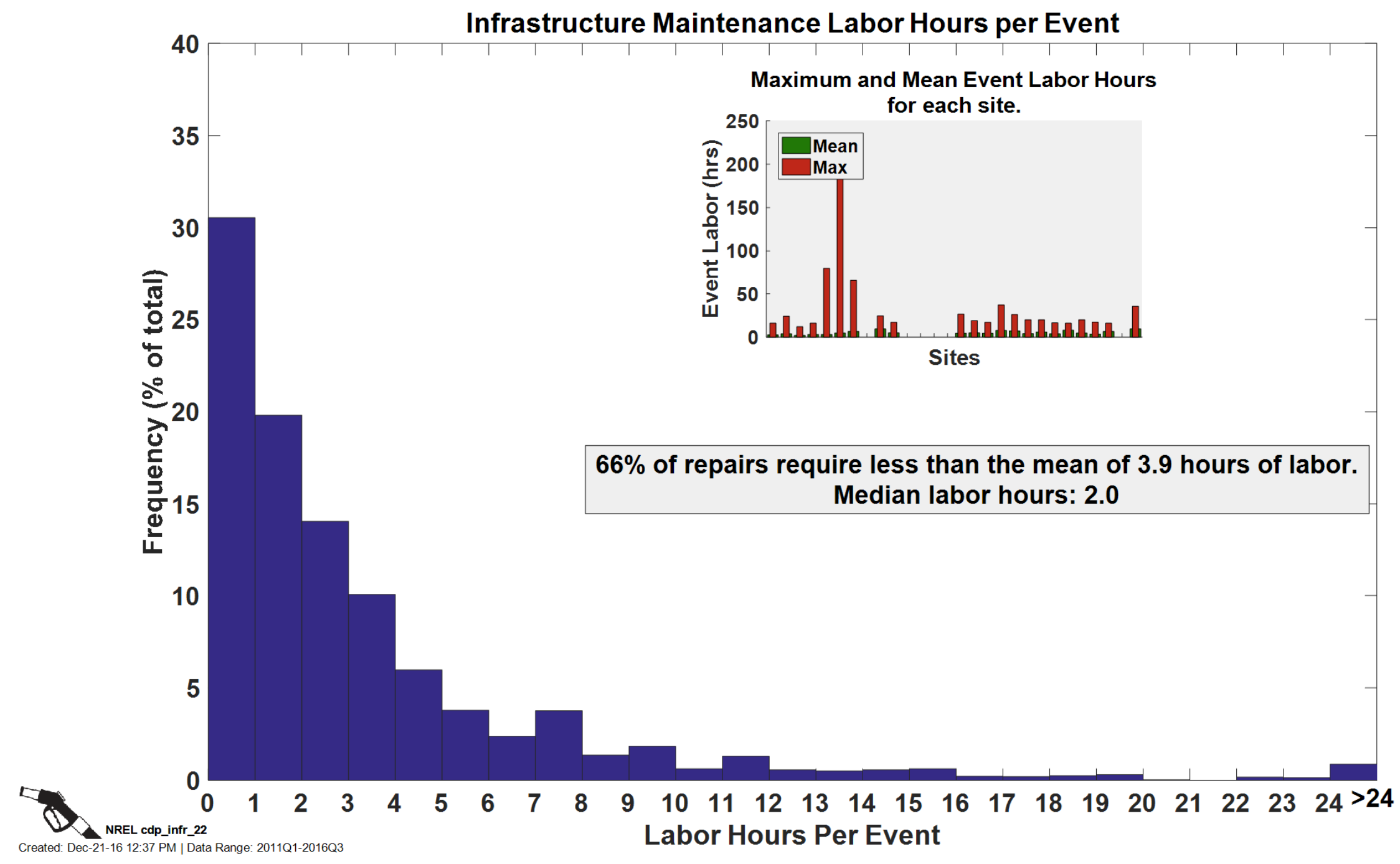




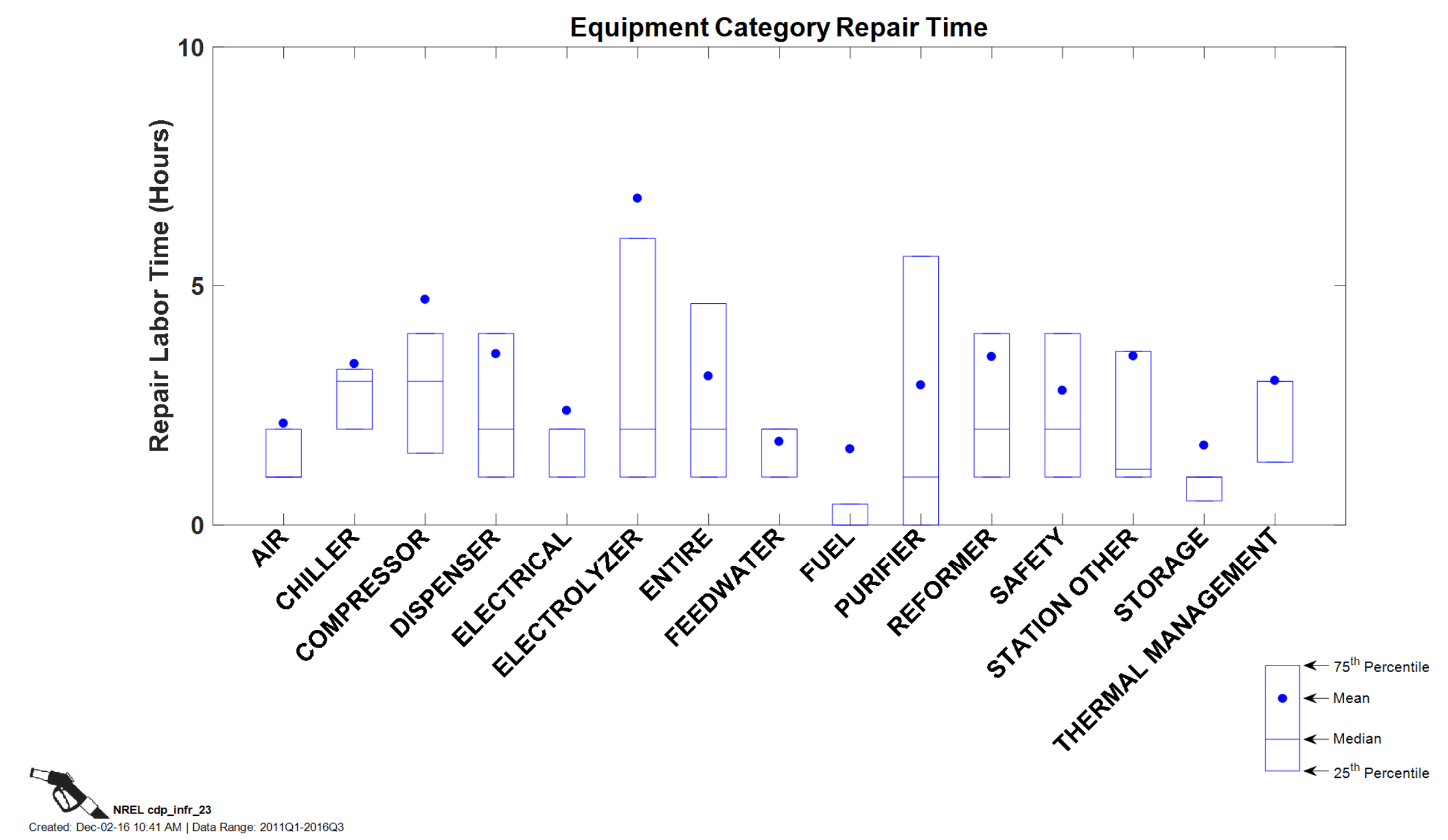


Failure Modes for Top Equipment Categories
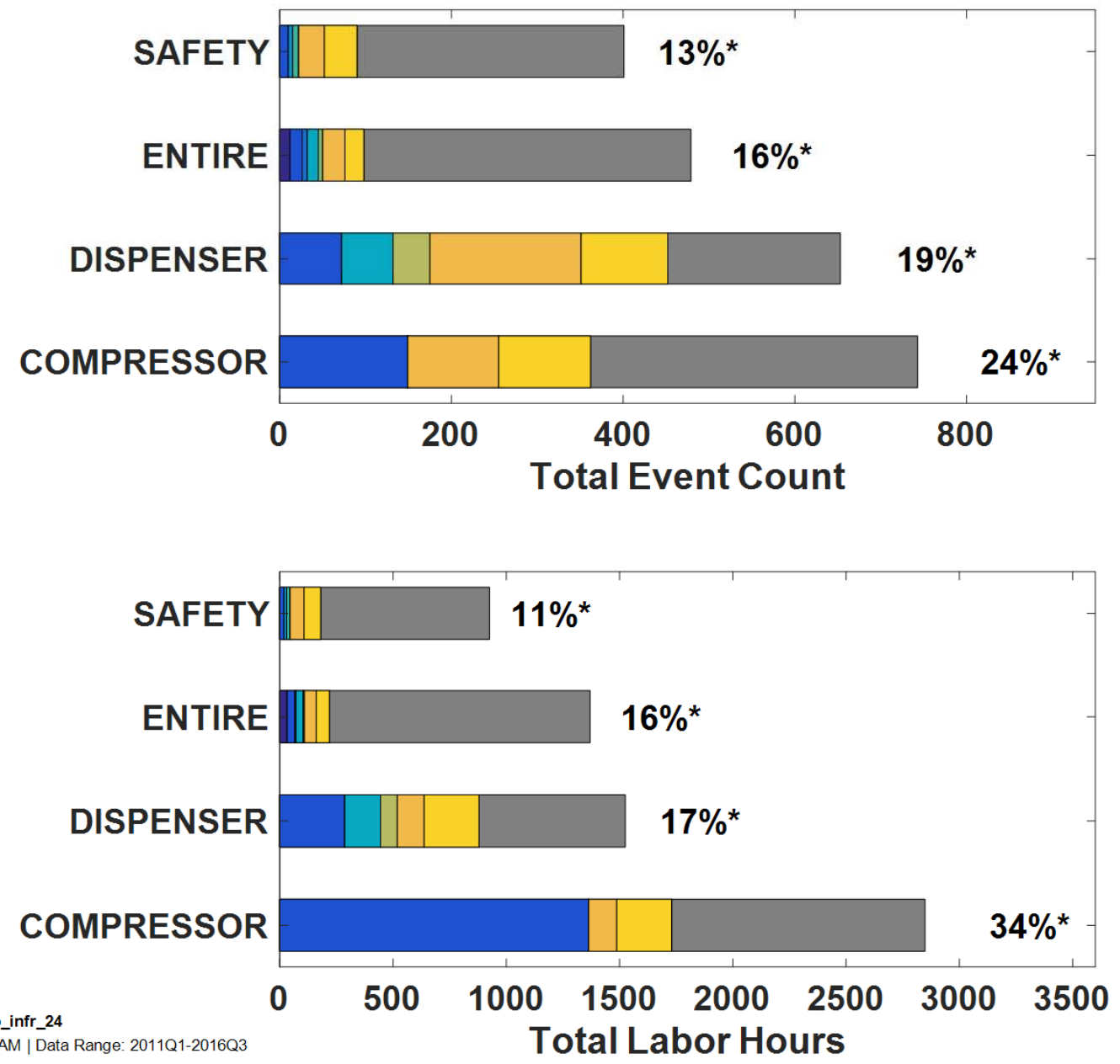

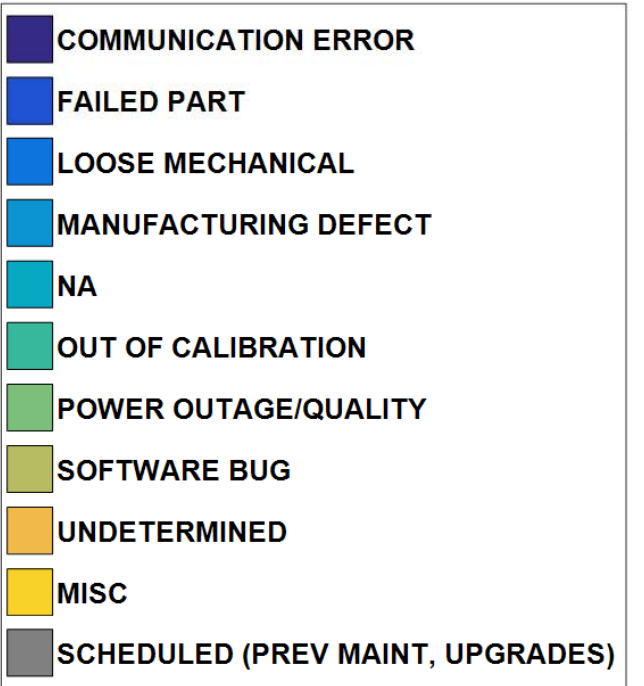

MISC includes the following failure modes: animal damage, collision, communication efrl, contamination, corrective maintenance, debris, design flaw, electrical breaker, ent level low, loose electrical, loose mechanical, maintenance error, manufacturing defect, material deform/degrade/fatigue, moisture, na, operator error, out of calibration, overtemperature, power outage/quality, pressure loss, software bug, stress outside design limit, vibration, other

* Percentage of total events or hours. 

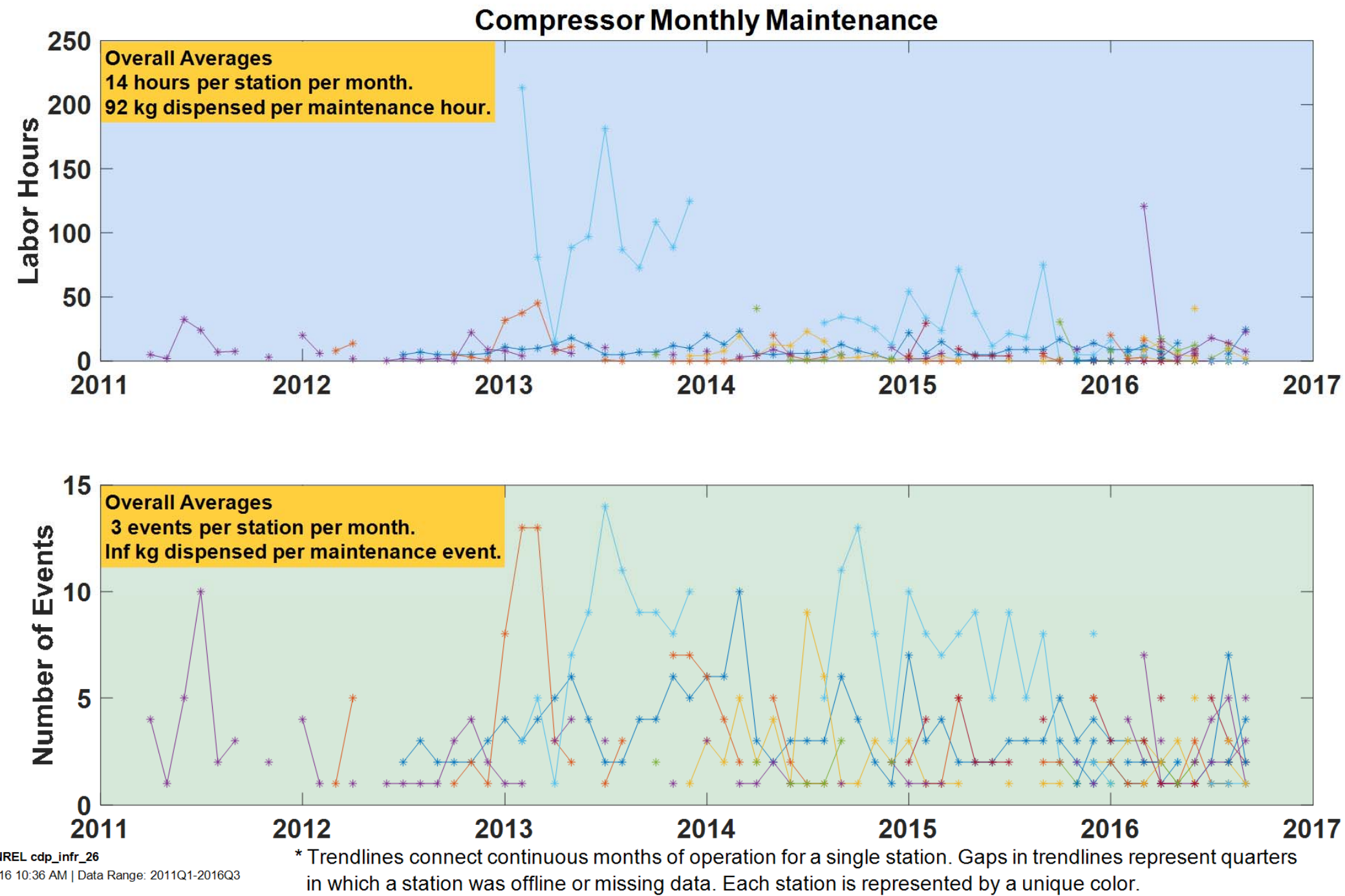


\section{Maintenance Labor Hours by Month}

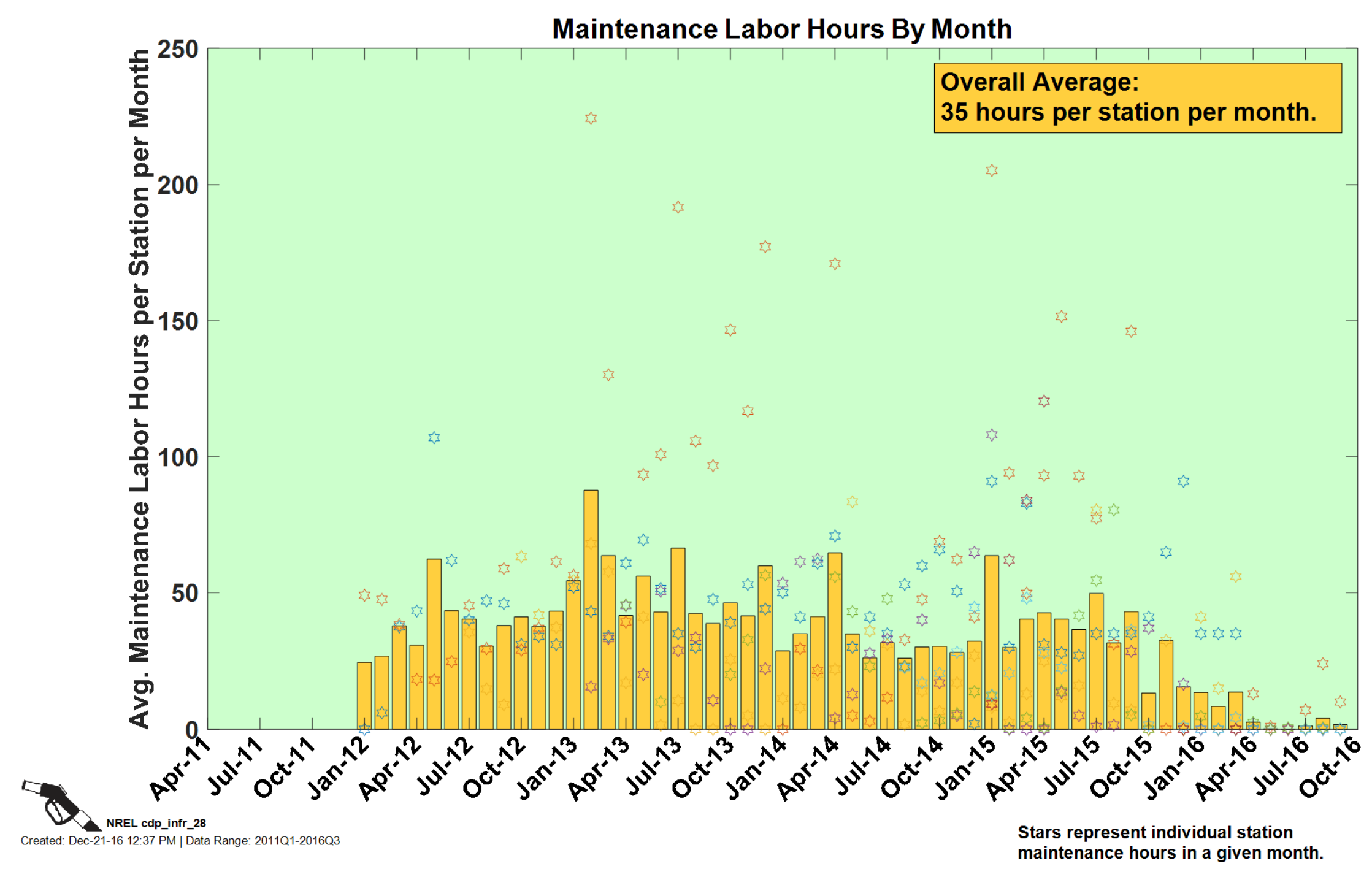




\section{Maintenance Costs Over Time}

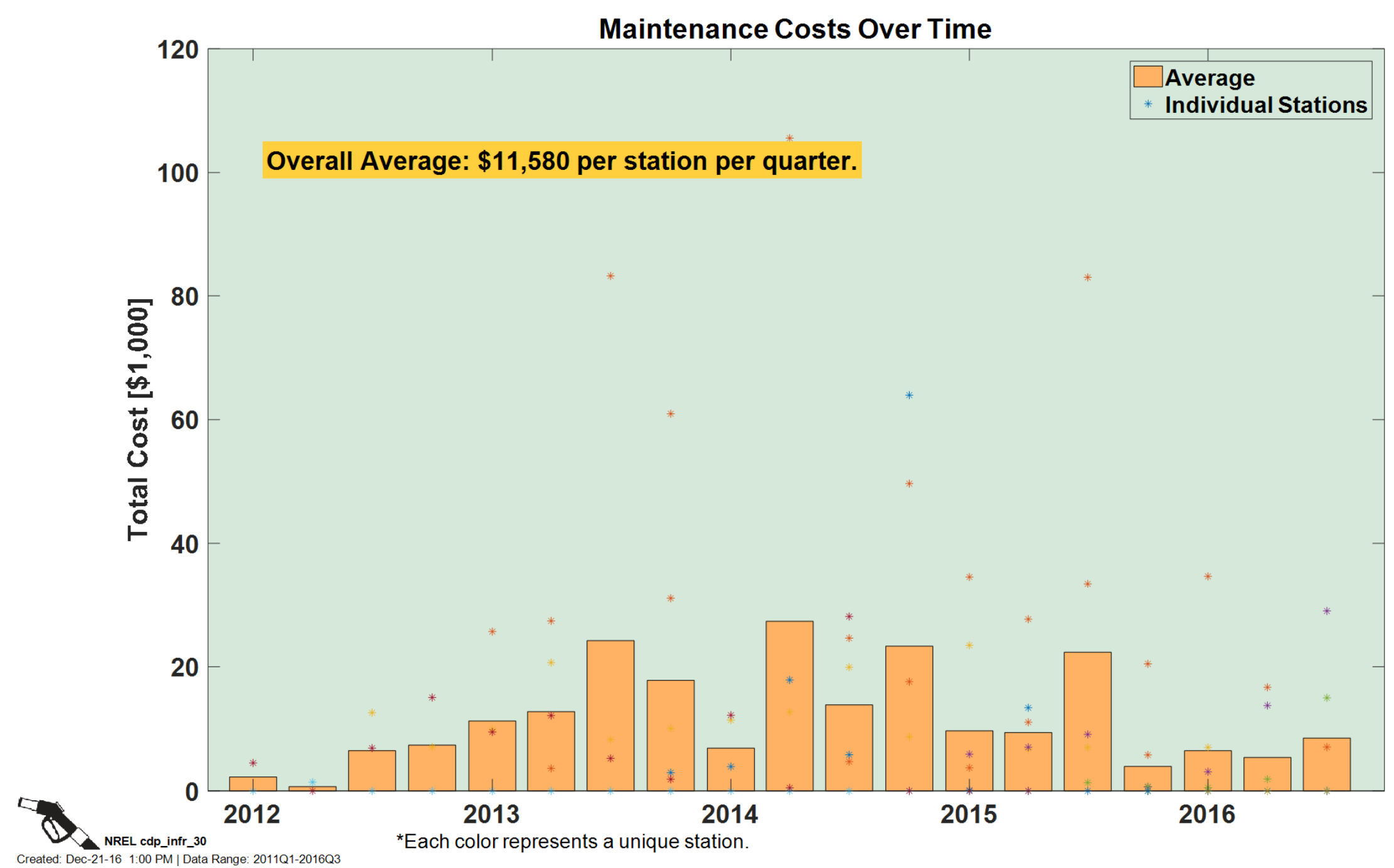




\section{Mean Fills Between Failures}

\section{Mean Fills Between Failures}

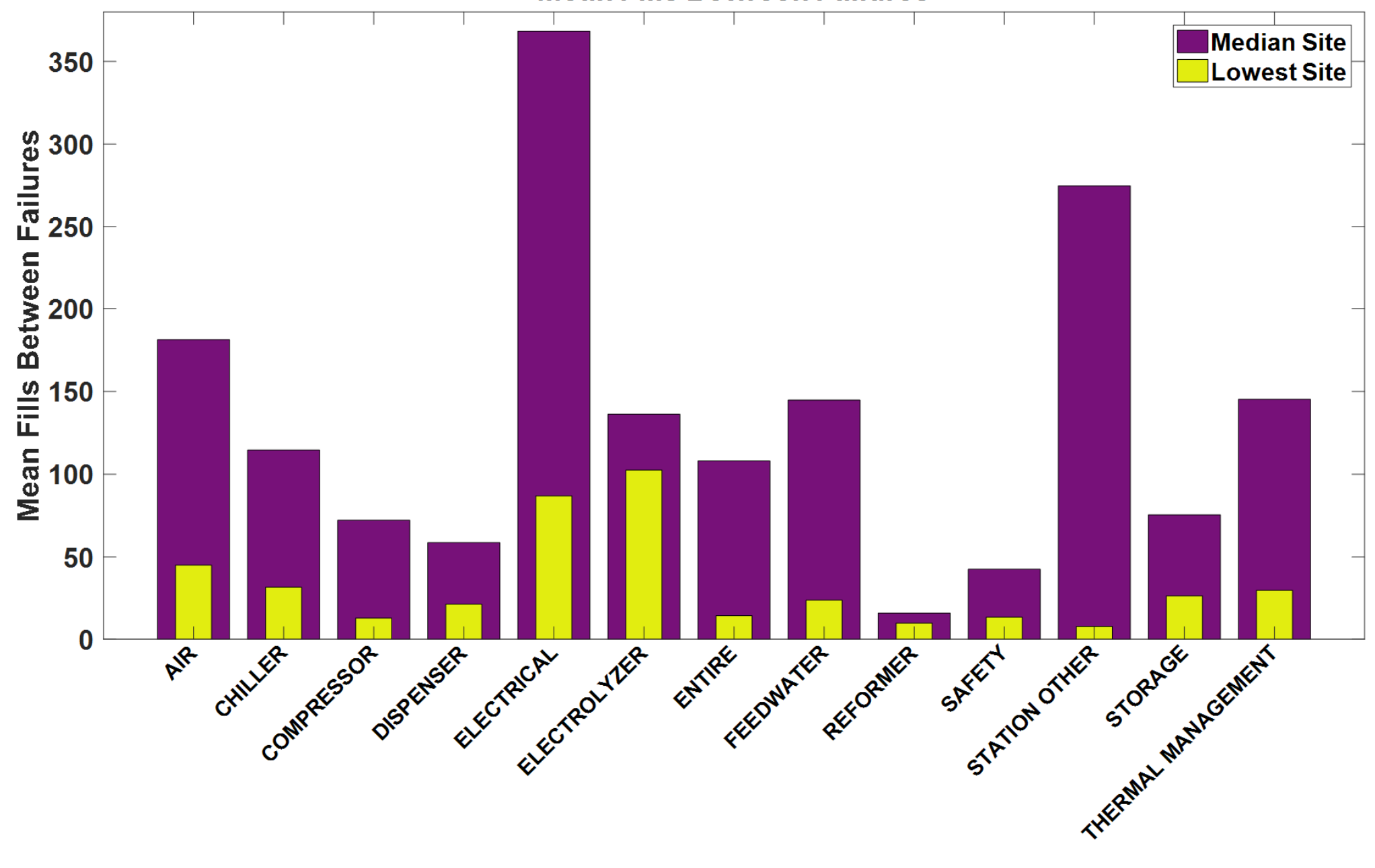

NREL cdp_infr_49

Created: Dec-02-16 10:41 AM | Data Range: 2011Q1-2016Q3 


\section{Reliability Growth by Fills}

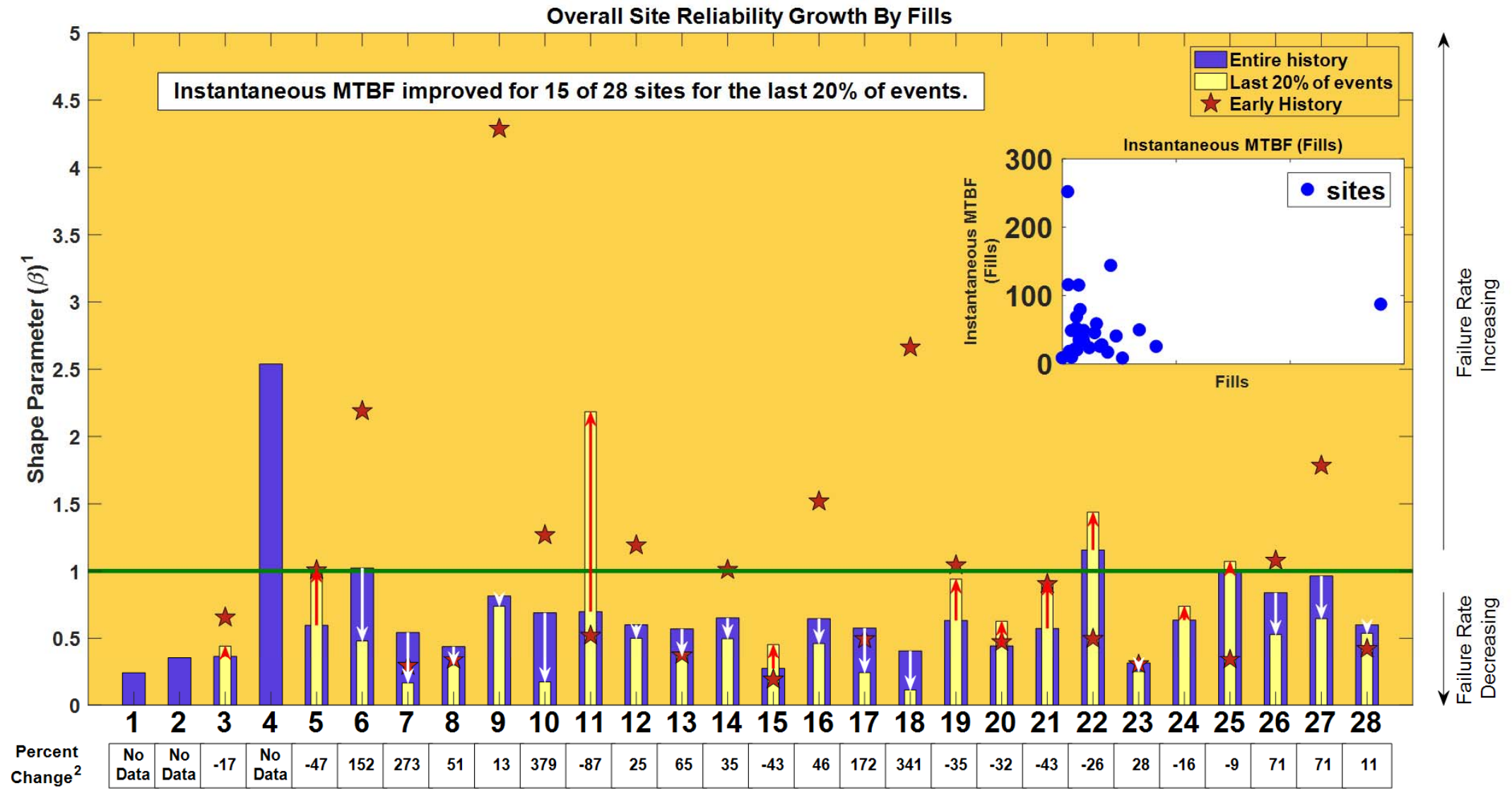

Sites sorted by Increasing Age Fills

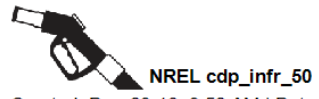

Created: Dec-20-16 9:52 AM | Data Range: 2011Q1-2016Q3
1. IEC 61164:2004(E)., Reliability Growth - Statistical Test and Evaluation Methods, IEC. 2004.

2. \% change in instantaneous mean Fills between failures 


\section{Reliability Growth by Amount Dispensed}

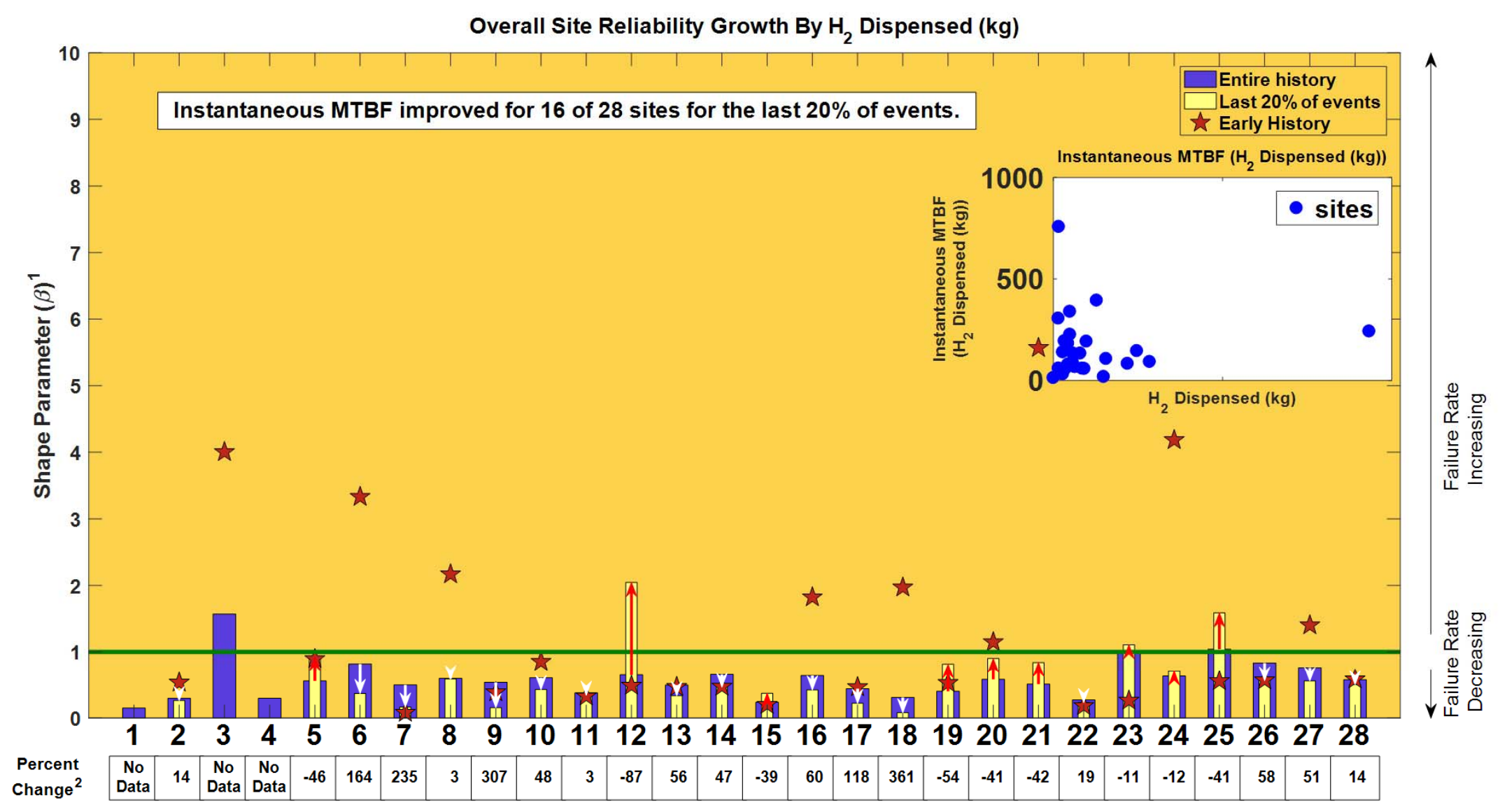

Sites sorted by Increasing Age

$\mathrm{H}_{2}$ Dispensed (kg)

U.

Created: Dec-20-16 9:57 AM | Data Range: 2011Q1-2016Q3
1. IEC 61164:2004(E)., Reliability Growth - Statistical Test and Evaluation Methods, IEC. 2004.

2. \% change in instantaneous mean $\mathrm{H}_{2}$ Dispensed $(\mathrm{kg})$ between failures 


\section{Historical Failure Rate by Fills}

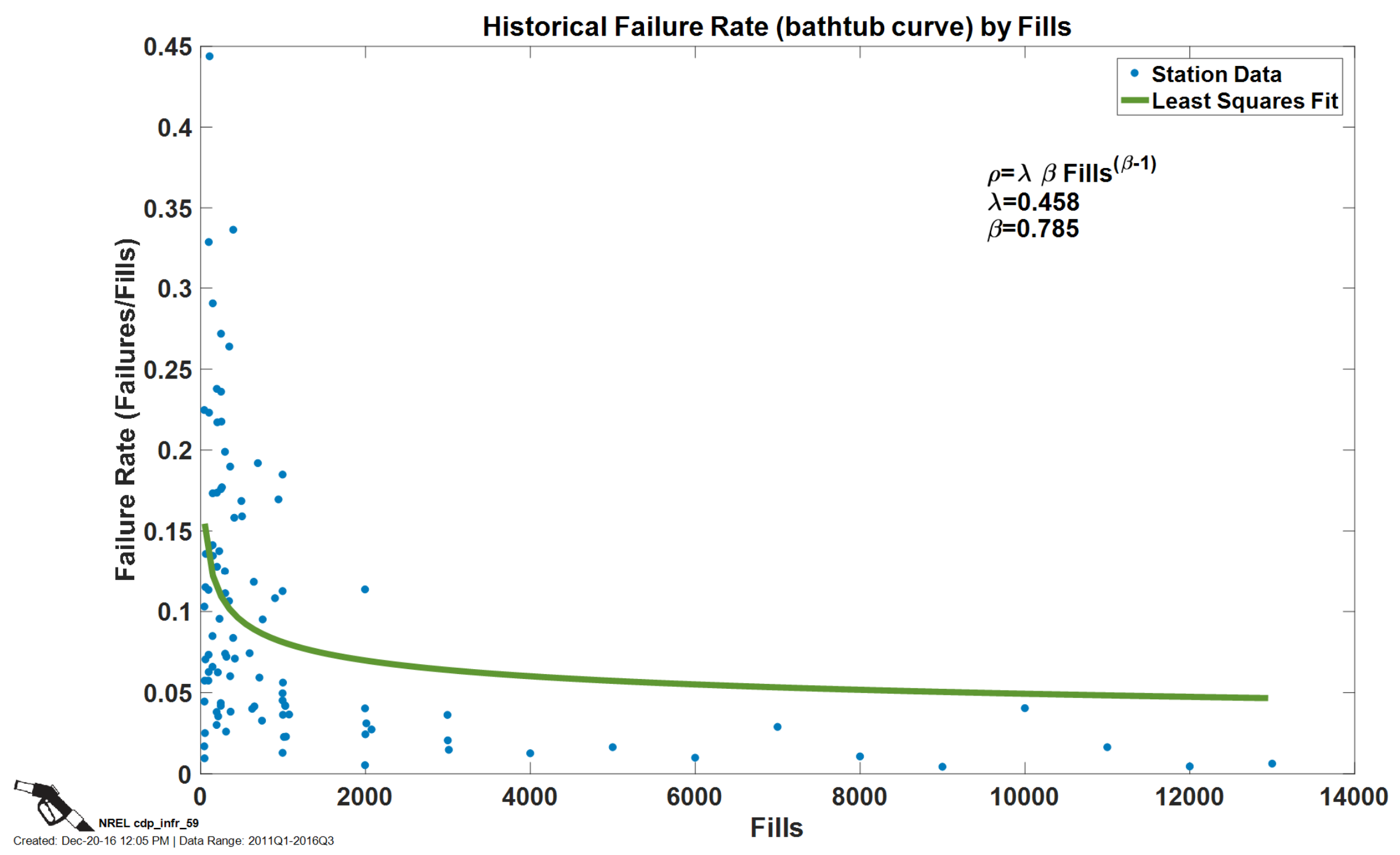




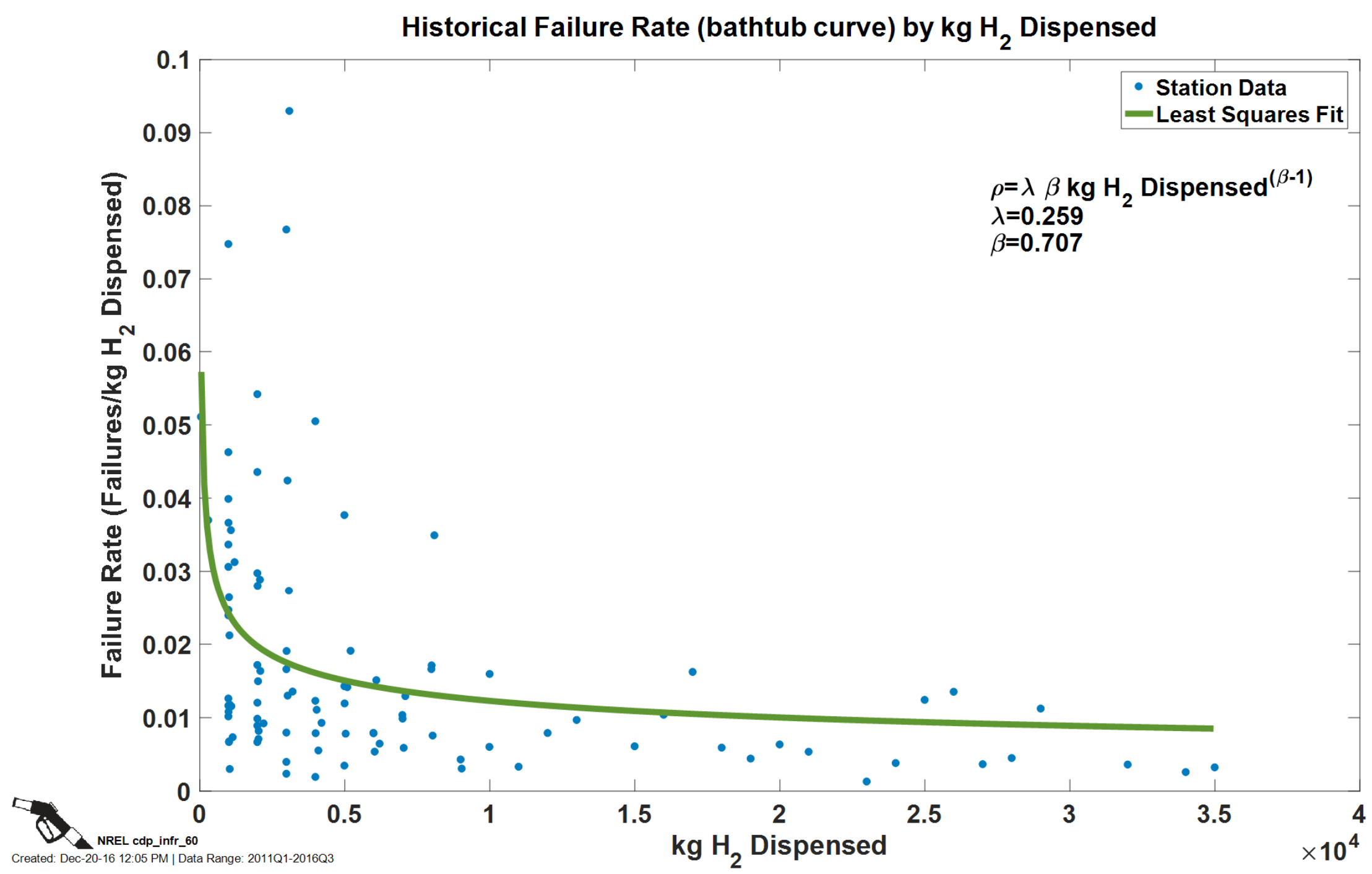




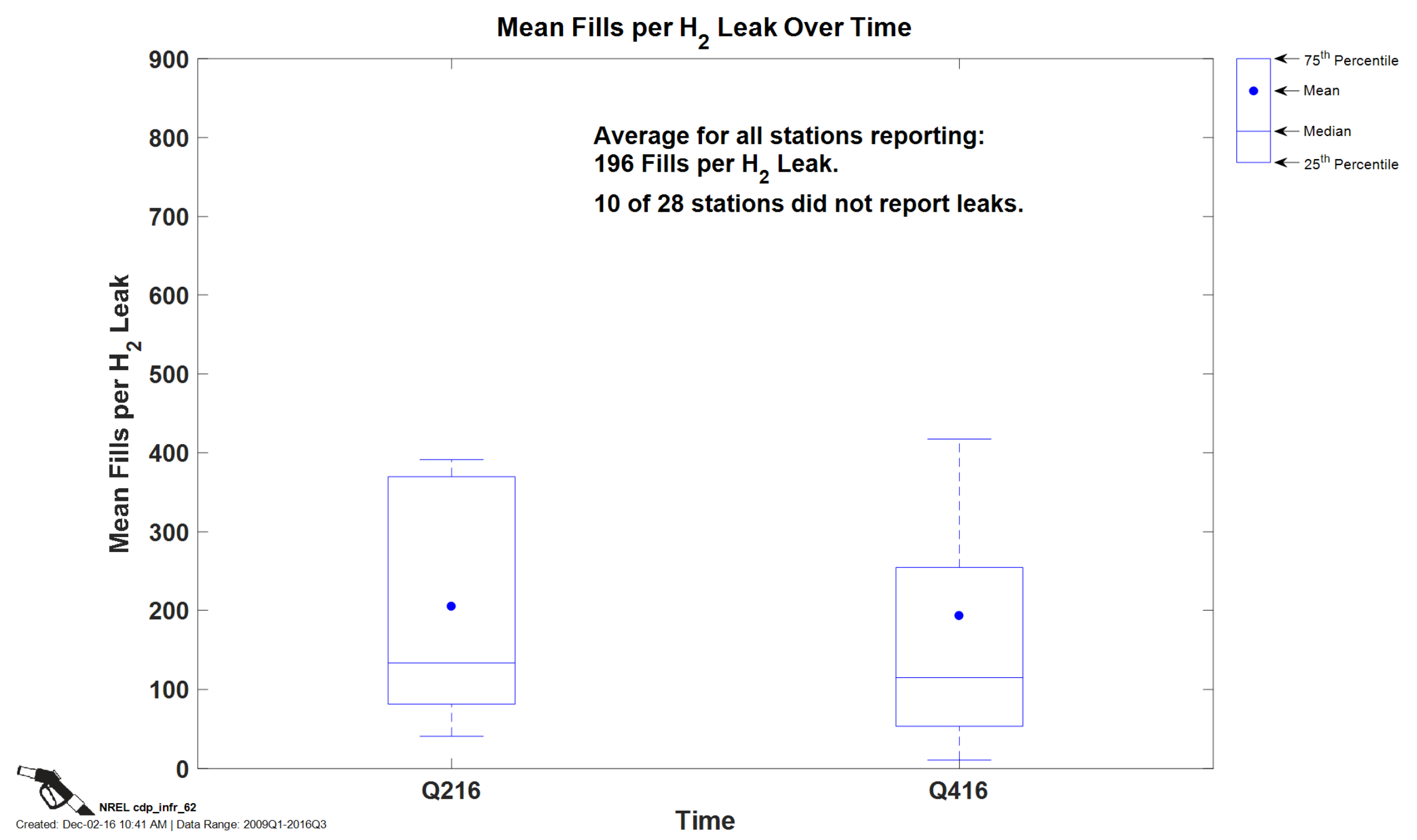




\section{Mean $\mathrm{H}_{2}$ Dispensed Per Leak Over Time}

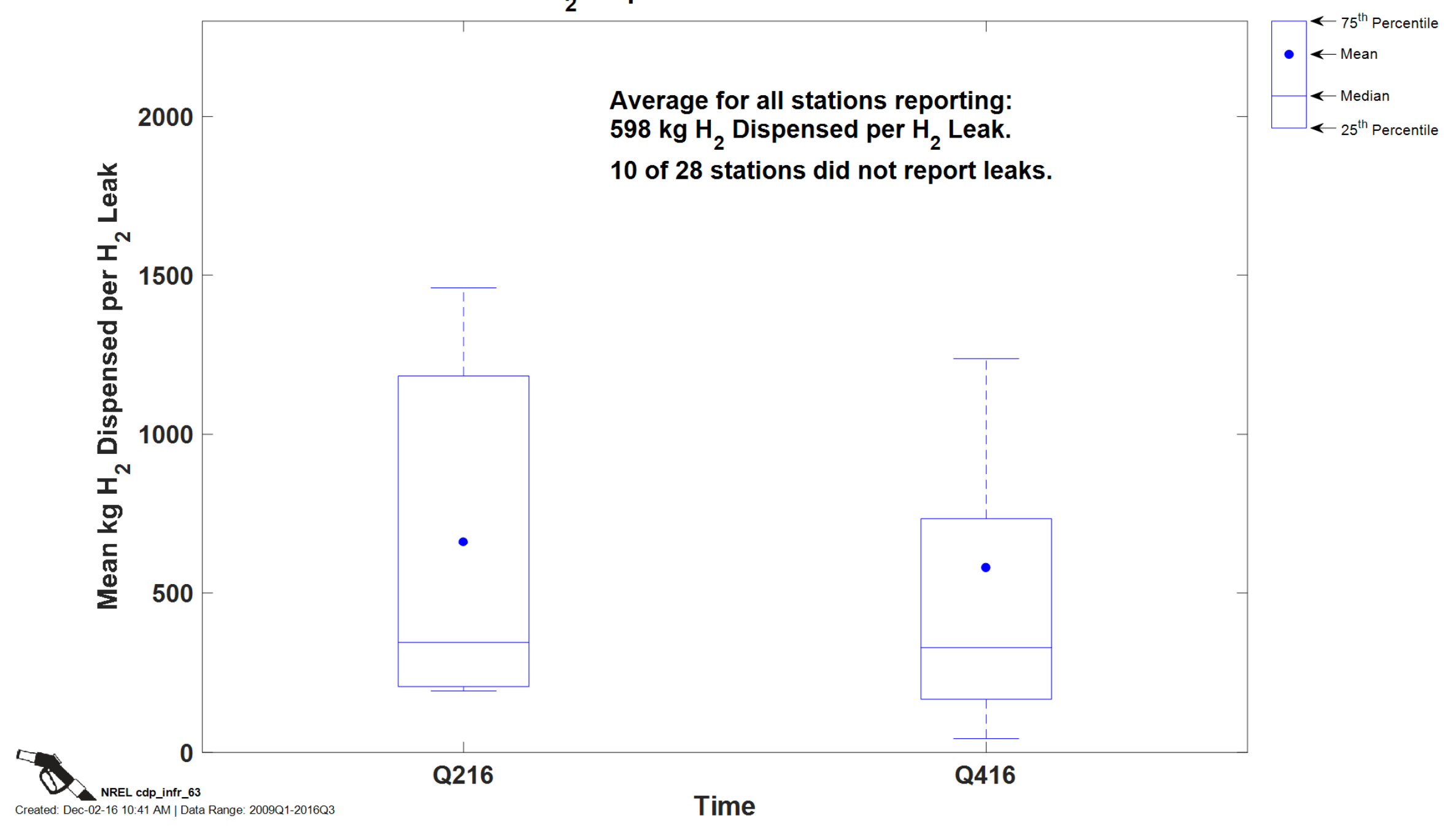


Maintenance Causes and Effects Subsystem: SAFETY

Component: ENTIRE

\section{Component: ENTIRE}

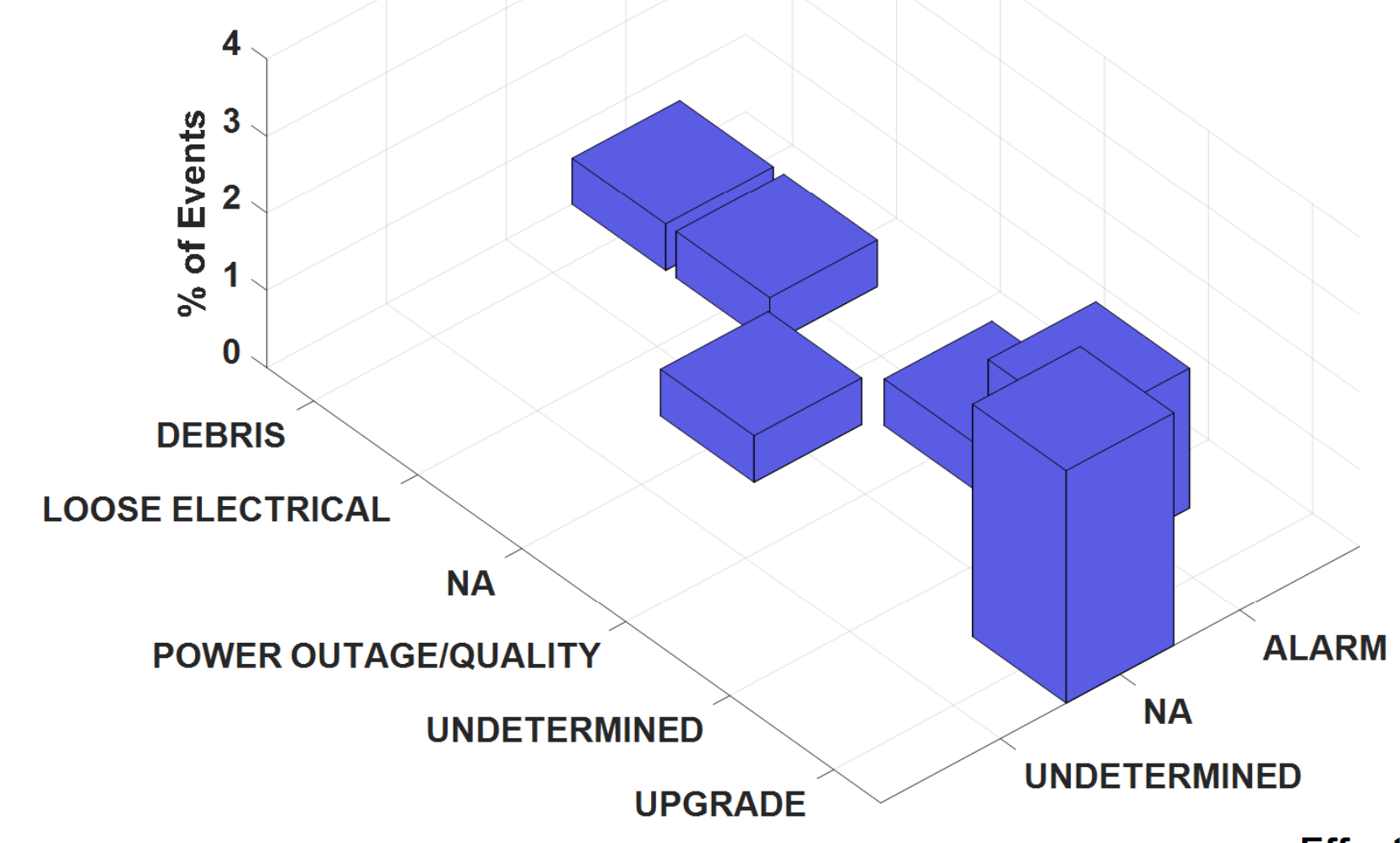

DEBRIS

LOOSE ELECTRICAL

Causes 


\section{Maintenance Causes and Effects \\ Subsystem: SAFETY \\ Component: SENSOR}

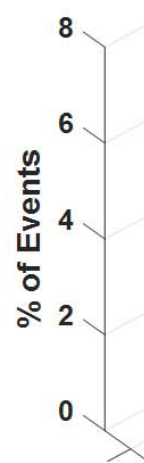

ENVIRONMENTAL FACTORS

FAILED PART LOOSE ELECTRICAL MAINTENANCE ERROR MANUFACTURING DEFECT MOISTURE OUT OF CALIBRATION POWER OUTAGE/QUALITY UNDETERMINED UPGRADE ANIMAL DAMAGE COLLISION

\section{Causes} INSTALLATION ERROR
Preventative Maintenance accounted for $53 \%$ of all events. Suppressed in the plot to show detail for other causes.

\section{(1) NREL cdp_infr_65}


Maintenance Causes and Effects Subsystem: SAFETY

Component: SOFTWARE

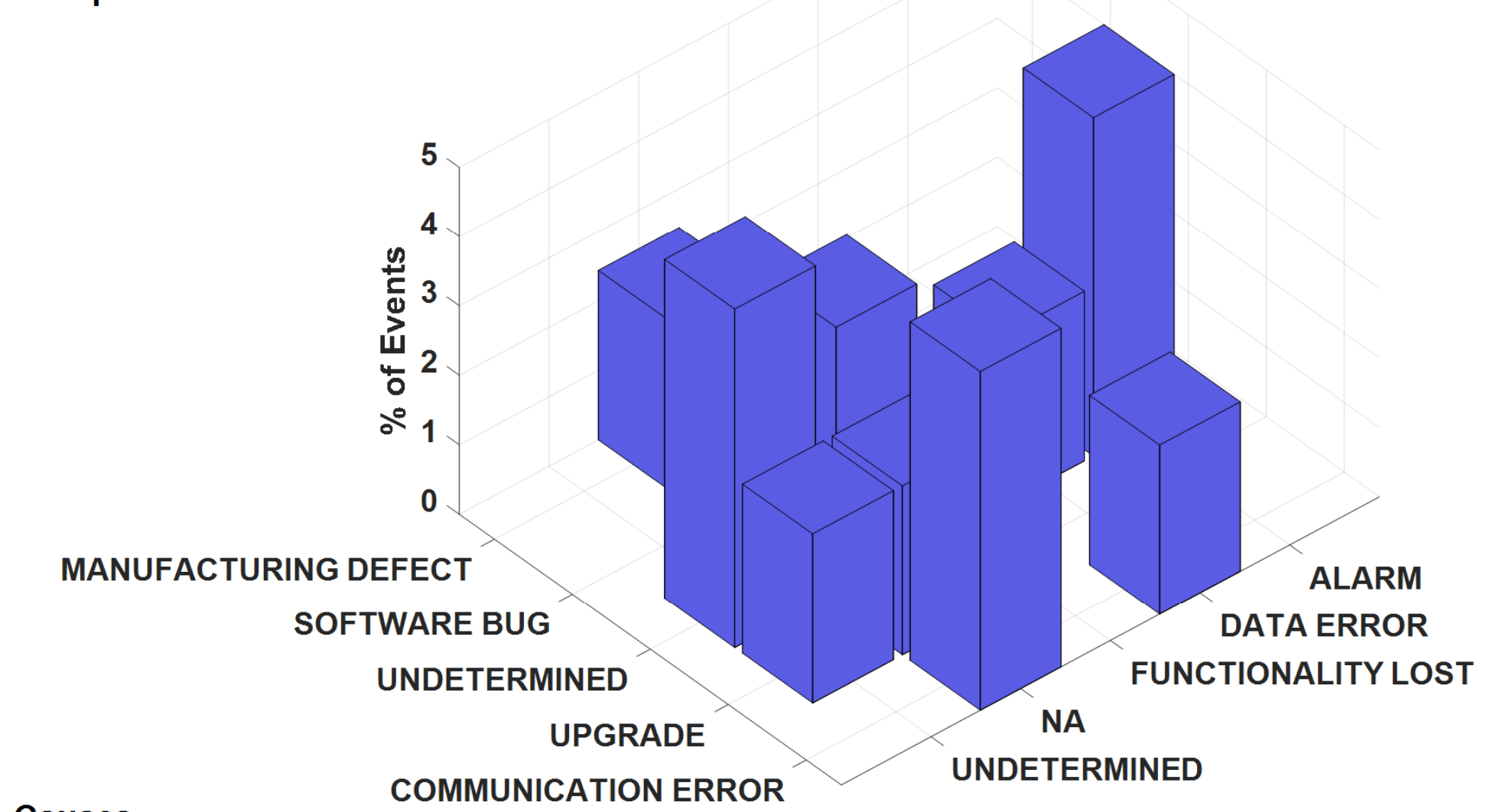

Causes

COMMUNICATION ERROR

Effects 


\section{Maintenance Causes and Effects Subsystem: DISPENSER Component: ENTIRE}

.
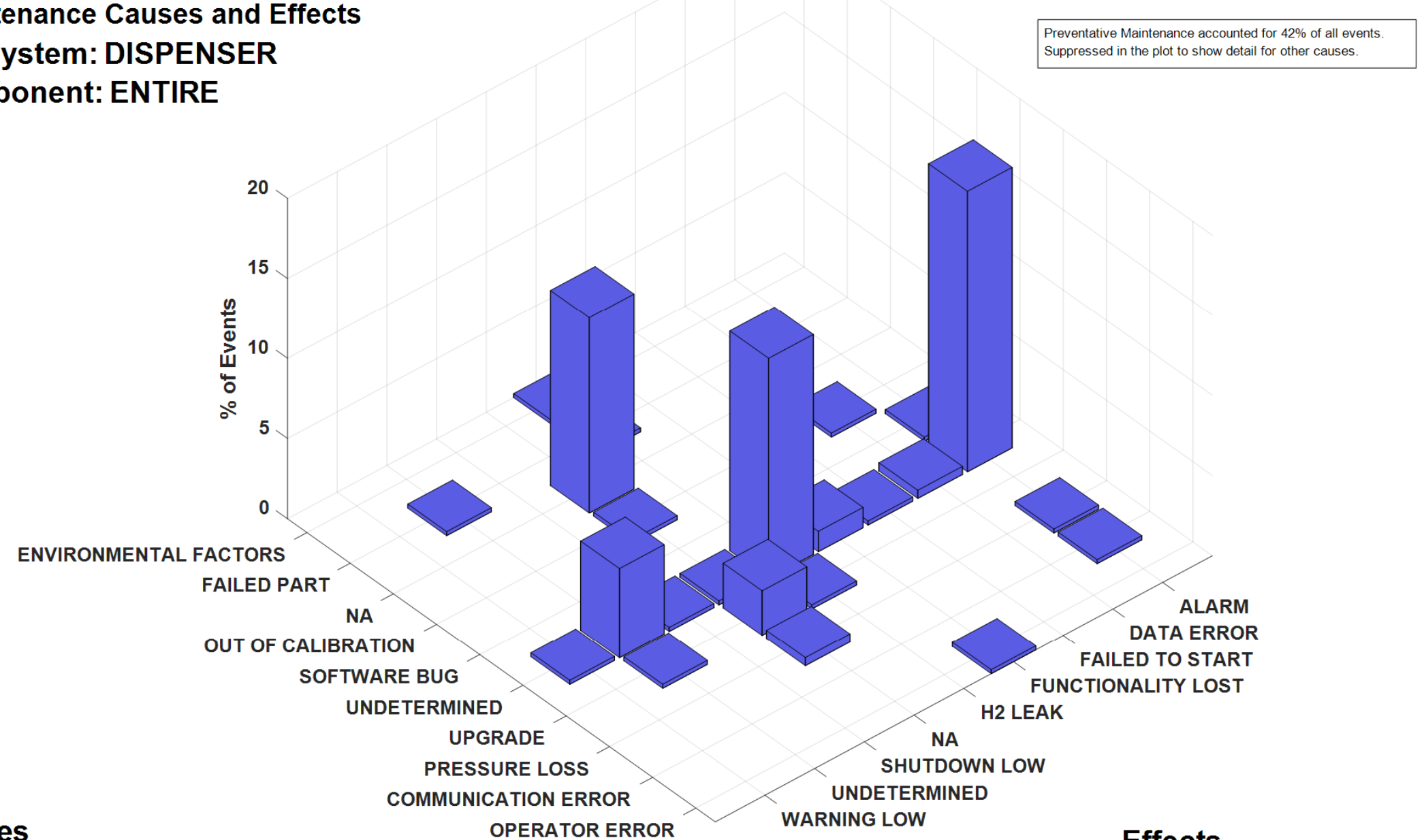

\section{Causes}

OPERATOR ERROR

\section{Effects}


Maintenance Causes and Effects Subsystem: DISPENSER

Component: VALVE

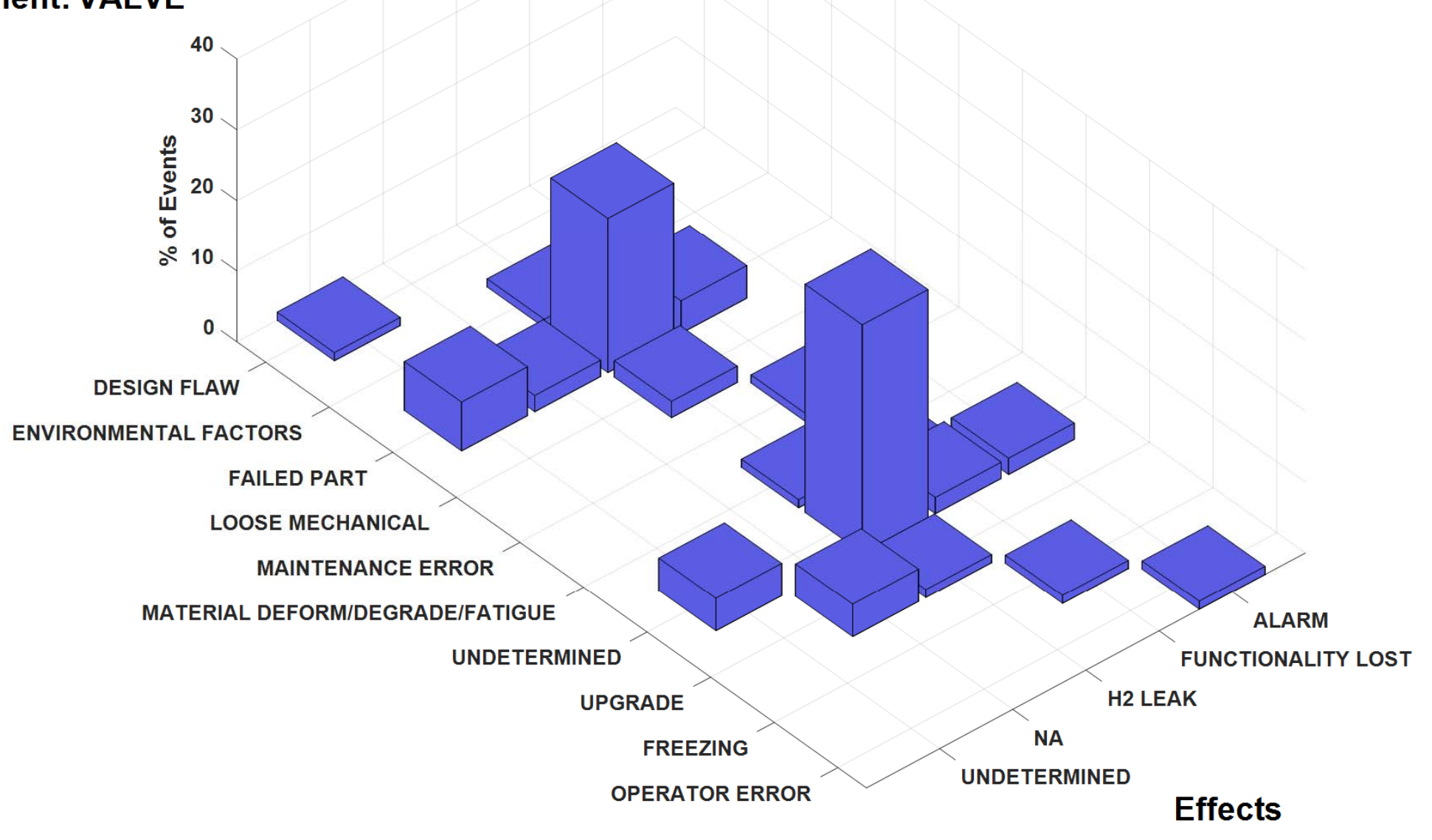

Causes

OPERATOR ERROR

Effects 
Maintenance Causes and Effects Subsystem: DISPENSER

Component: SOFTWARE

\section{COMPONent: SOFTWARE}

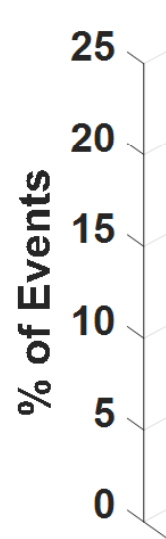

FAILED PART

OUT OF CALIBRATION

SOFTWARE BUG

UNDETERMINED

UPGRADE

COMMUNICATION ERROR

Causes

INSTALLATION ERROR

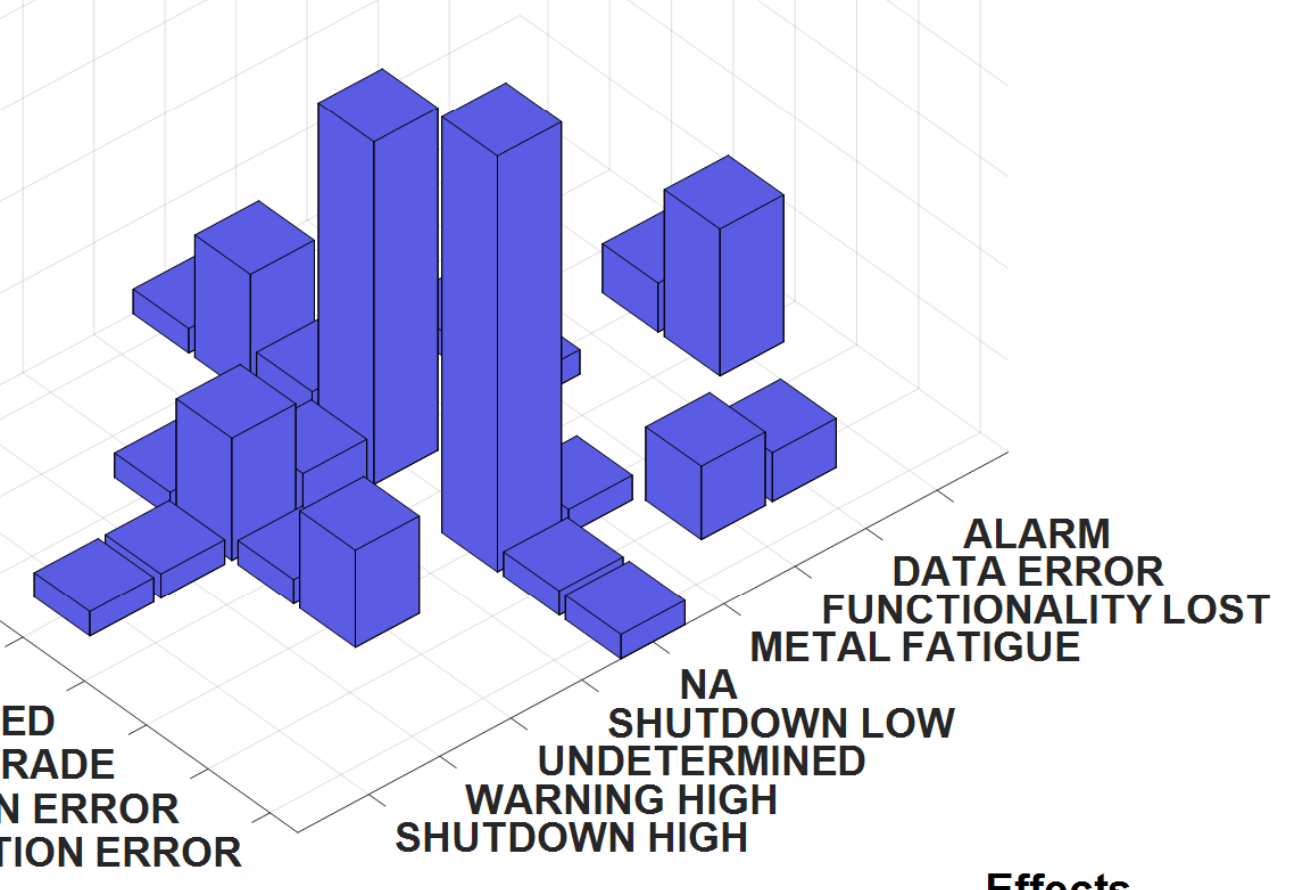

Effects 


\section{Maintenance Causes and Effects Subsystem: COMPRESSOR Component: ENTIRE}

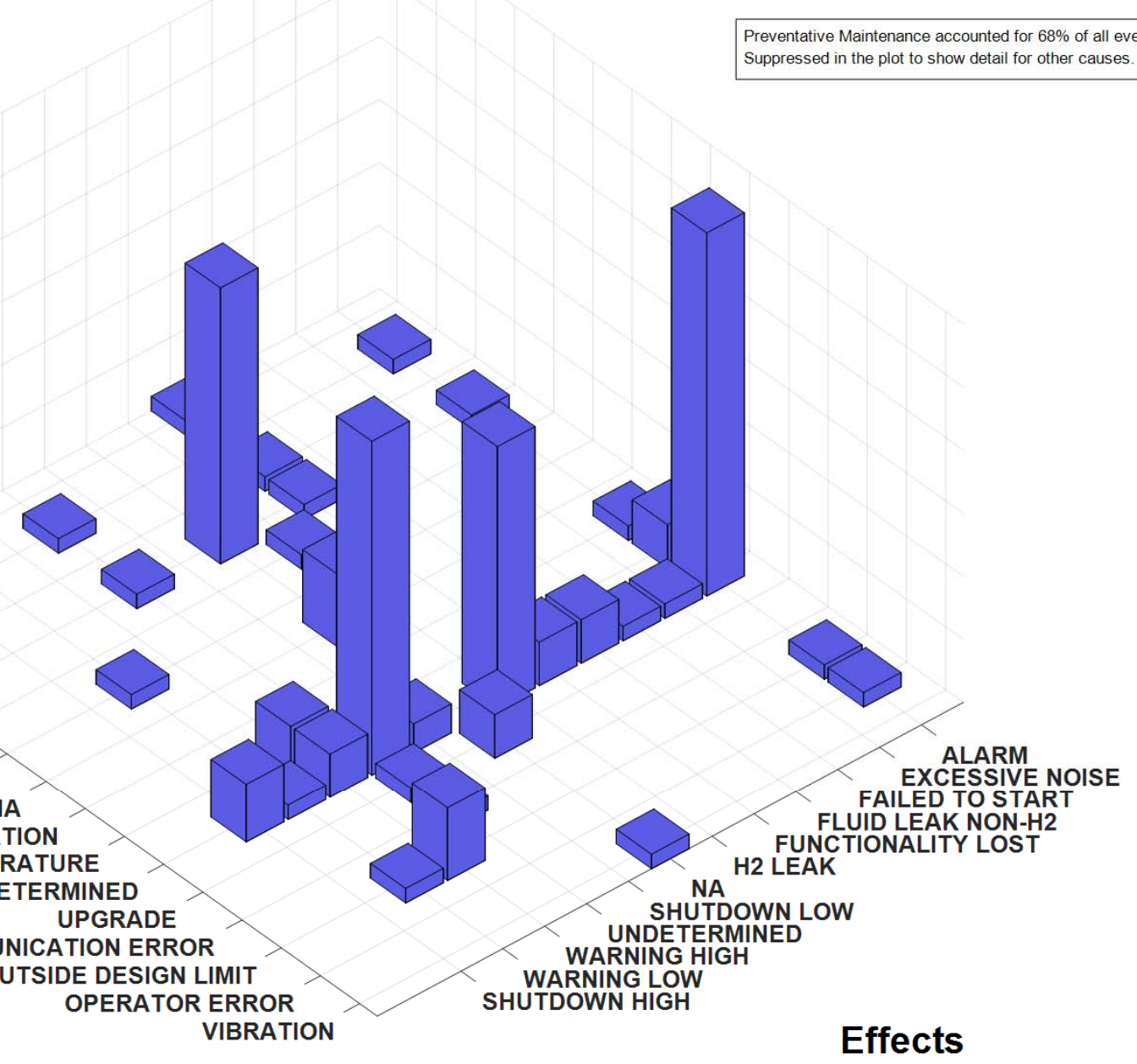

\section{Causes}

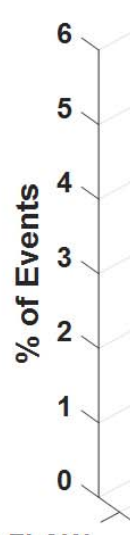

DESIGN FLAW ELECTRICAL BREAKER ENVIRONMENTAL FACTORS

FAILED PART LOOSE MECHANICAL MAINTENANCE ERROR

$$
\begin{aligned}
& \text { OUT OF CALIBRATION } \\
& \text { OVERTEMPERATURE }
\end{aligned}
$$

$$
\text { UNDETERMINED }
$$

COMMUNICATION ERROR STRESS OUTSIDE DESIGN LIMIT VIBRATION

\section{Effects}


Maintenance Causes and Effects Subsystem: COMPRESSOR Component: VALVE

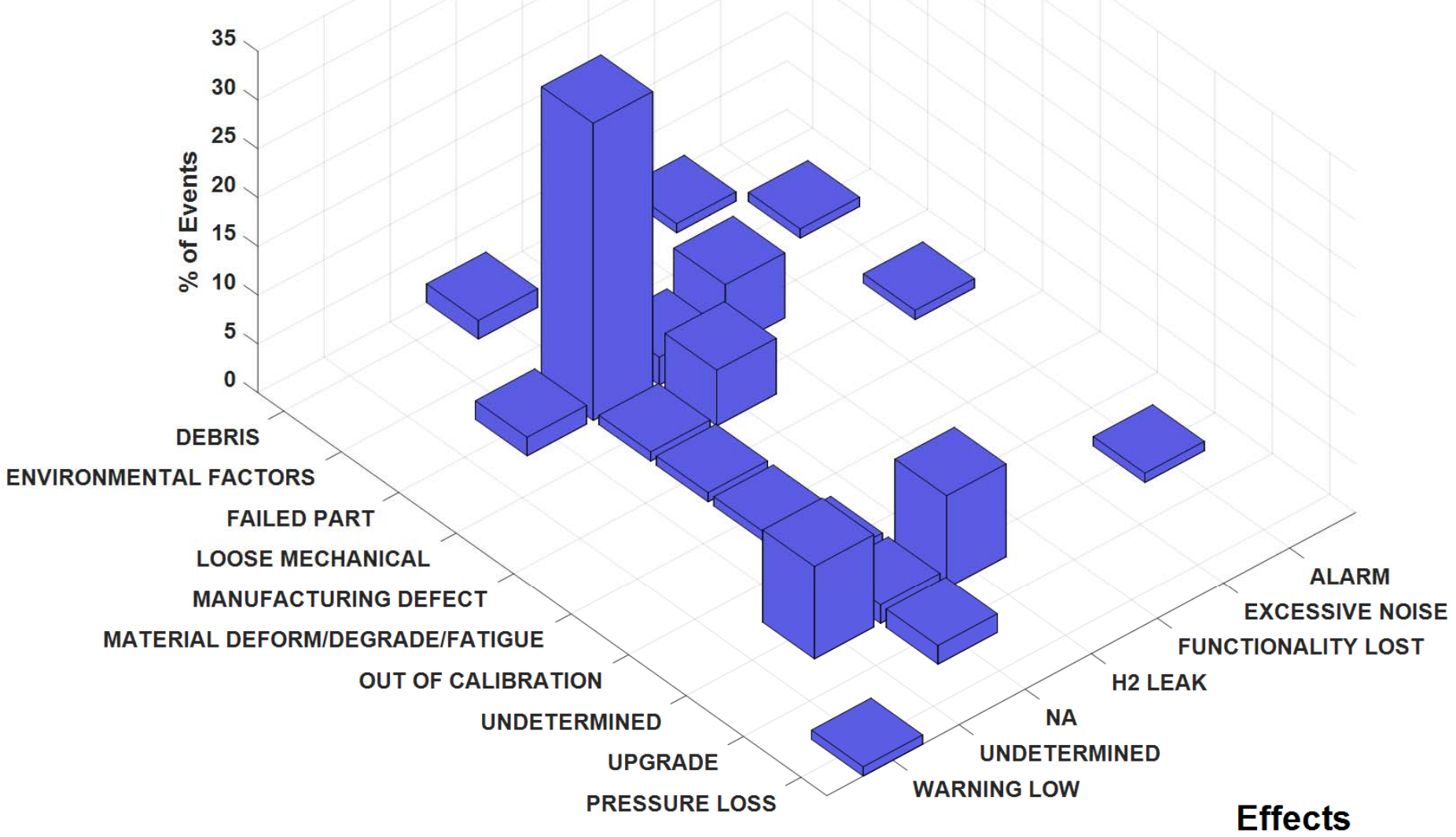

\section{Causes} PRESSURE LOSS

Effects 


\section{Maintenance Causes and Effects Subsystem: COMPRESSOR Component: SEAL}

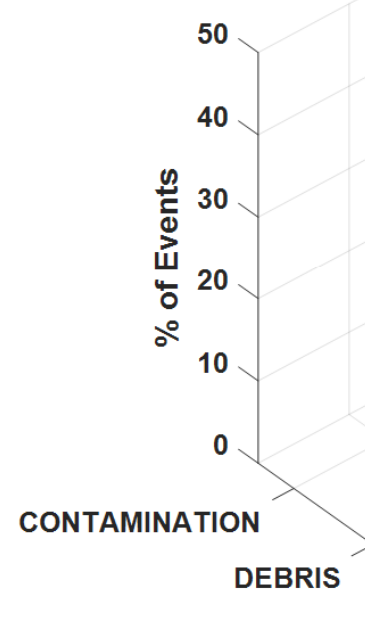

ENVIRONMENTAL FACTORS

FAILED PART

MANUFACTURING DEFECT

MATERIAL DEFORM/DEGRADE/FATIGUE

UNDETERMINED

UPGRADE

INSTALLATION ERROR

Causes

Corrective Maintenance 
Performance 
Hydrogen Dispensed By Quarter

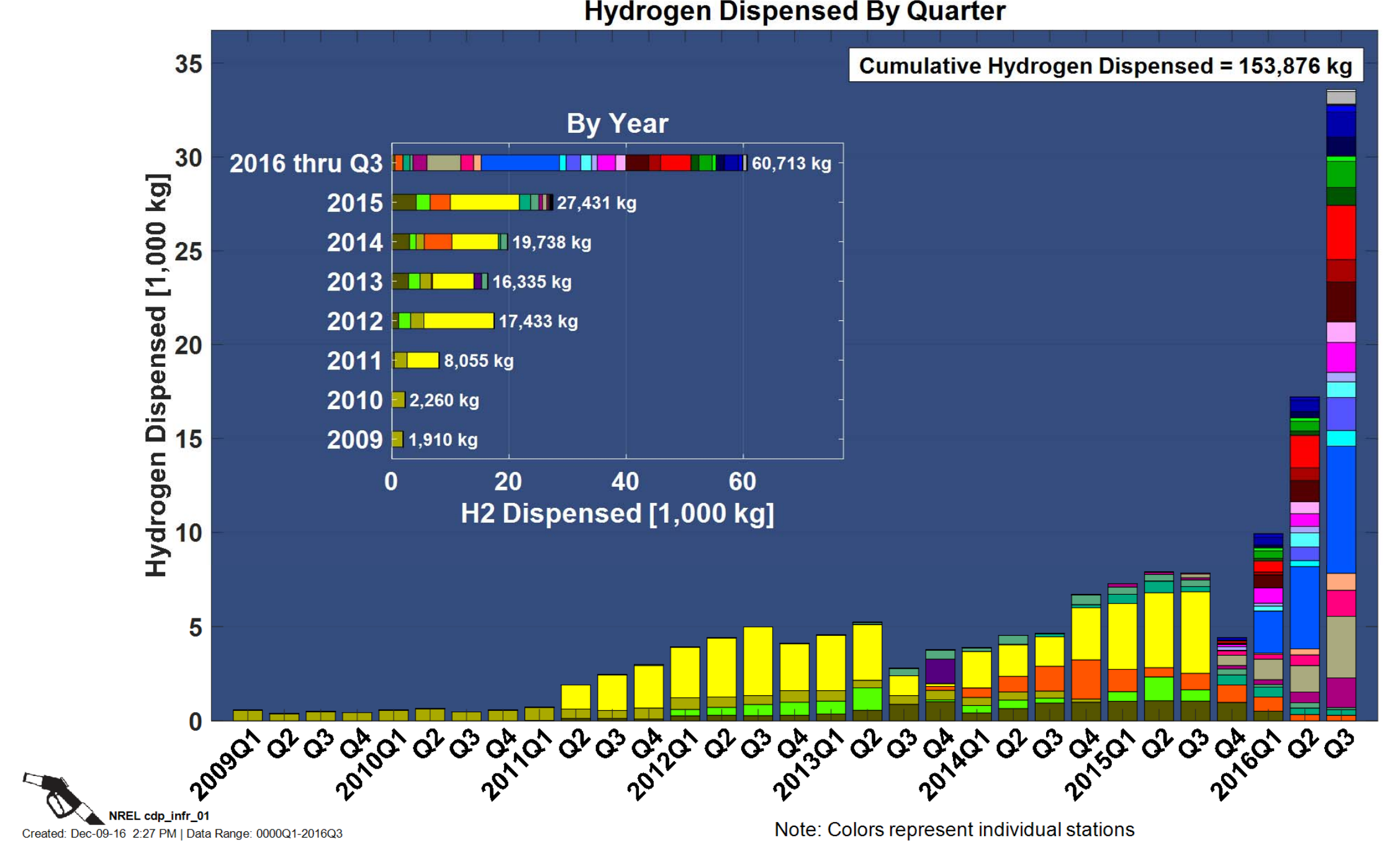




\section{Hydrogen Fills By Quarter}

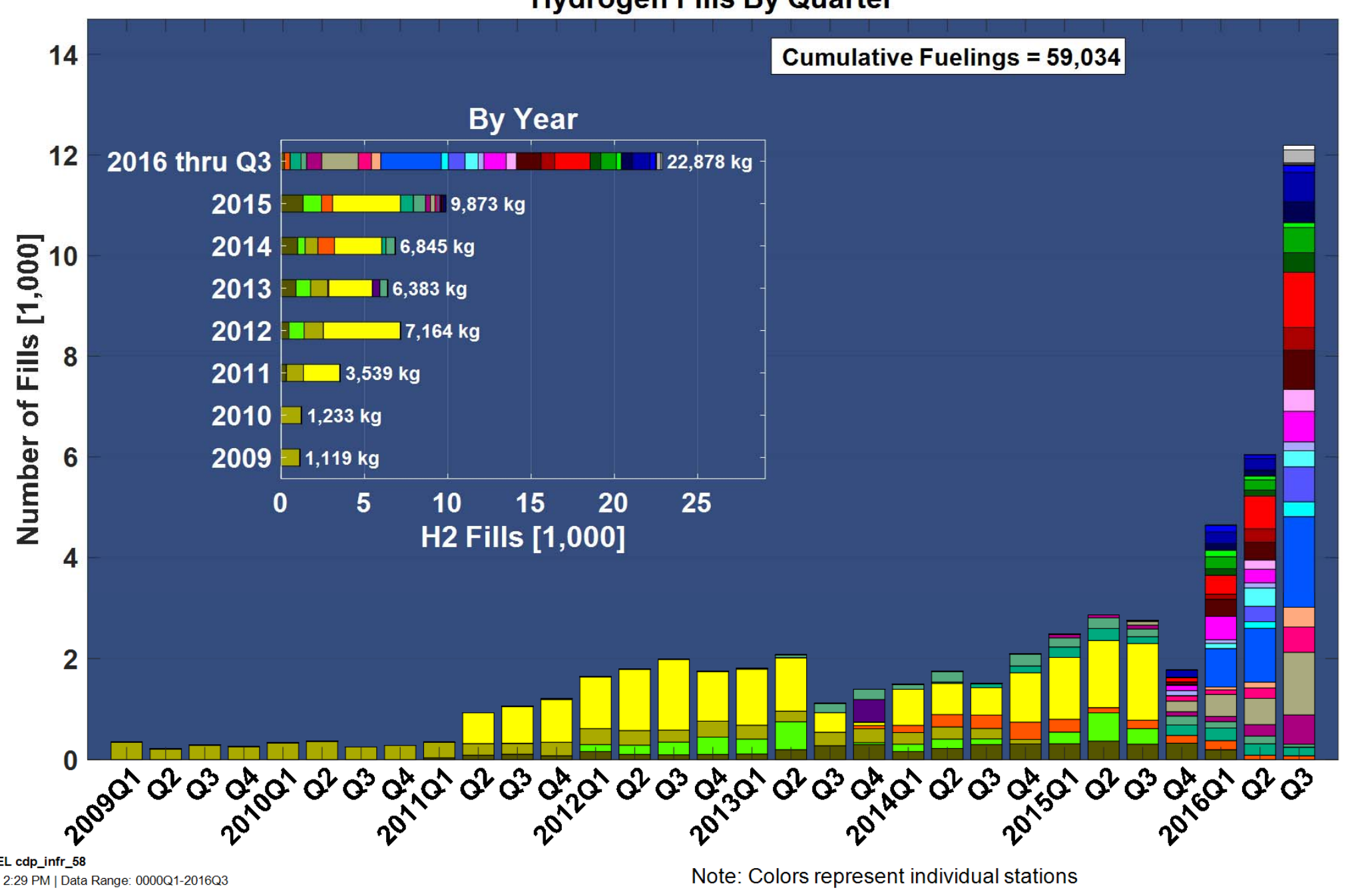




\section{Histogram of Fueling Rates}

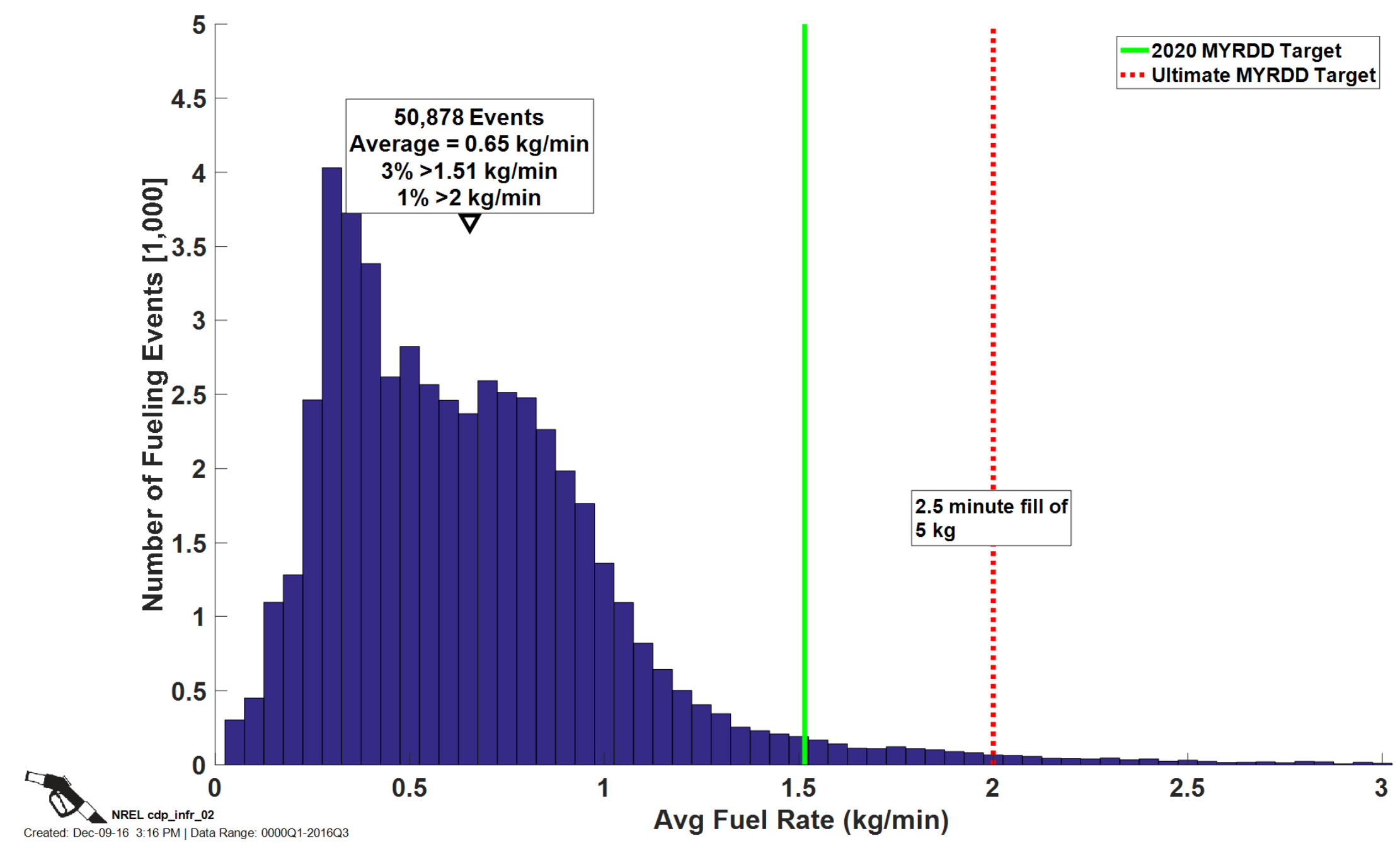




\section{Histogram of Fueling Times}

\section{Histogram of Fueling Times}

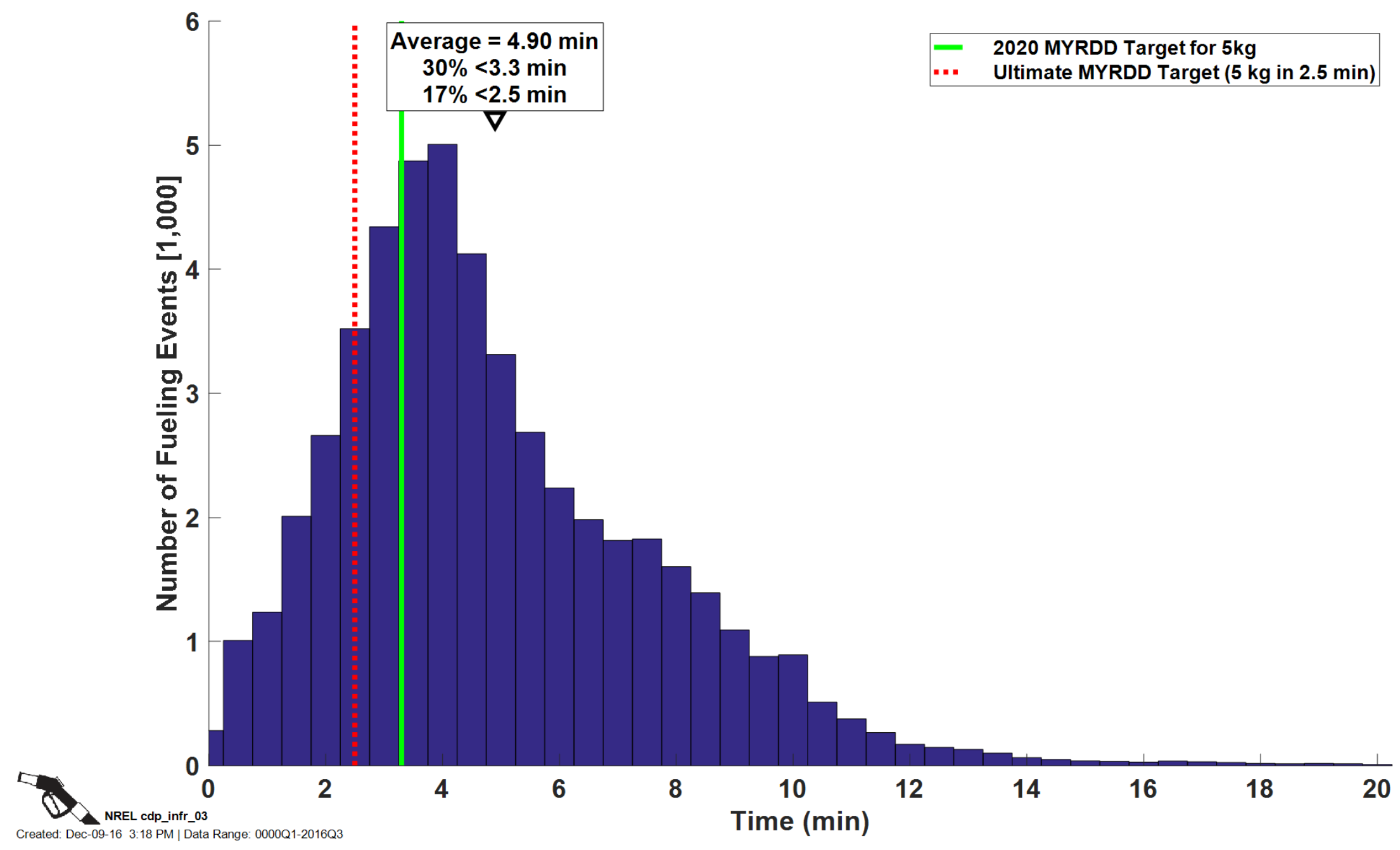




\section{Histogram of Fueling Amounts}

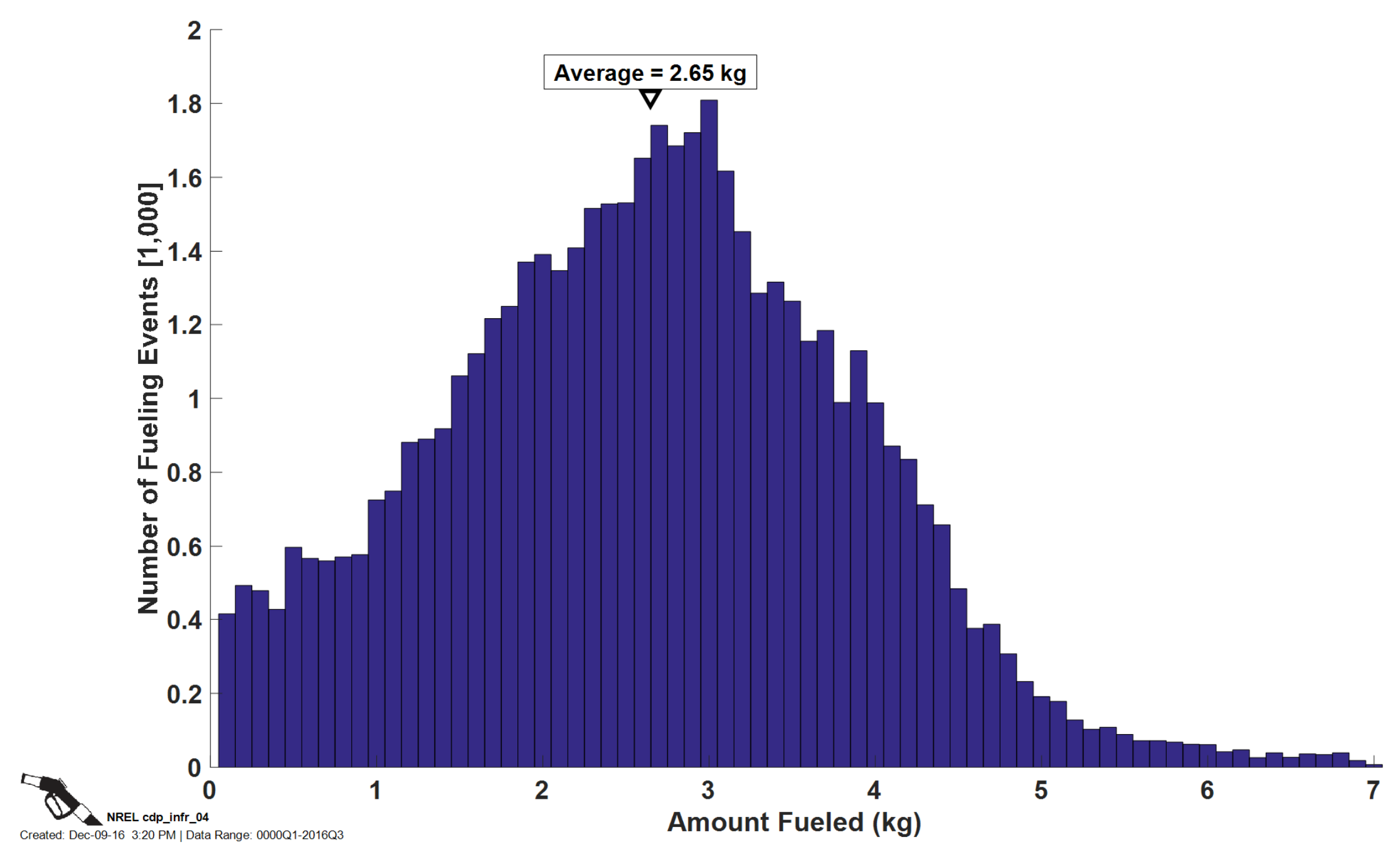



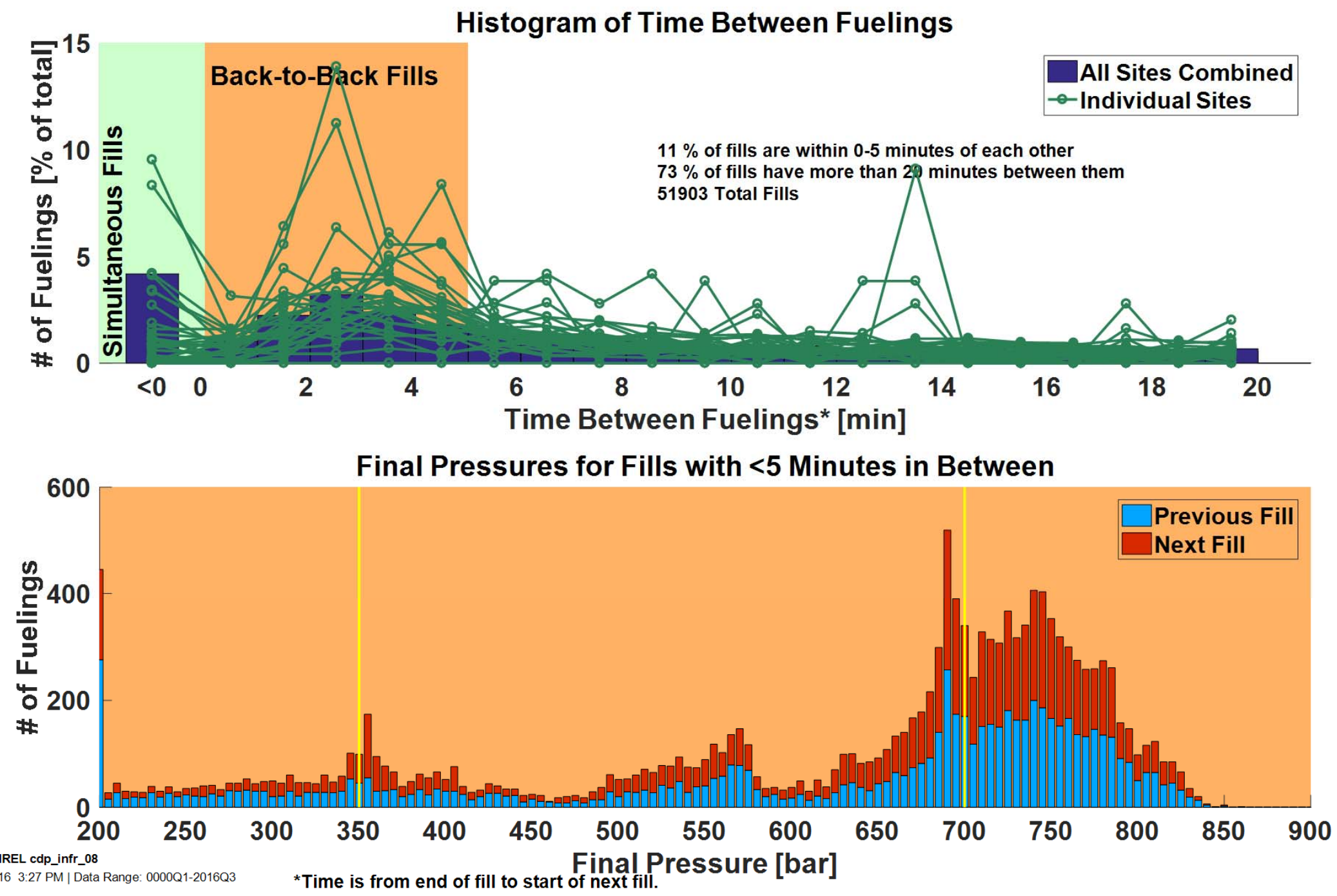


\section{Fueling Final Pressures}
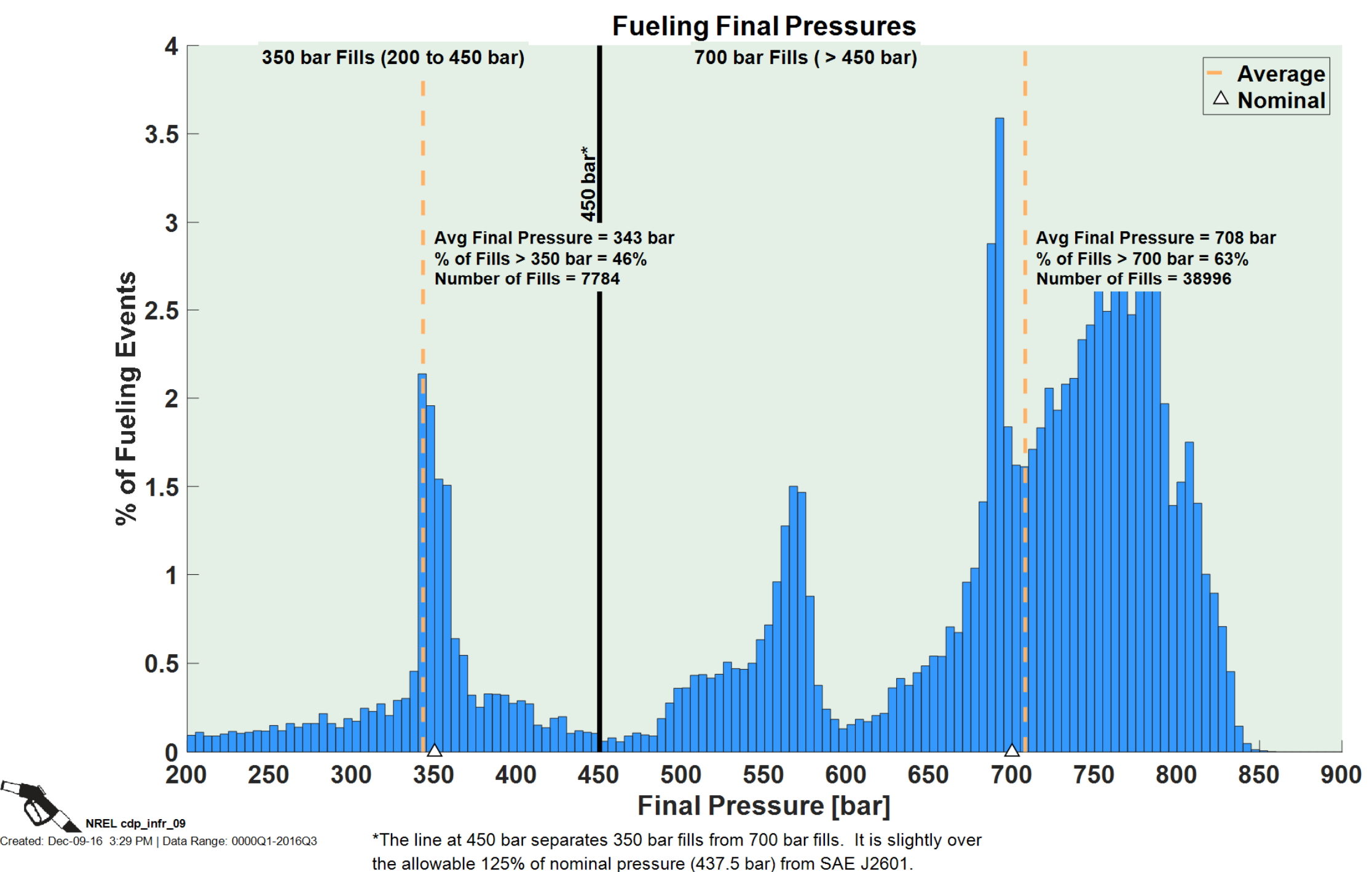
Histogram of Fueling Rates

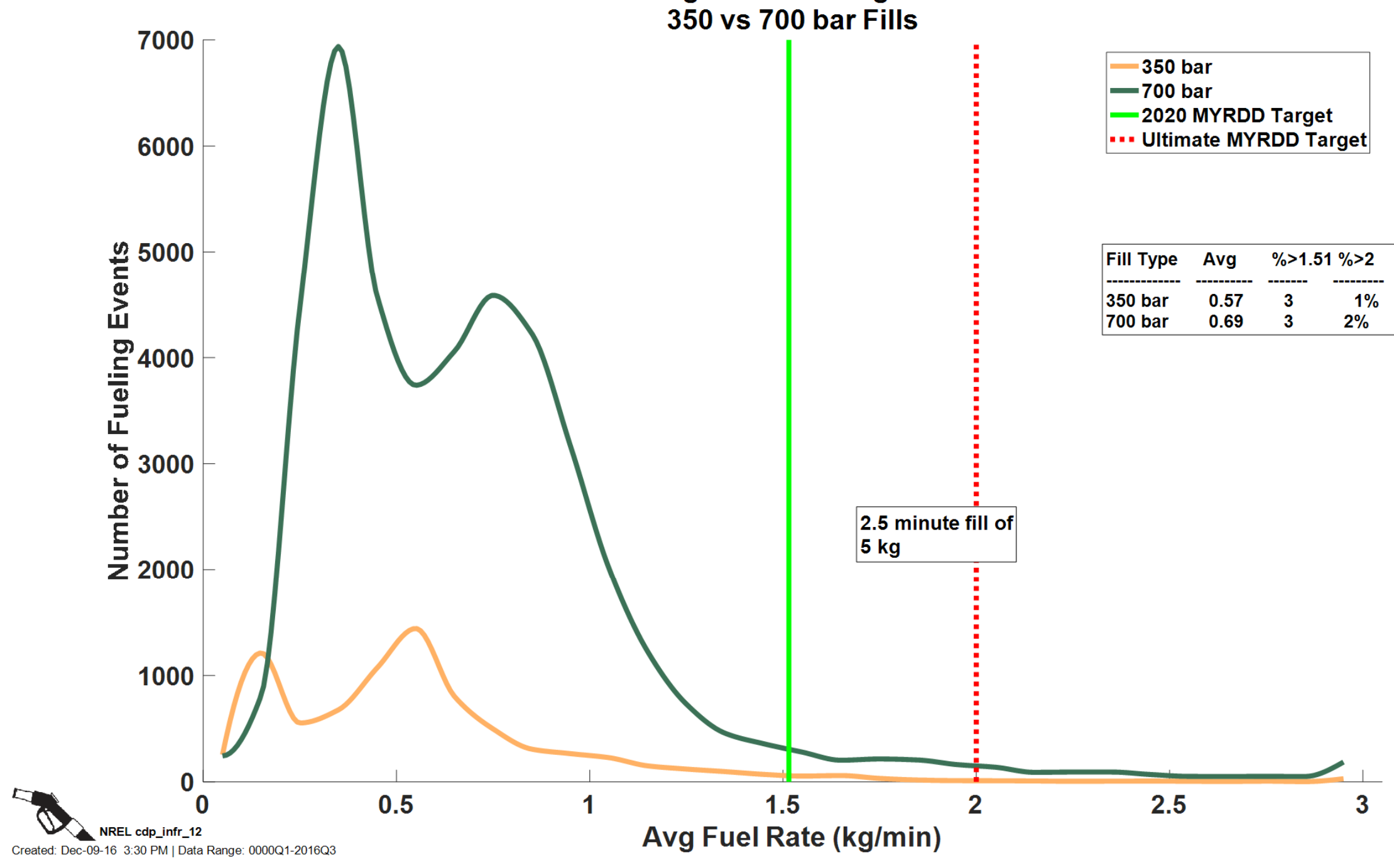




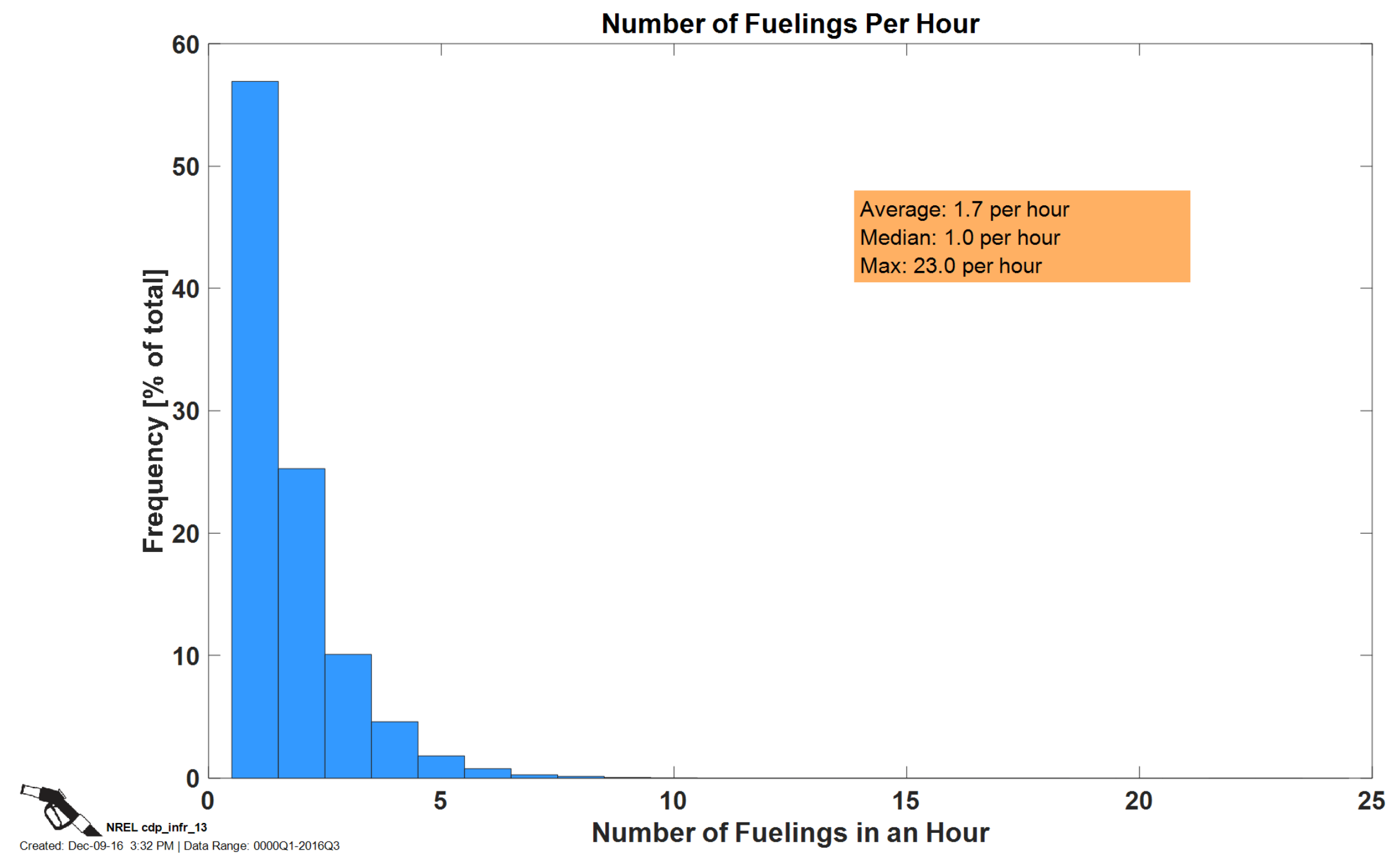




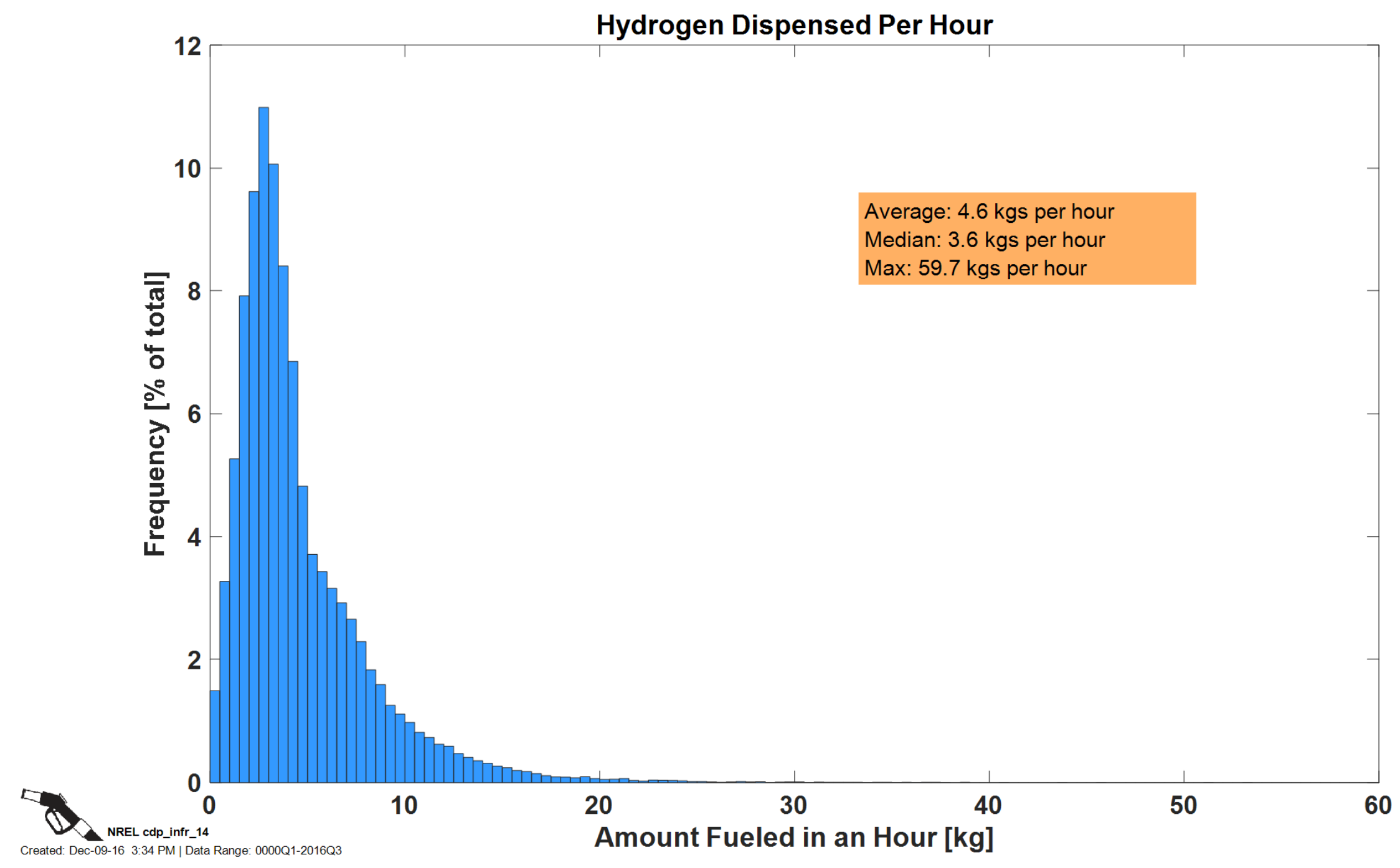




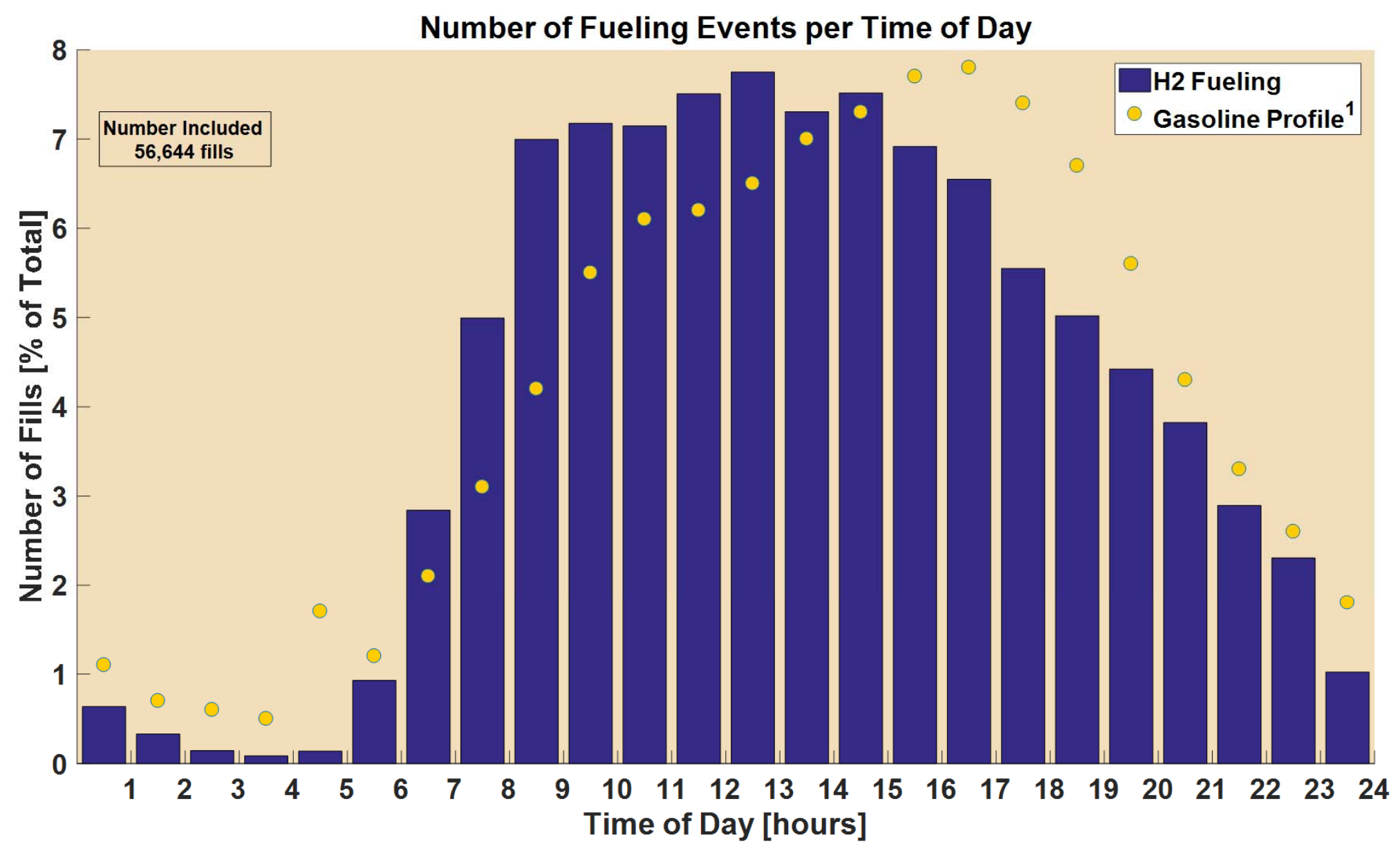




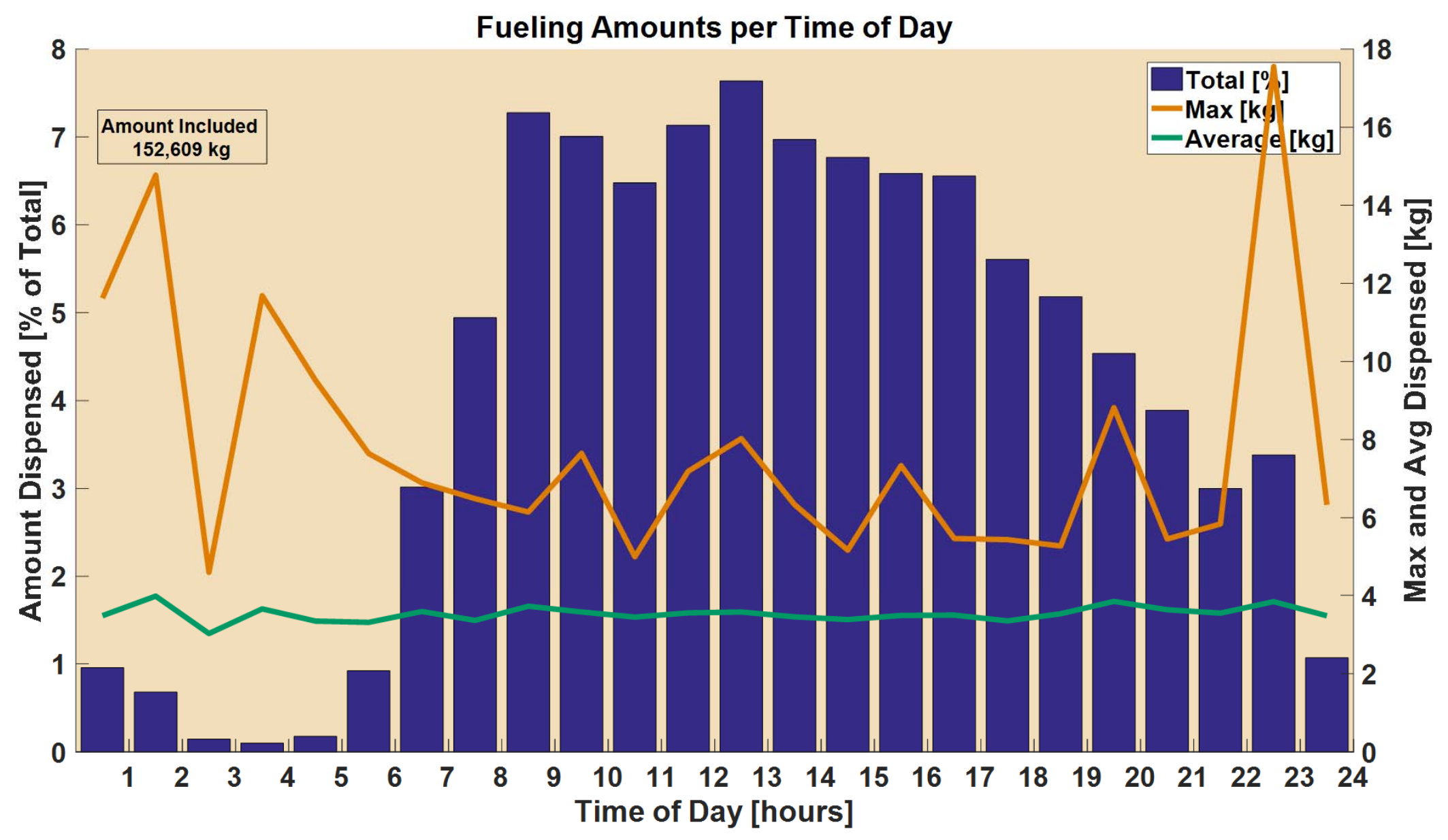




\section{Fueling Rates by Amount Filled}

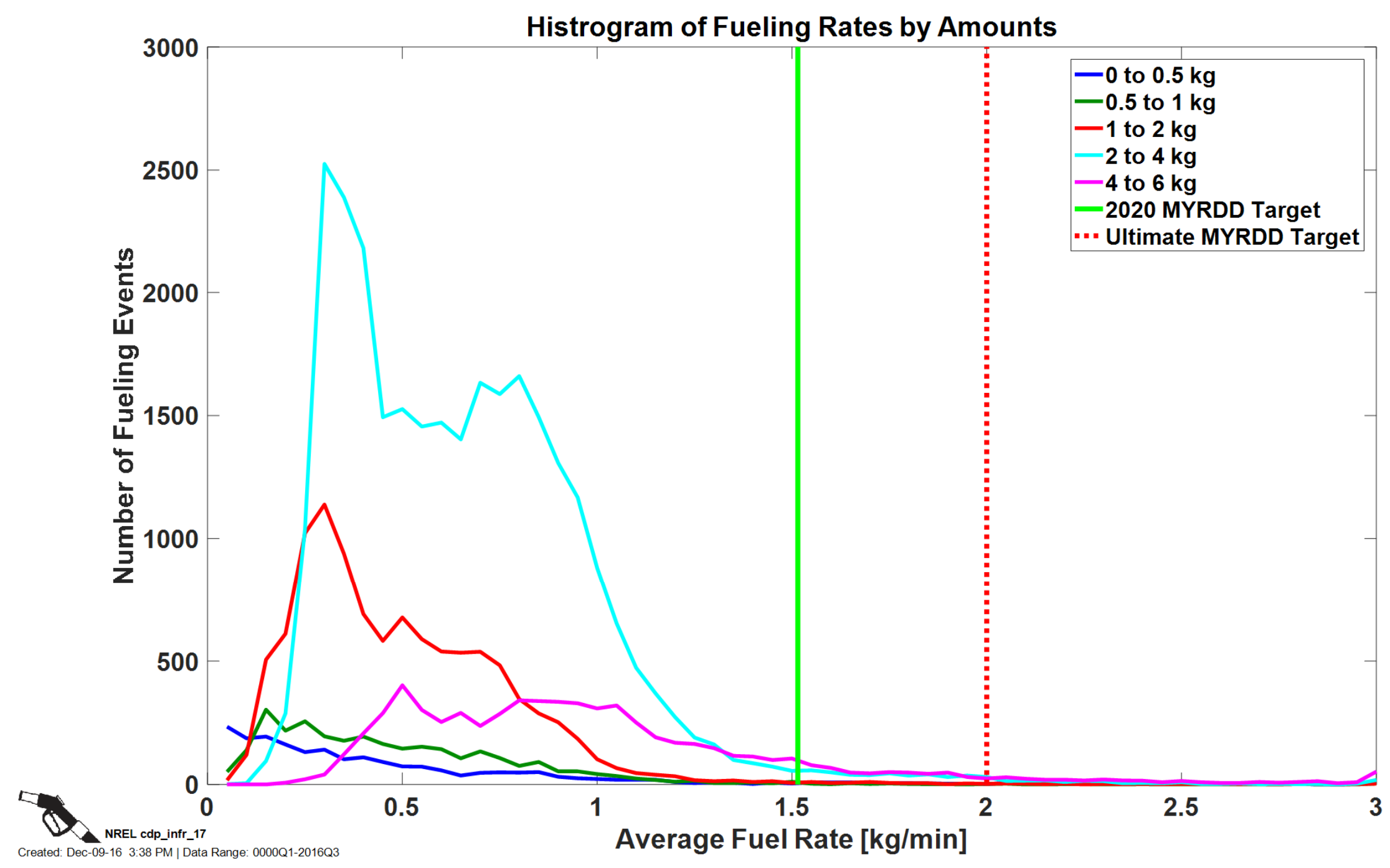




\section{CDP-INFR-18}

Fueling Amount vs. Time to Fill

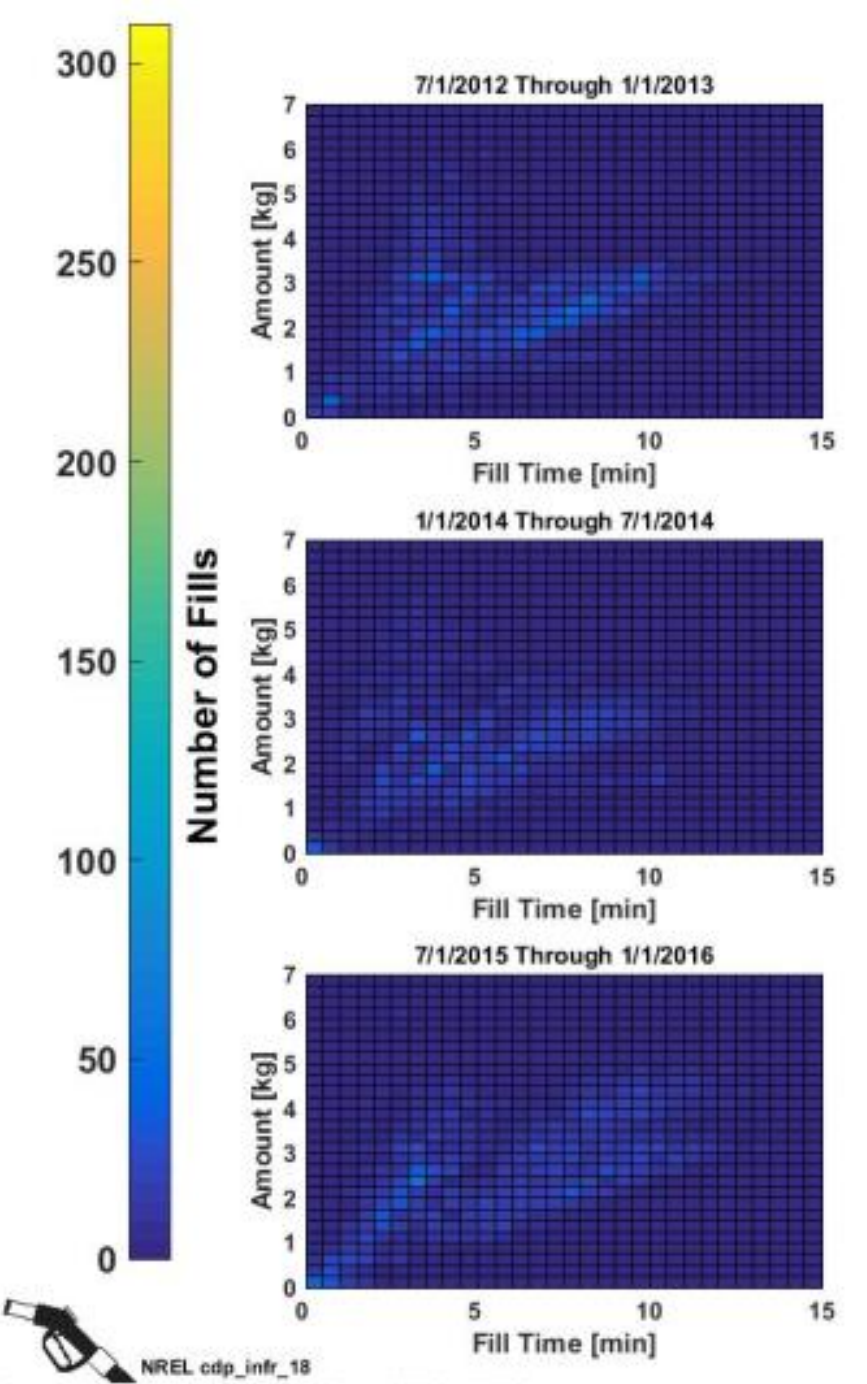

Histrogram of Fueling Amount Vs Time
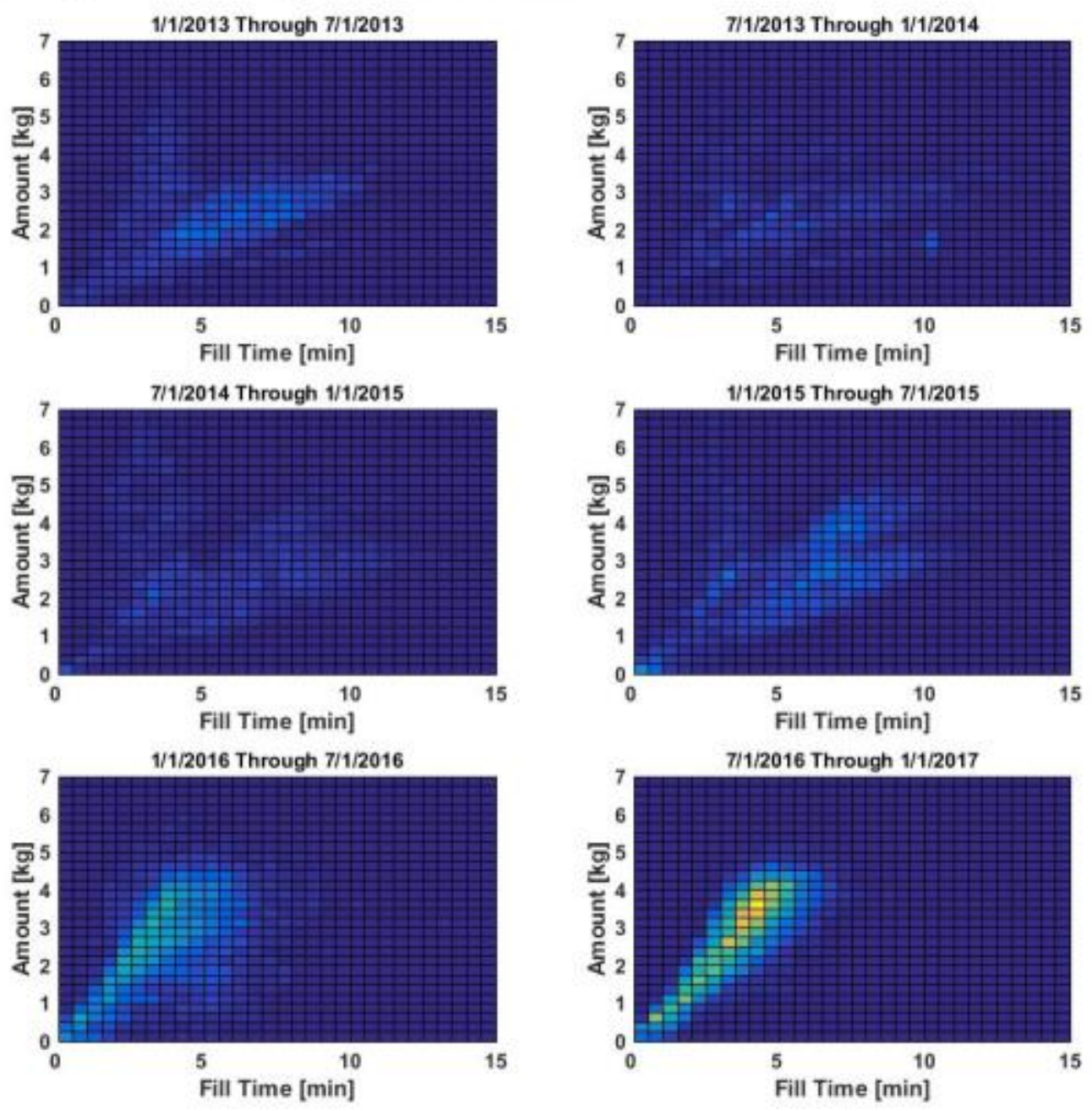


\section{Histogram of Fueling Rates}

By Year

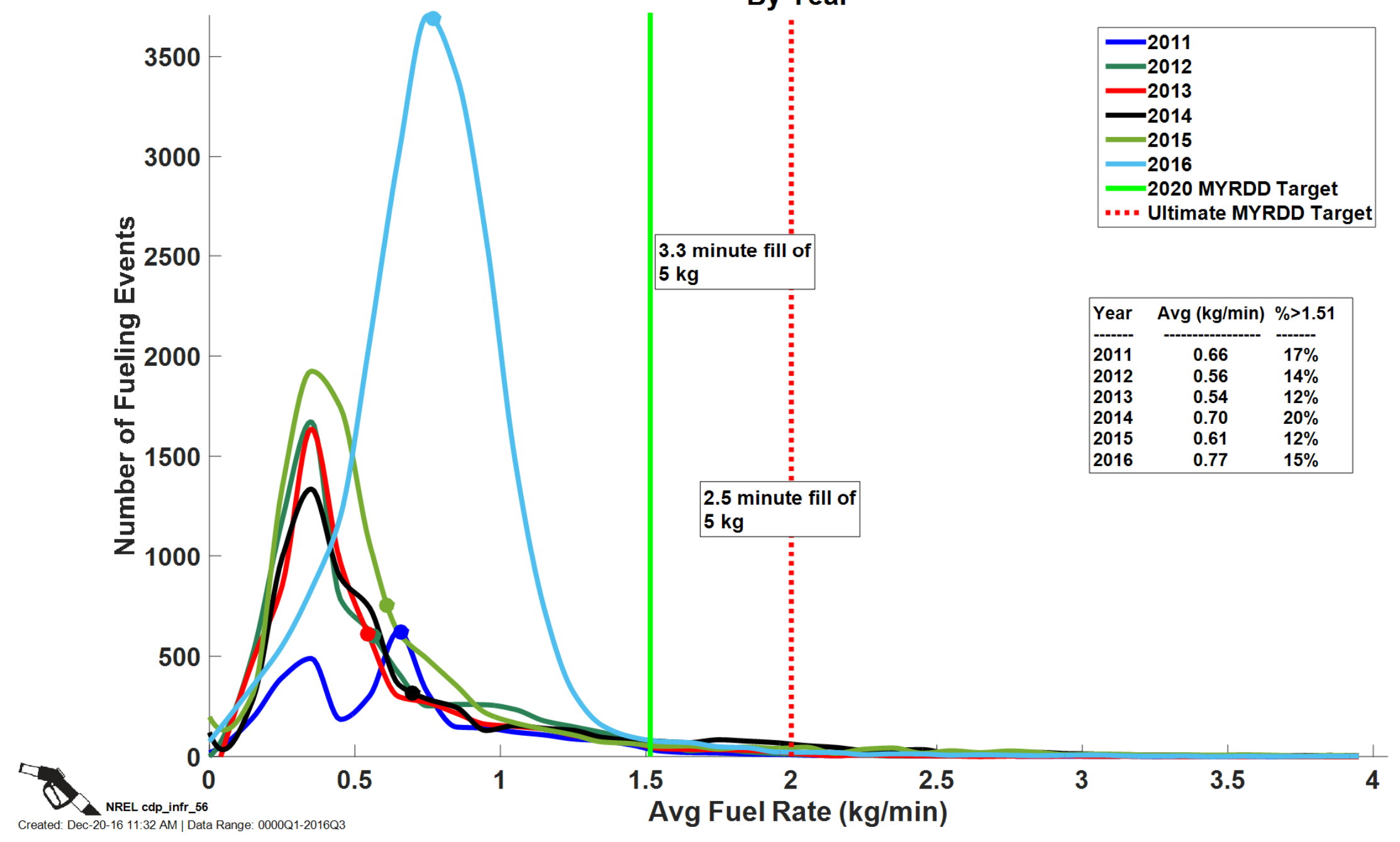




\section{Monthly Averages: 700 bar Fills $>1 \mathrm{~kg}$ with Pre-Cool of $-20^{\circ} \mathrm{C}$}
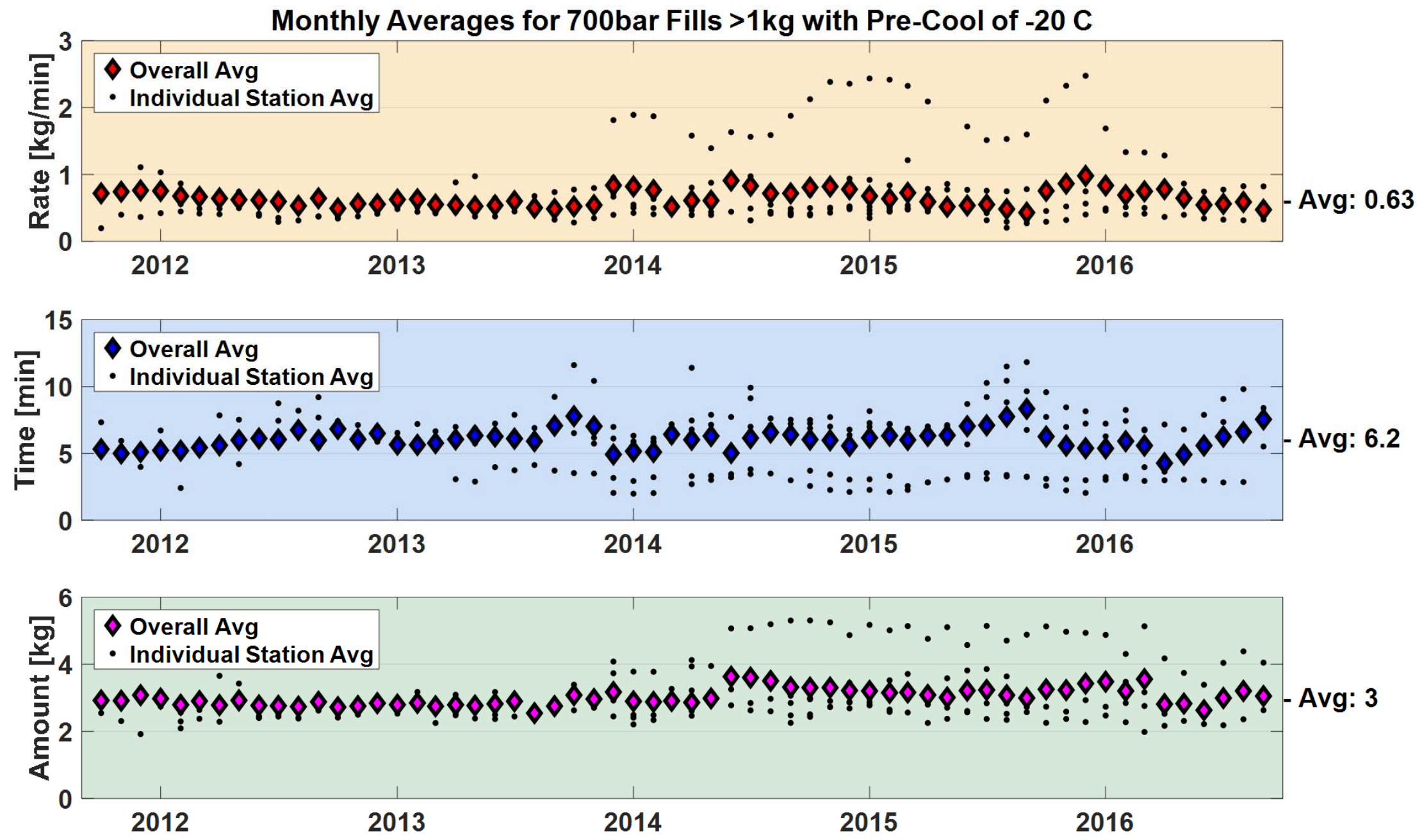


\section{Monthly Averages: All Fills}

Monthly Averages for All Fills
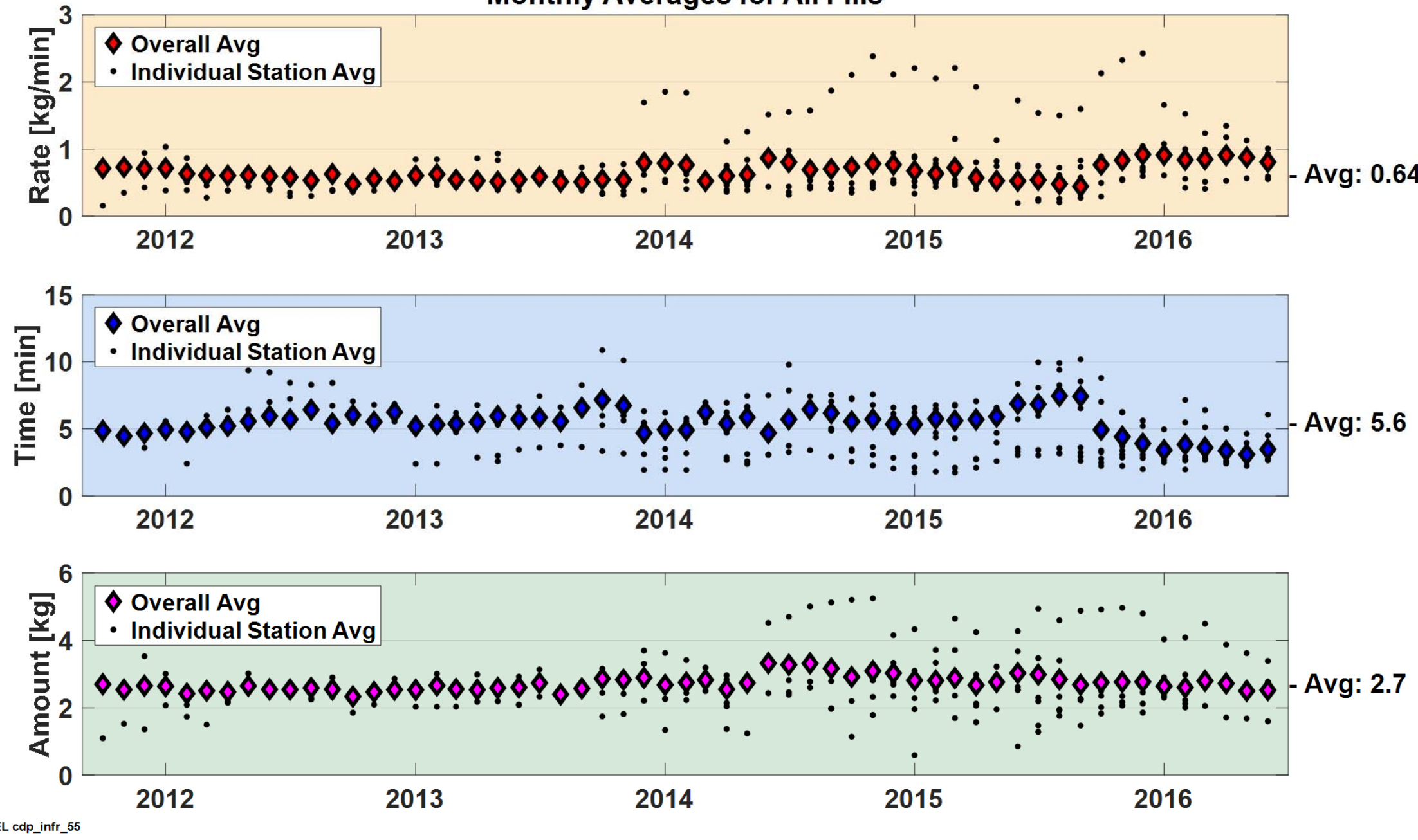

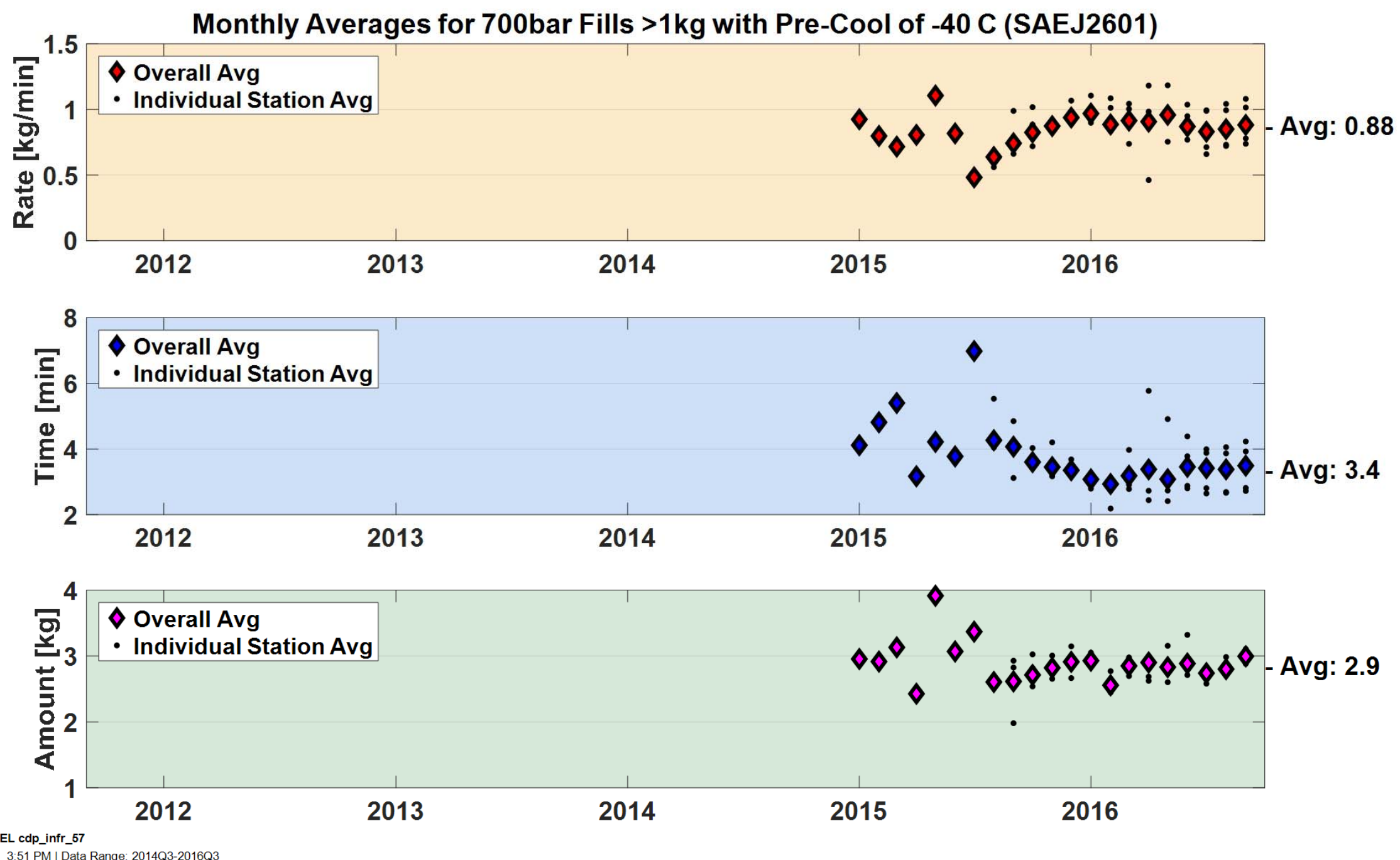


\section{Fuel Temperature at Receptacle $30 \mathrm{~s}$ After Start of Fill}

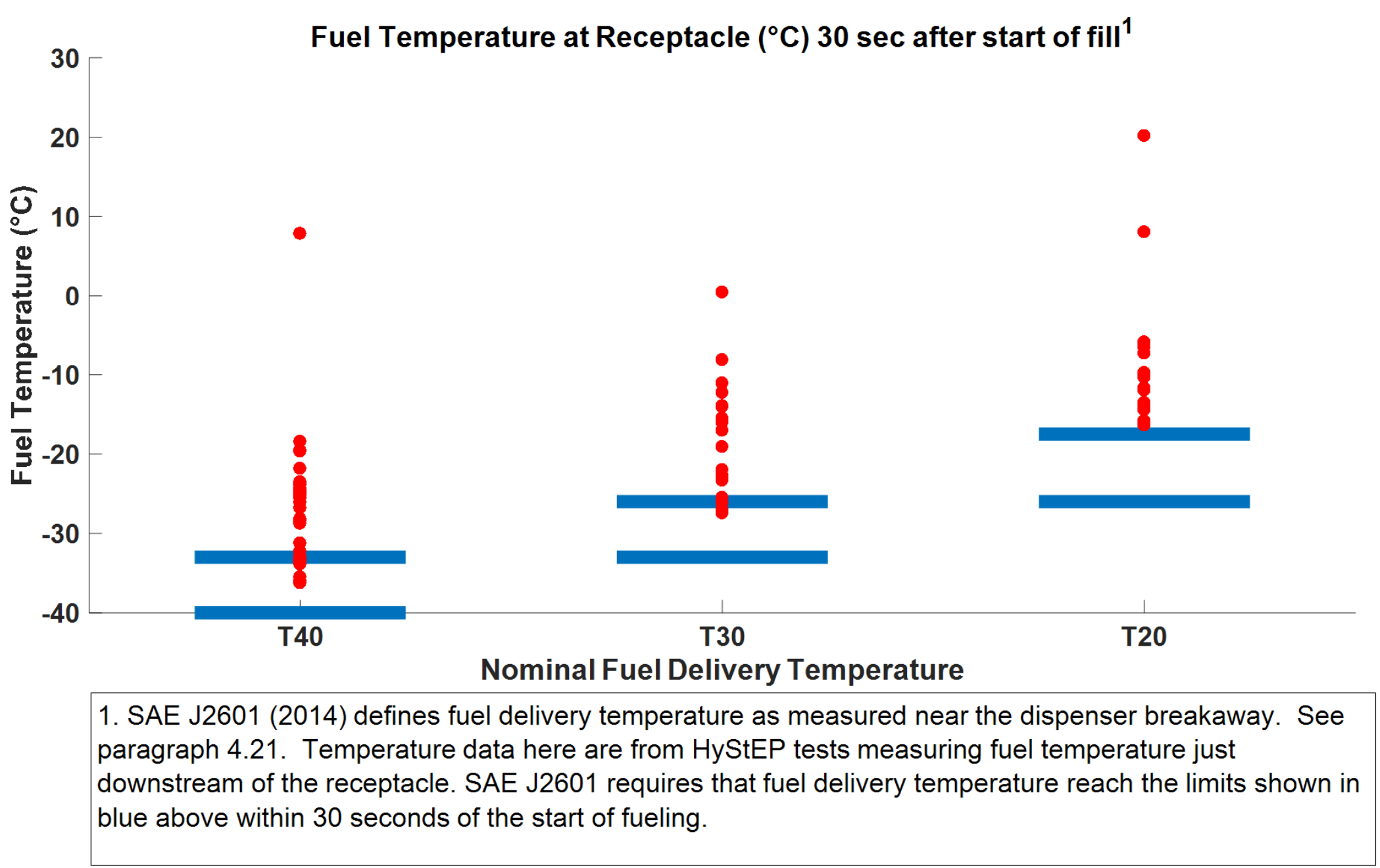


Cost 


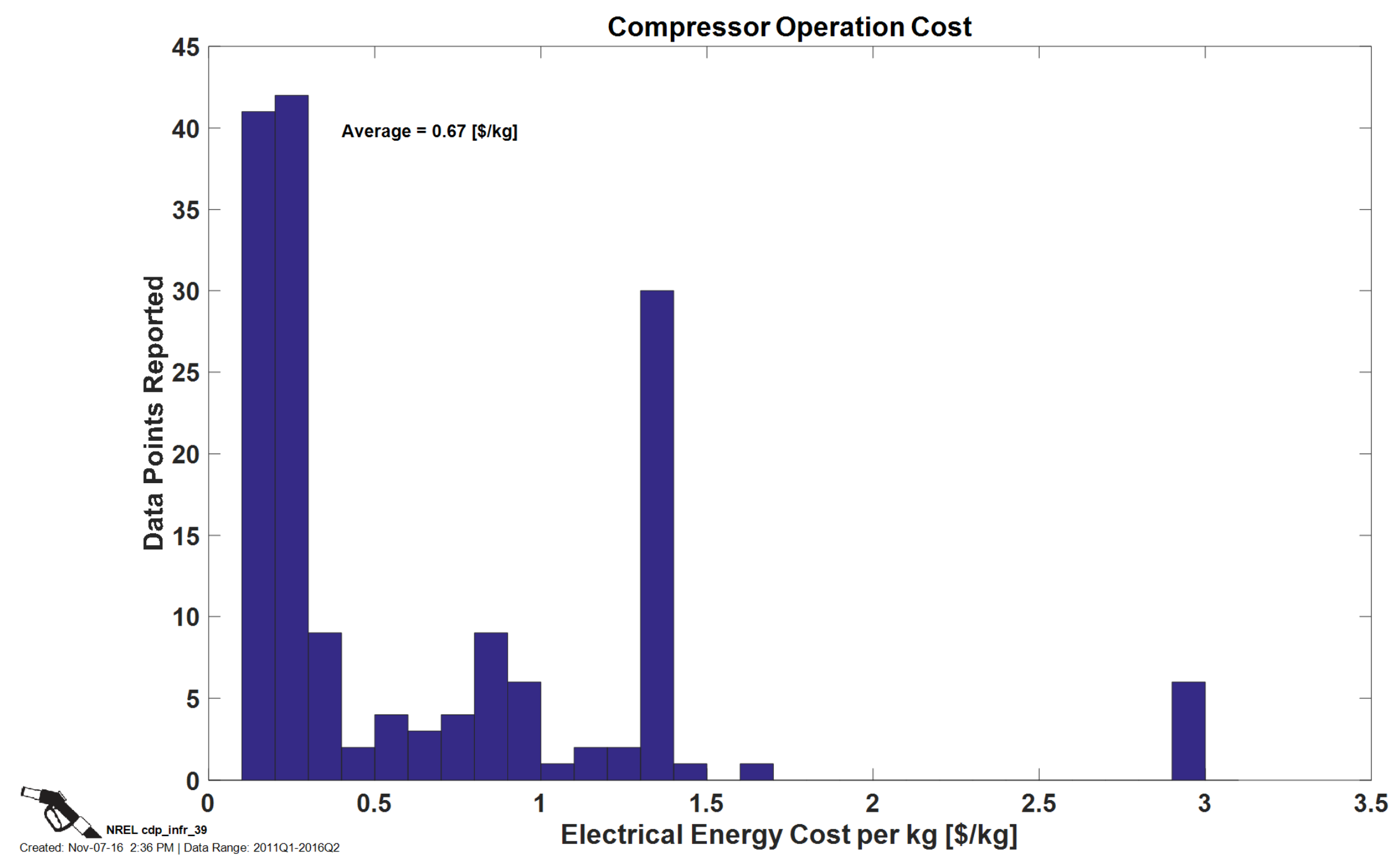




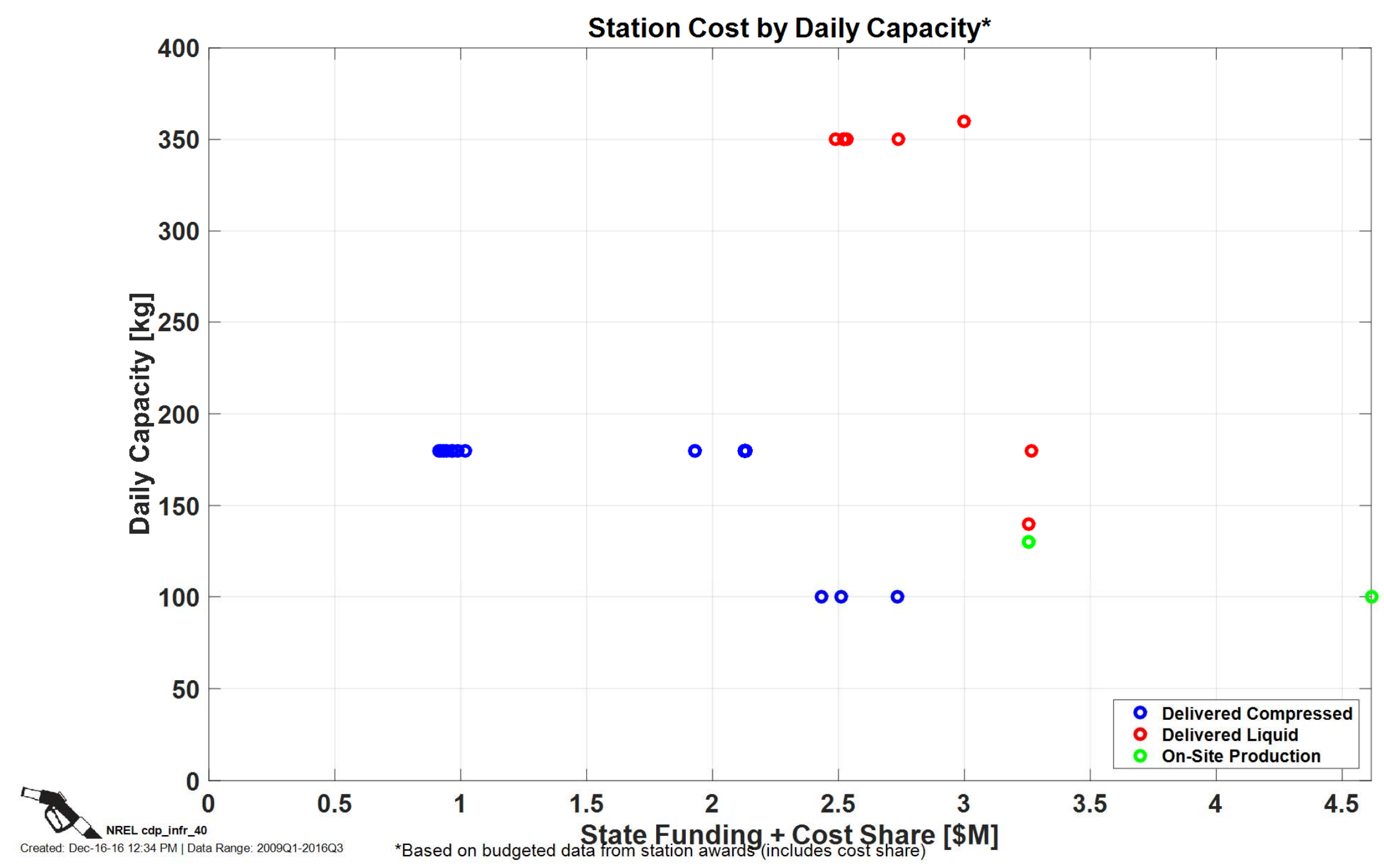




\section{Average Station Cost by Category}

\section{Budget Amounts* $($ Avg Total $=\$ 2.2 \mathrm{M}), 46$ Stations}

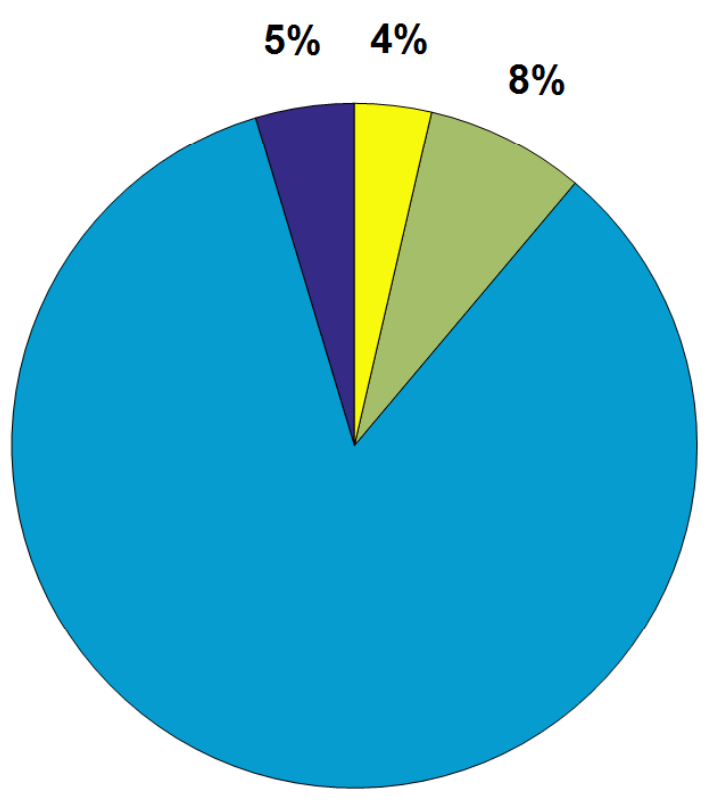

General \& Administration

Station**

Commissioning

Data Reporting ${ }^{* * *}$

\section{$84 \%$}

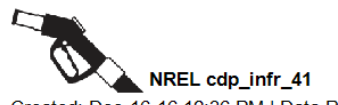

Created: Dec-16-16 12:36 PM | Data Range: 2009Q1-2016Q3
*Based on budgeted data from station awards (includes cost share)

${ }^{*}$ Station includes: Hydrogen Equipment and Station Engineering, Design, Fabrication, Procurement, Site Preparation, Installation, and Construction

***Data Reporting includes quarterly reporting on performance, operation and maintenance 


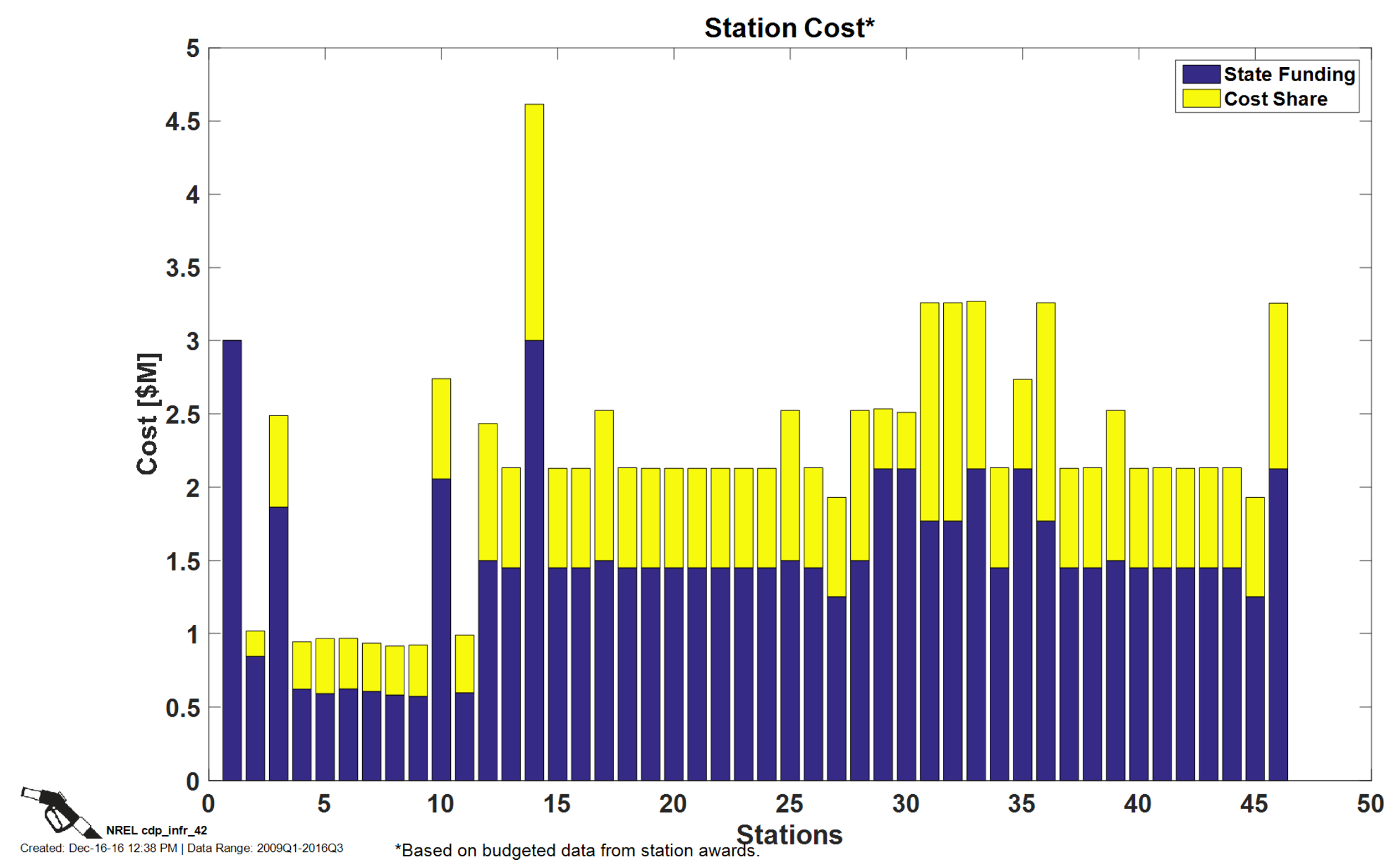




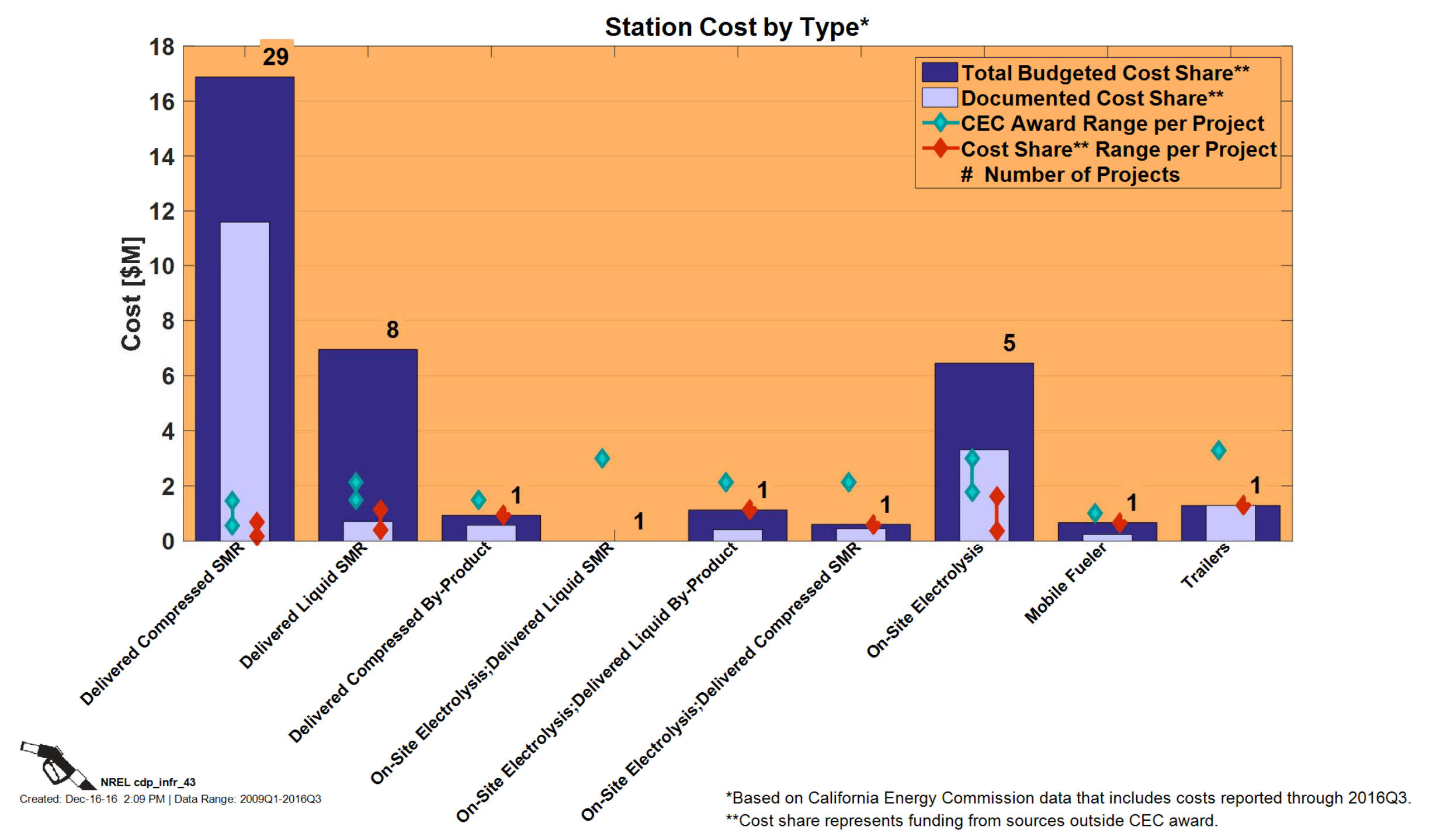




\section{Monthly Maintenance Costs}

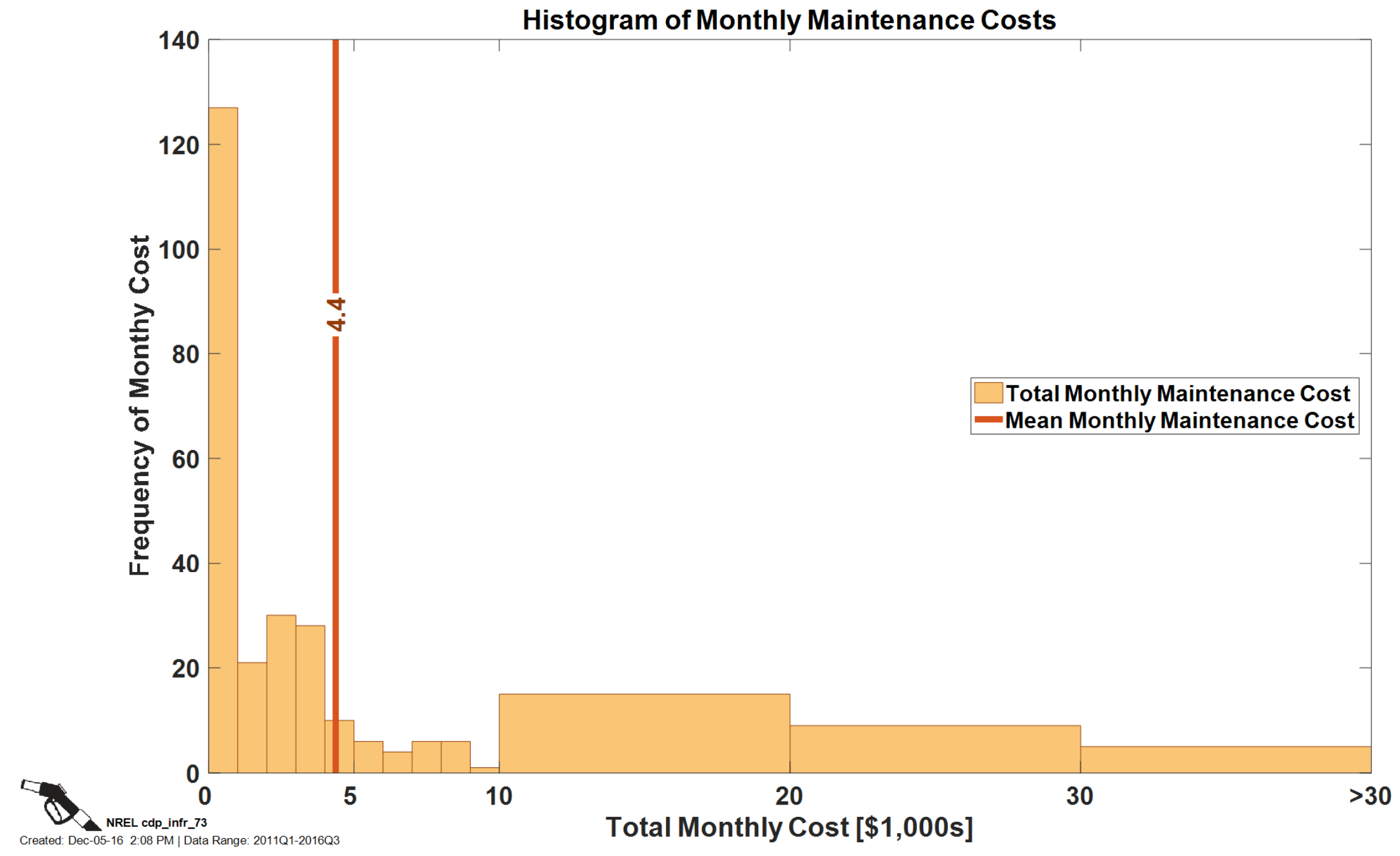




\section{Maintenance Cost per kg of Hydrogen Dispensed}

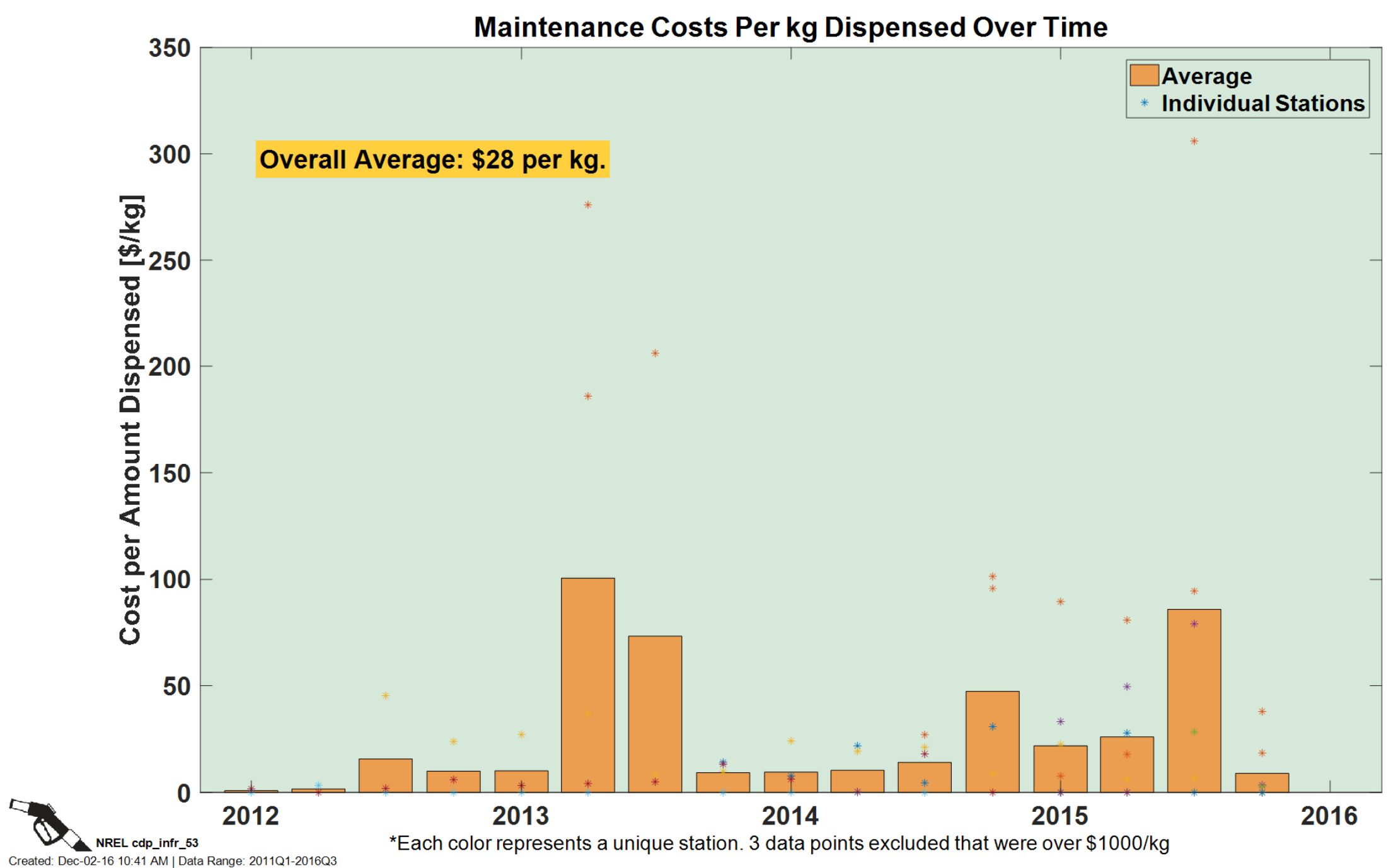




\section{Utilization}




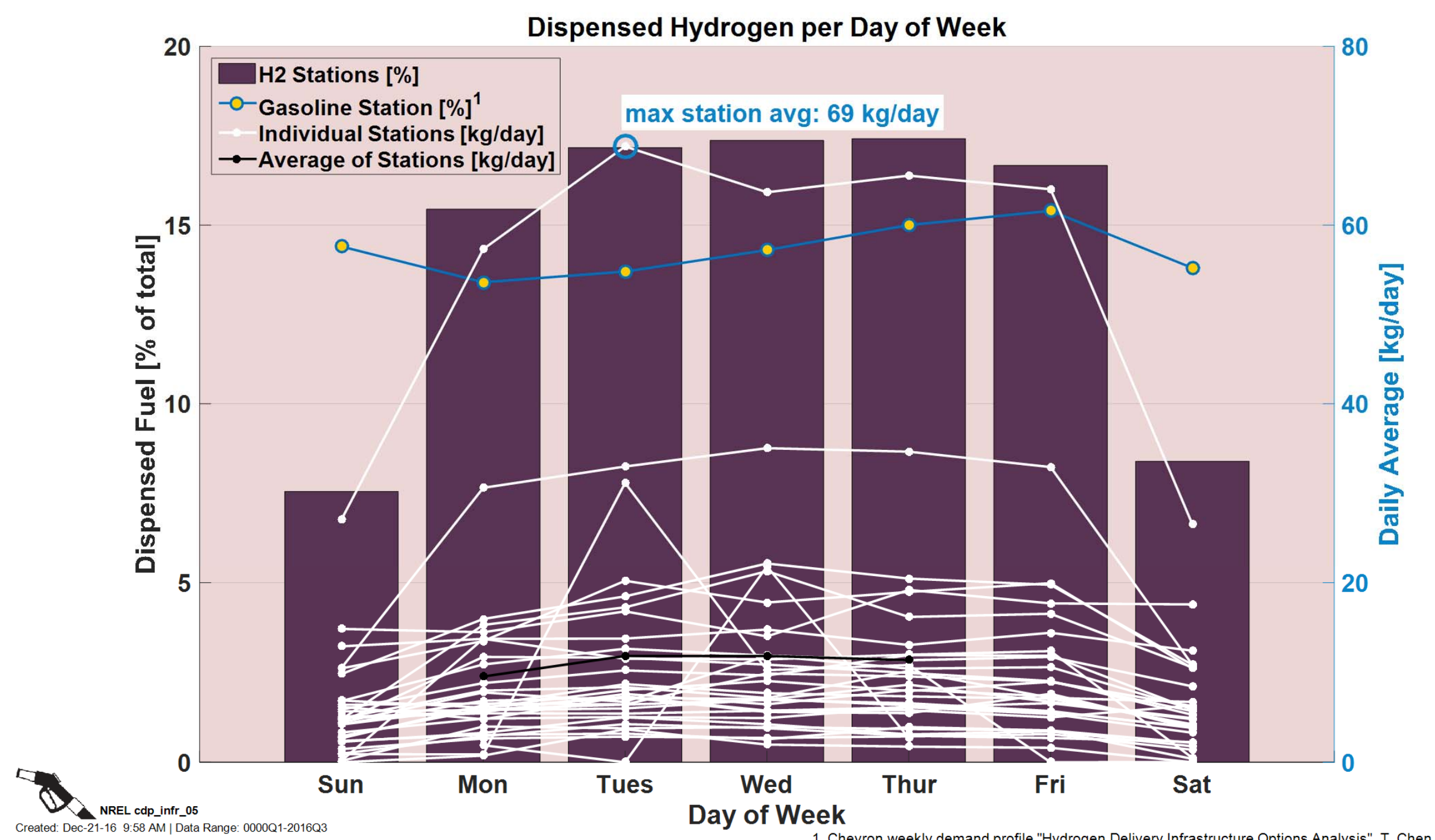




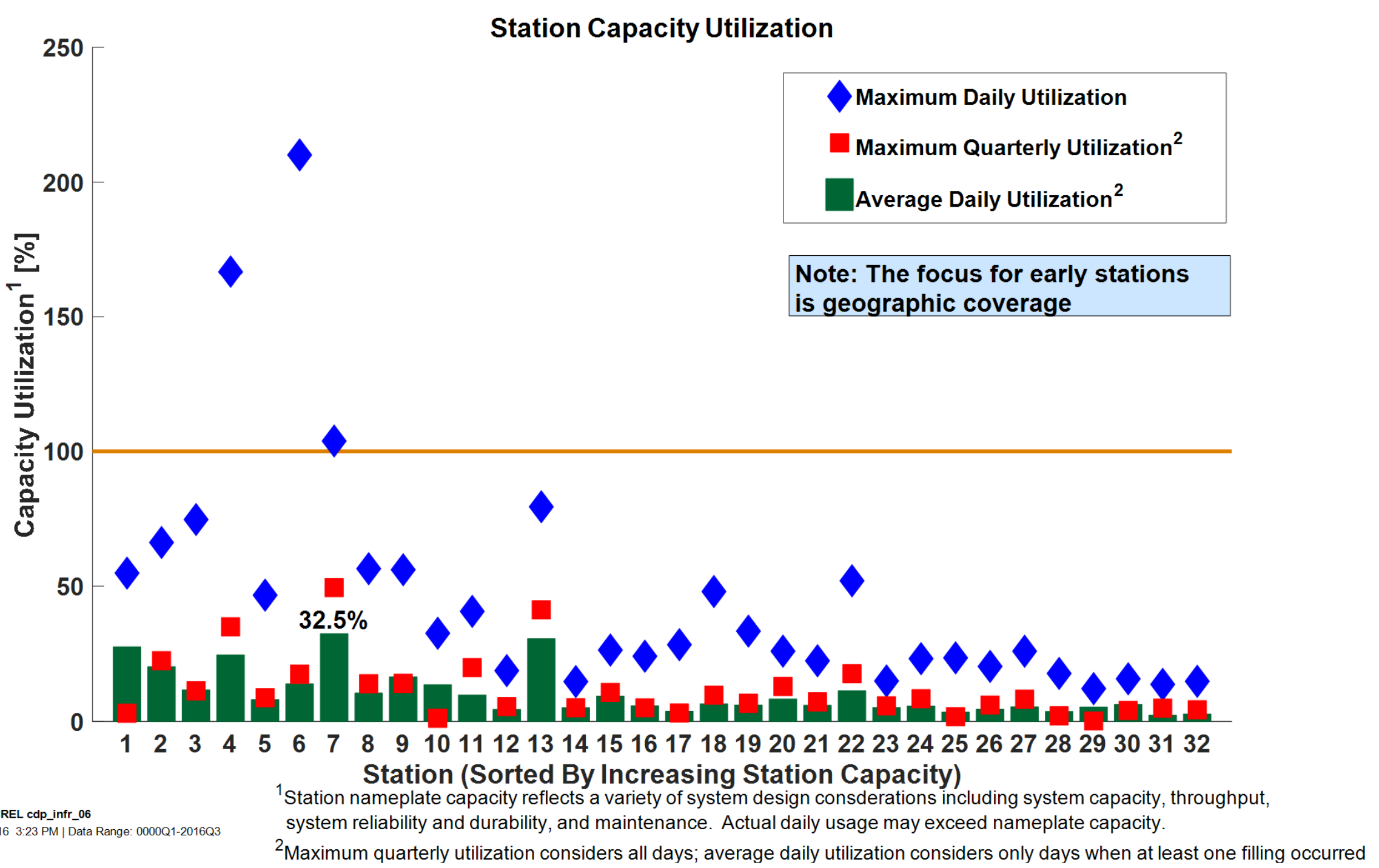




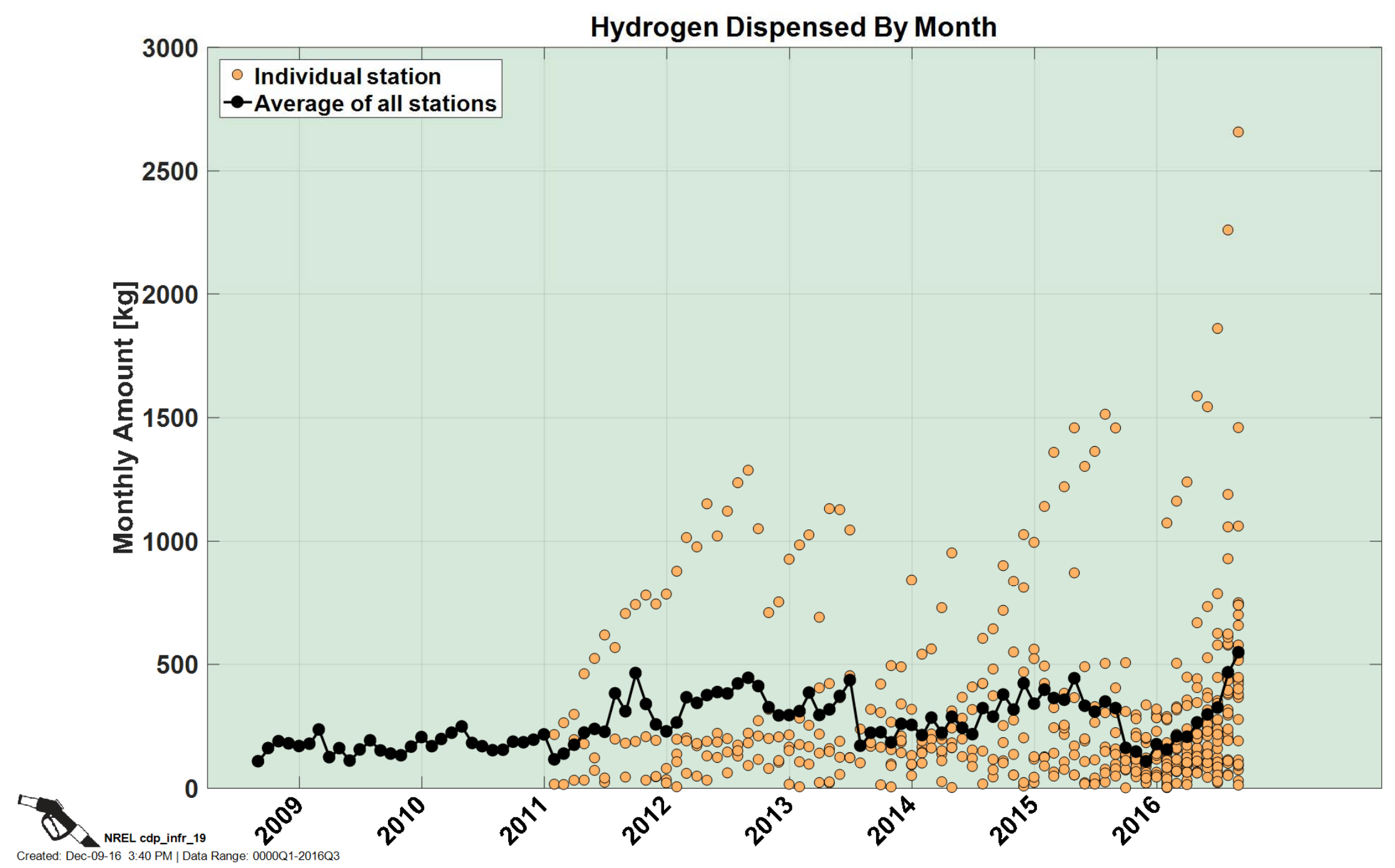




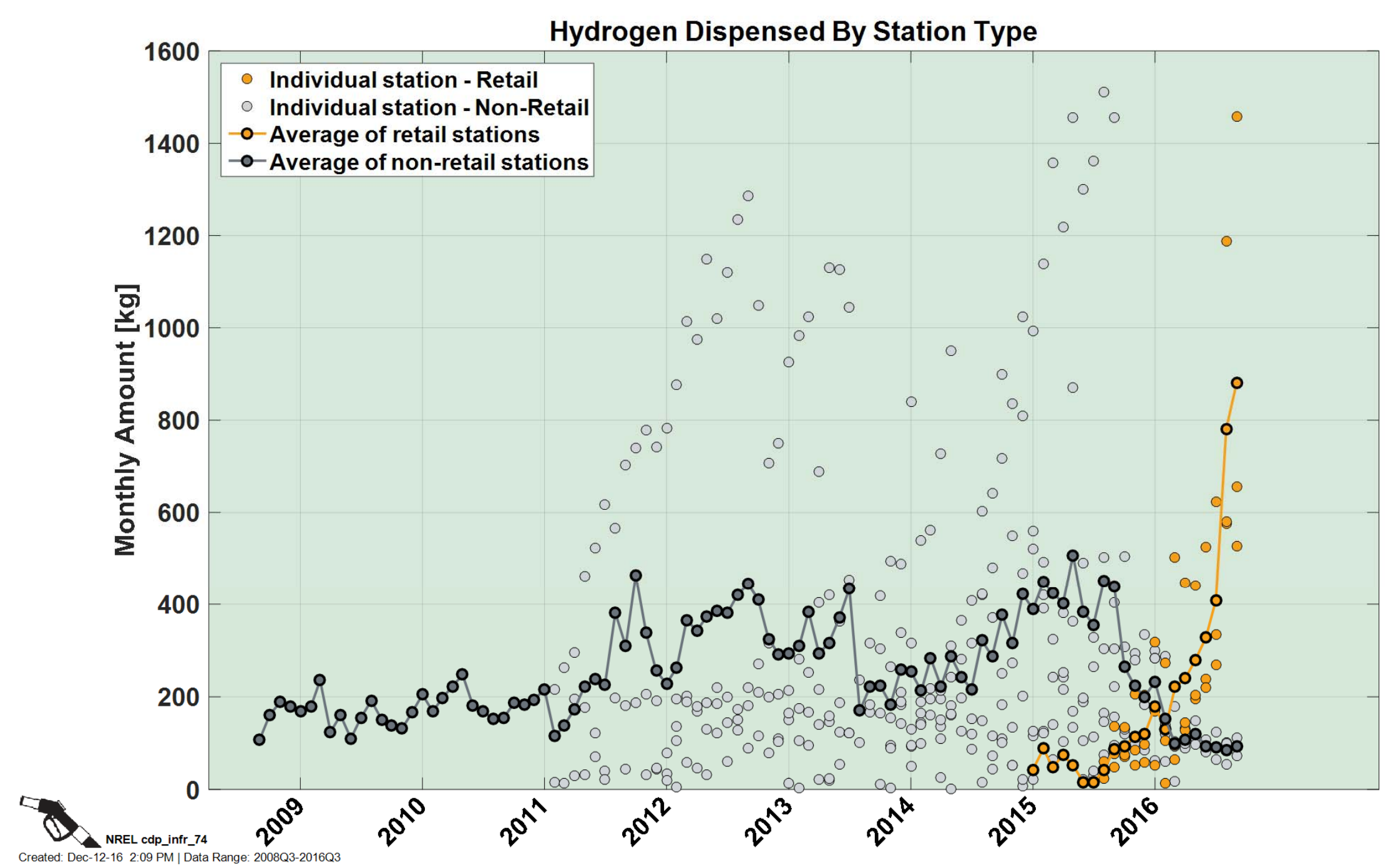




\section{Station Capacity Utilization Trends by Quarter}

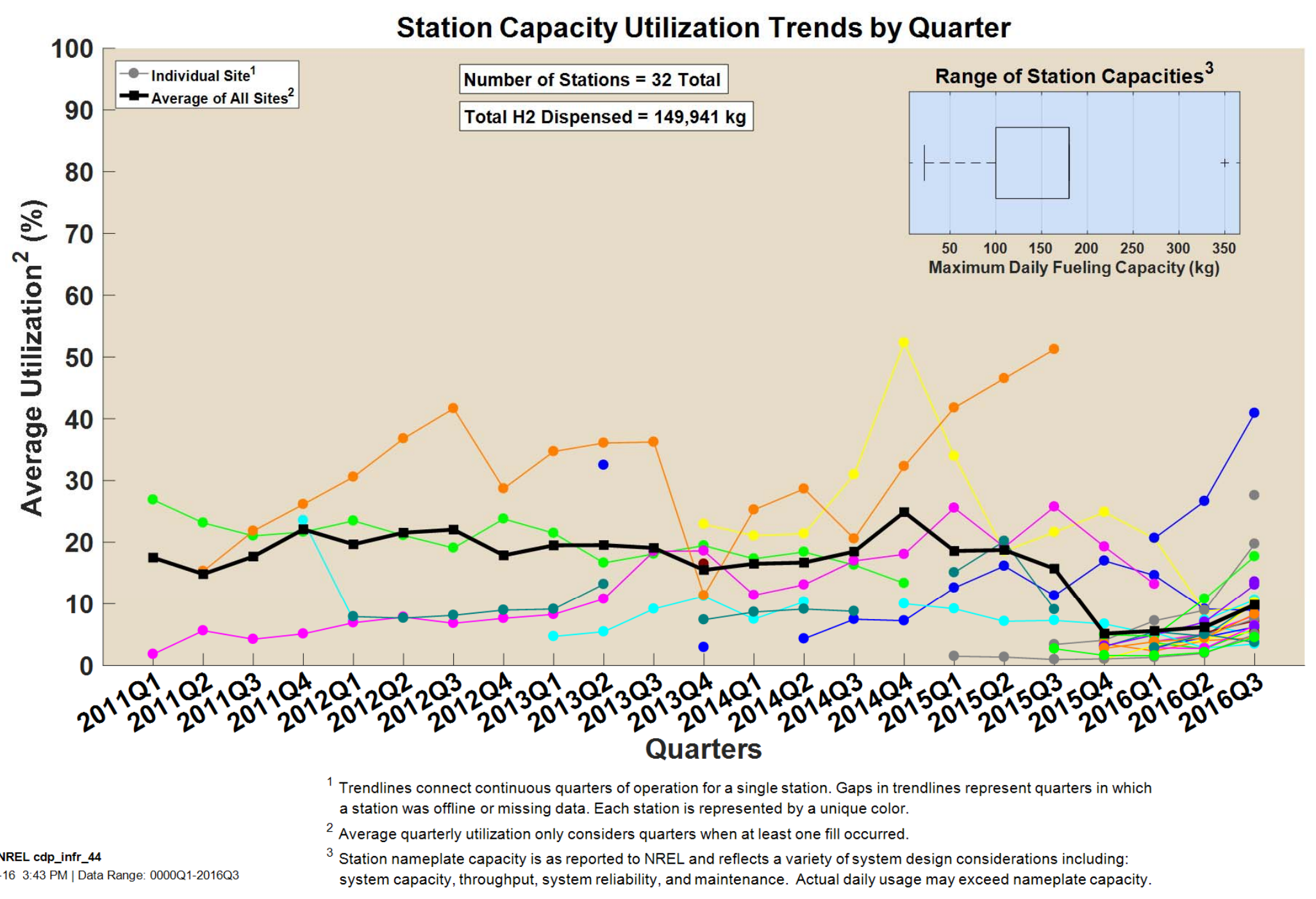




\section{Station Amount Dispensed by Quarter}

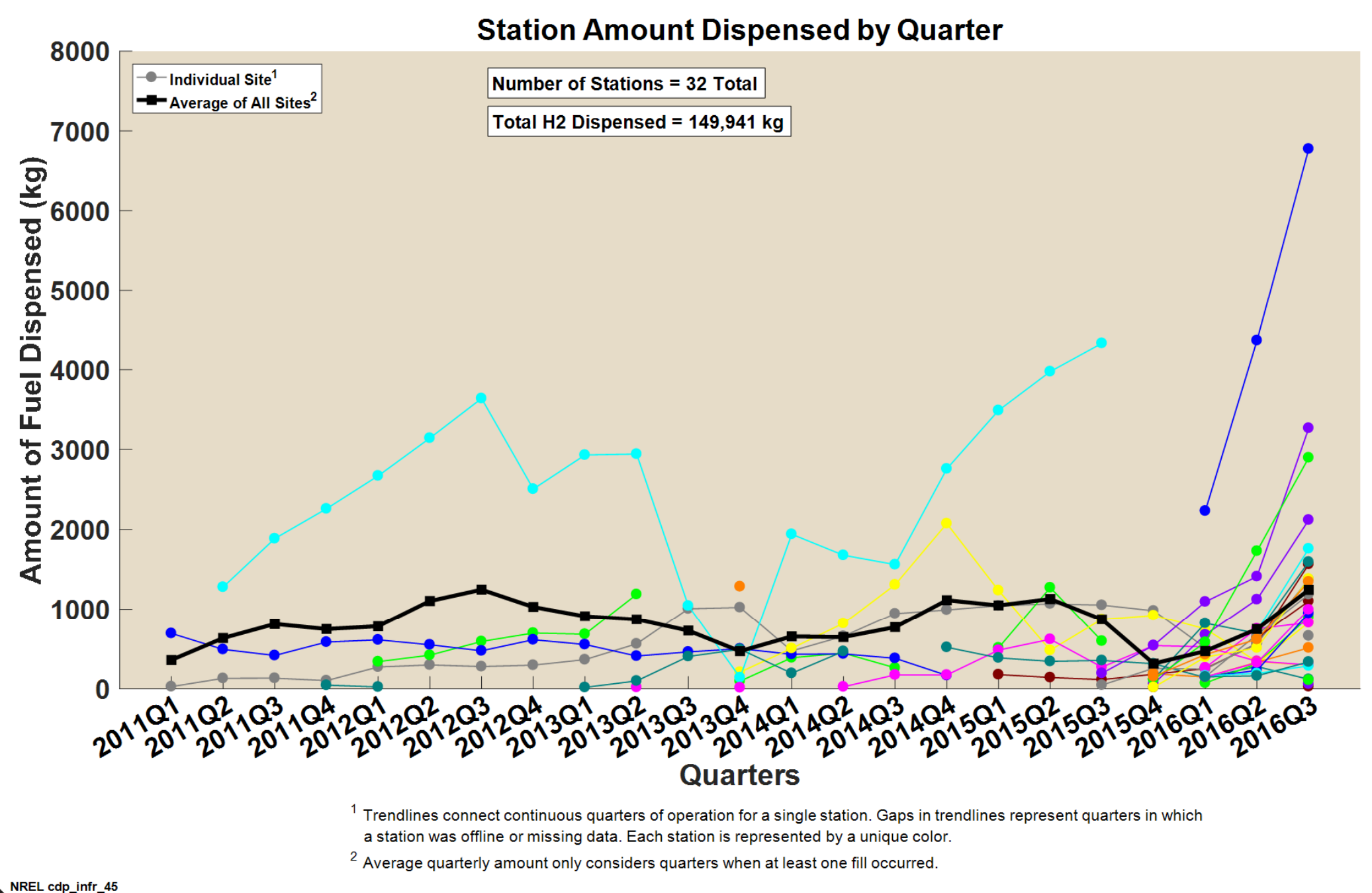




\section{Days with Fills by Quarter}

- Individual Site - -Average of All Sites ${ }^{2}$ Number of Stations $=32$ Total

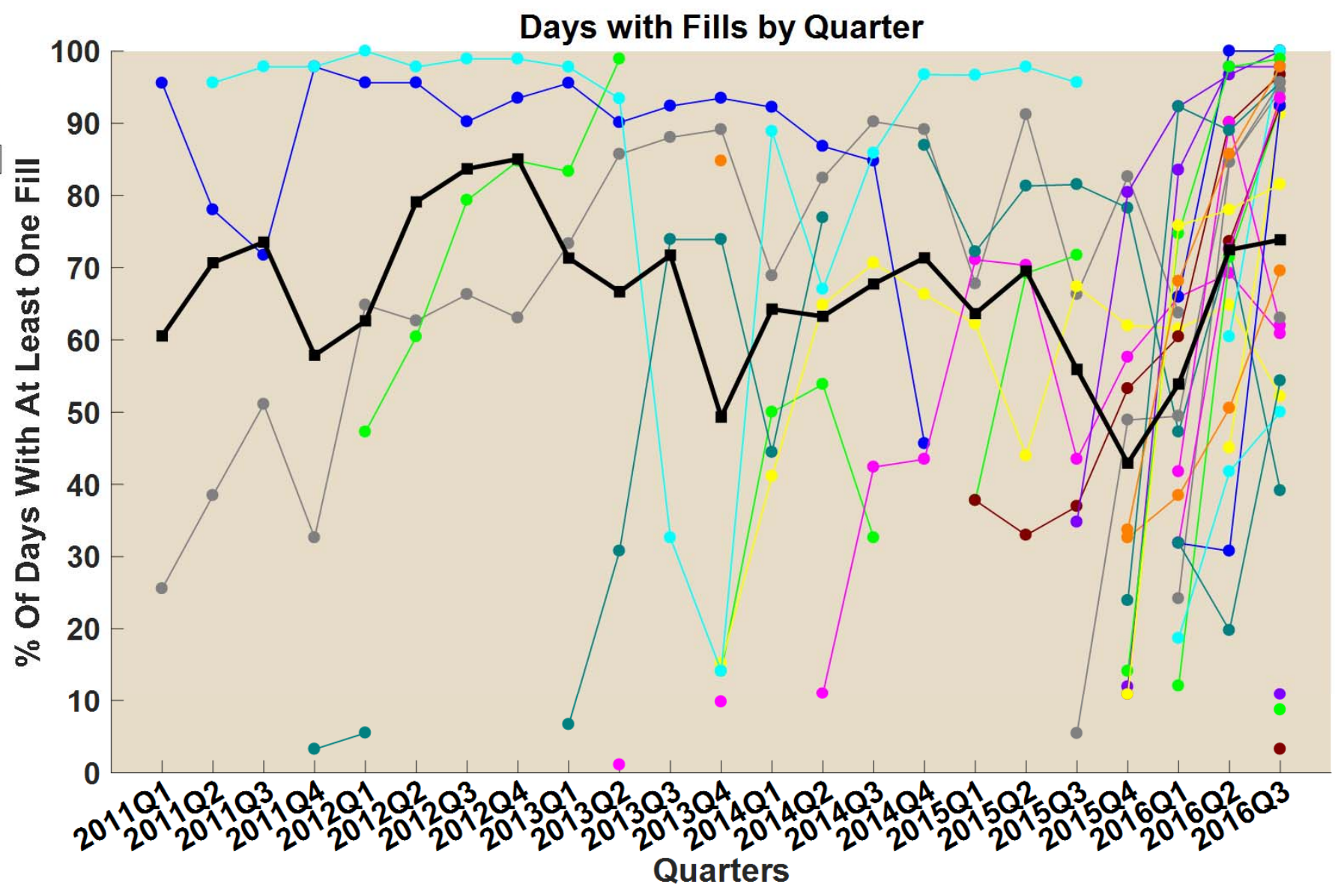

${ }^{1}$ Trendlines connect continuous quarters of operation for a single station. Gaps in trendlines represent quarters in which a station had no fills or was missing data. Each station is represented by a unique color.

2 The average percent of days with fills only considers quarters in which at least one fill occurred. Stations with no filling days in a quarter are excluded from the average for that quarter. All stations with at least one fill in a quarter are given equal weight when calculating the average for the quarter. 


\section{Summary of Station Usage Statistics ${ }^{4}$}
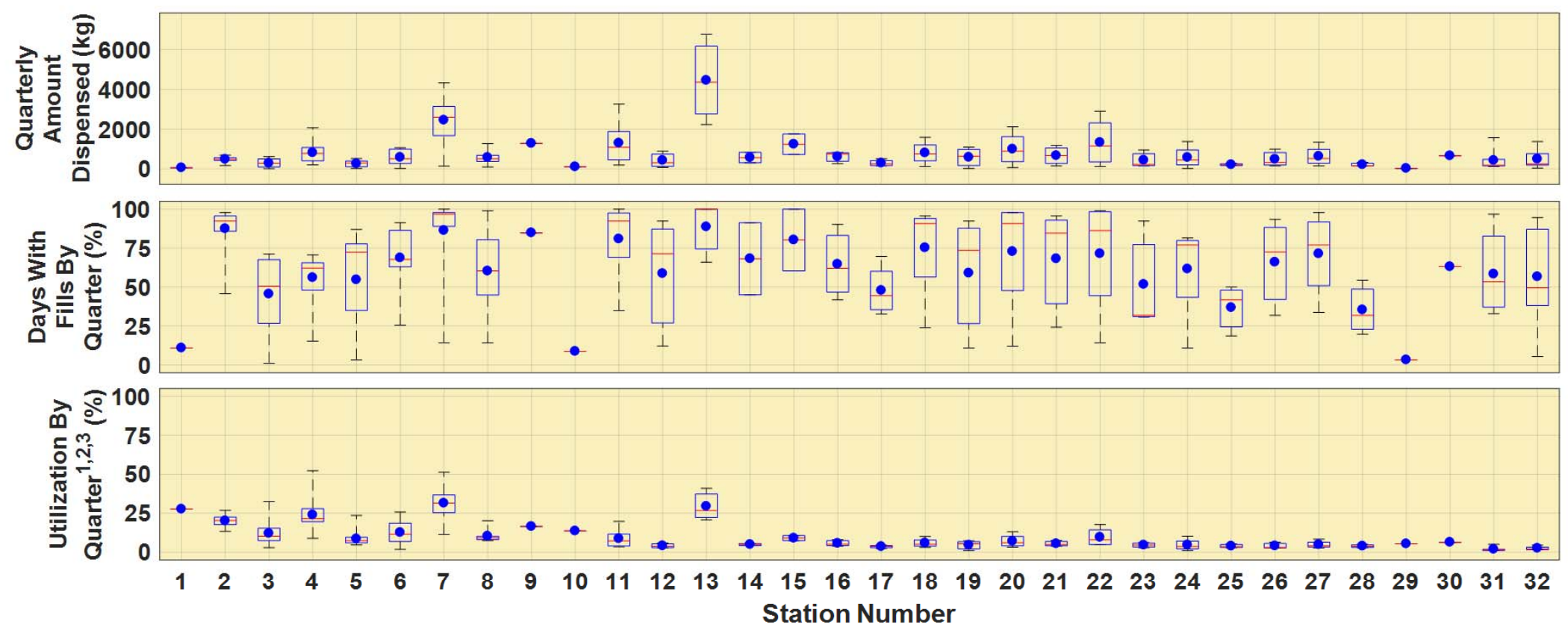

${ }^{1}$ Station nameplate capacity is as reported to NREL and reflects a variety of system design considerations including: system capacity, throughput, system reliability, and maintenance. Actual daily usage may exceed nameplate capacity.

${ }^{2}$ Average quarterly utilization only considers days when at least one fill occured.

${ }^{3}$ Utilization is calculated by dividing the quarterly amount dispensed by the stations nameplate capacity.

${ }^{4}$ Only quarters with fills are included.

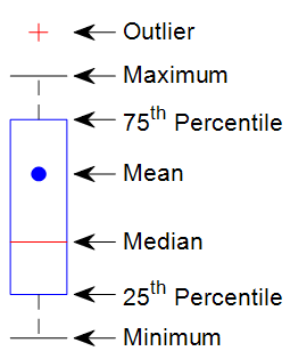

NREL_cdp_infr_47

Created: Dec-09-16 3:47 PM | Data Range: 0000Q1-2016Q3 
Hydrogen Quality 


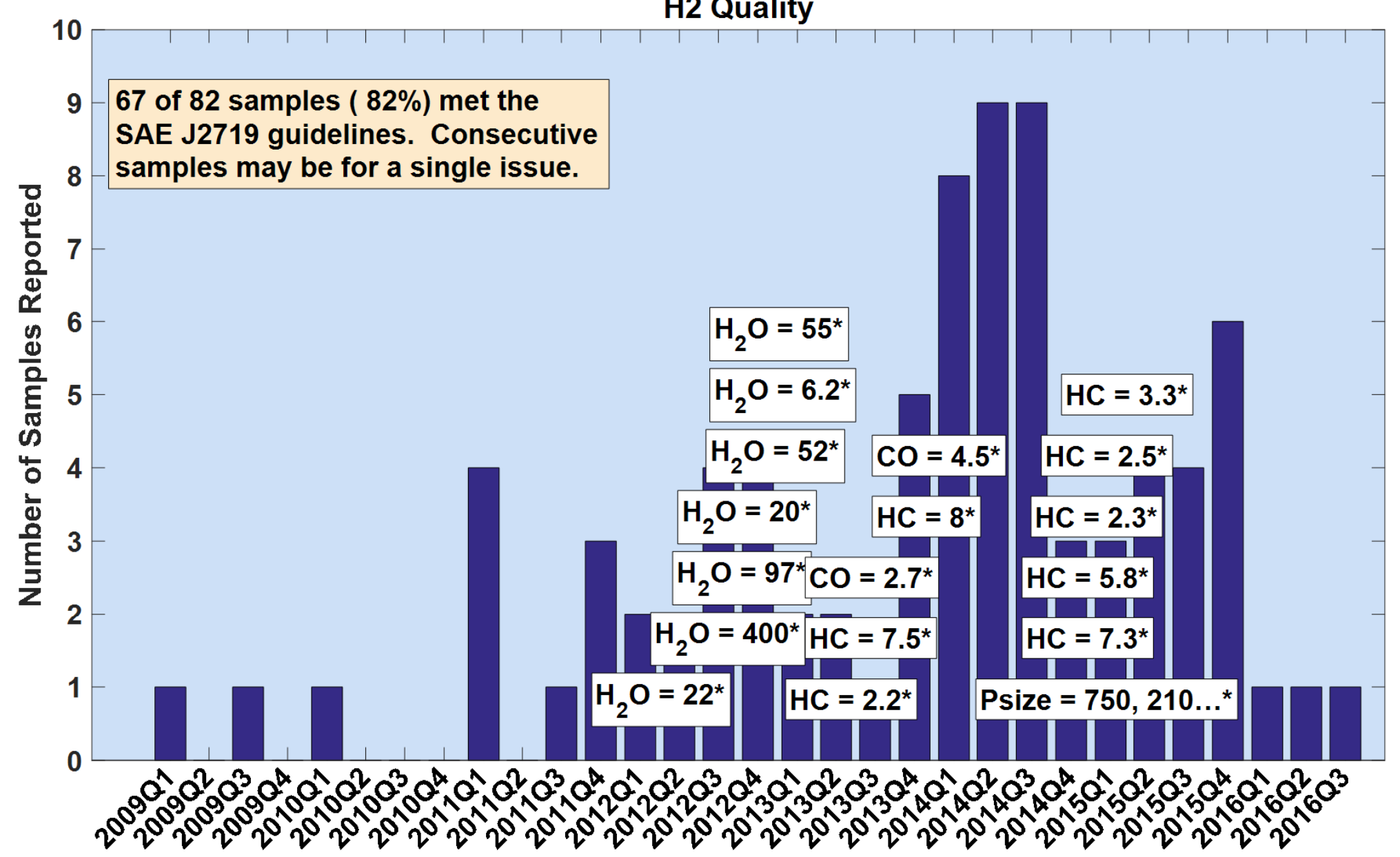




\section{Impurities-Ammonia}

Impurities - Ammonia

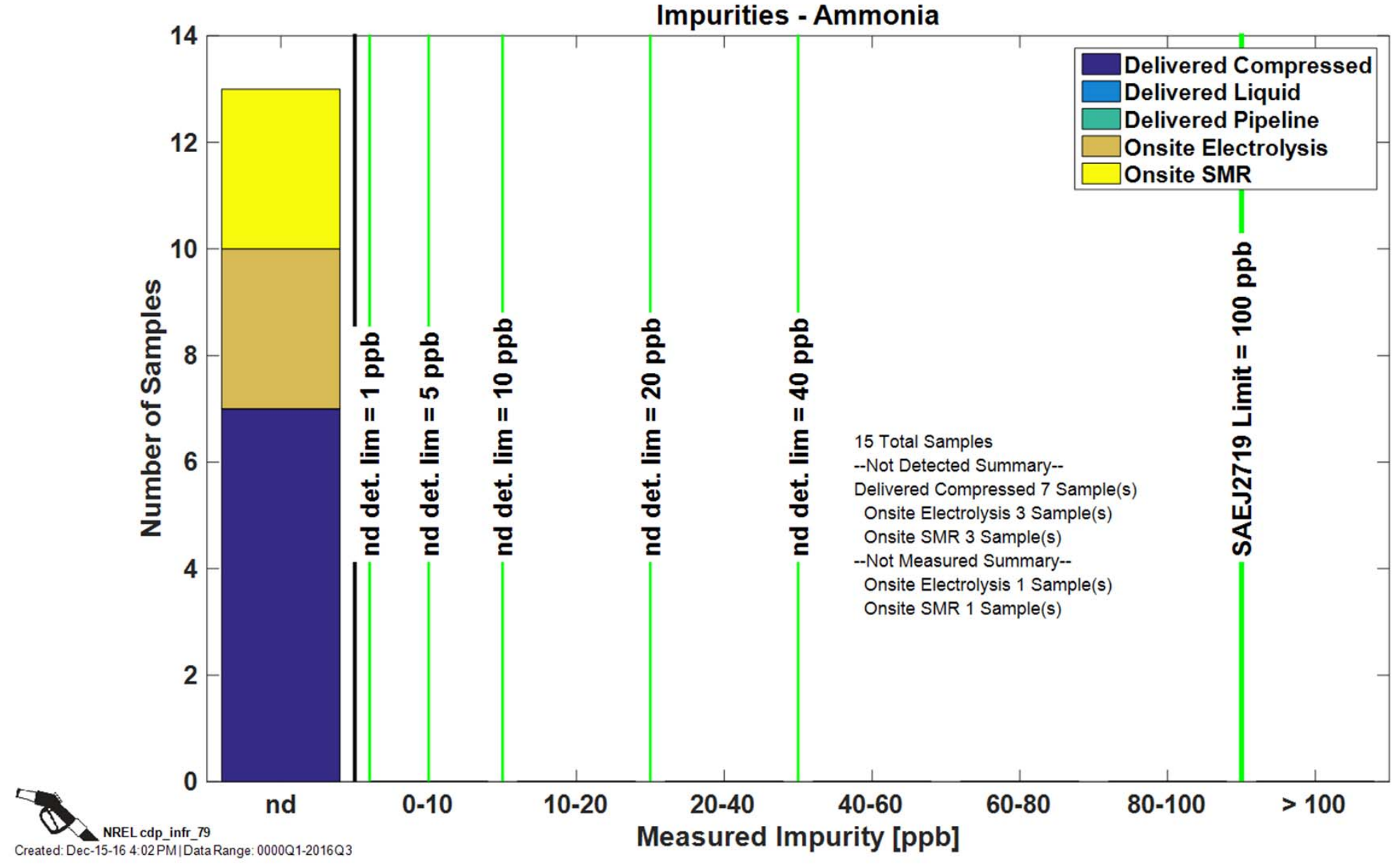




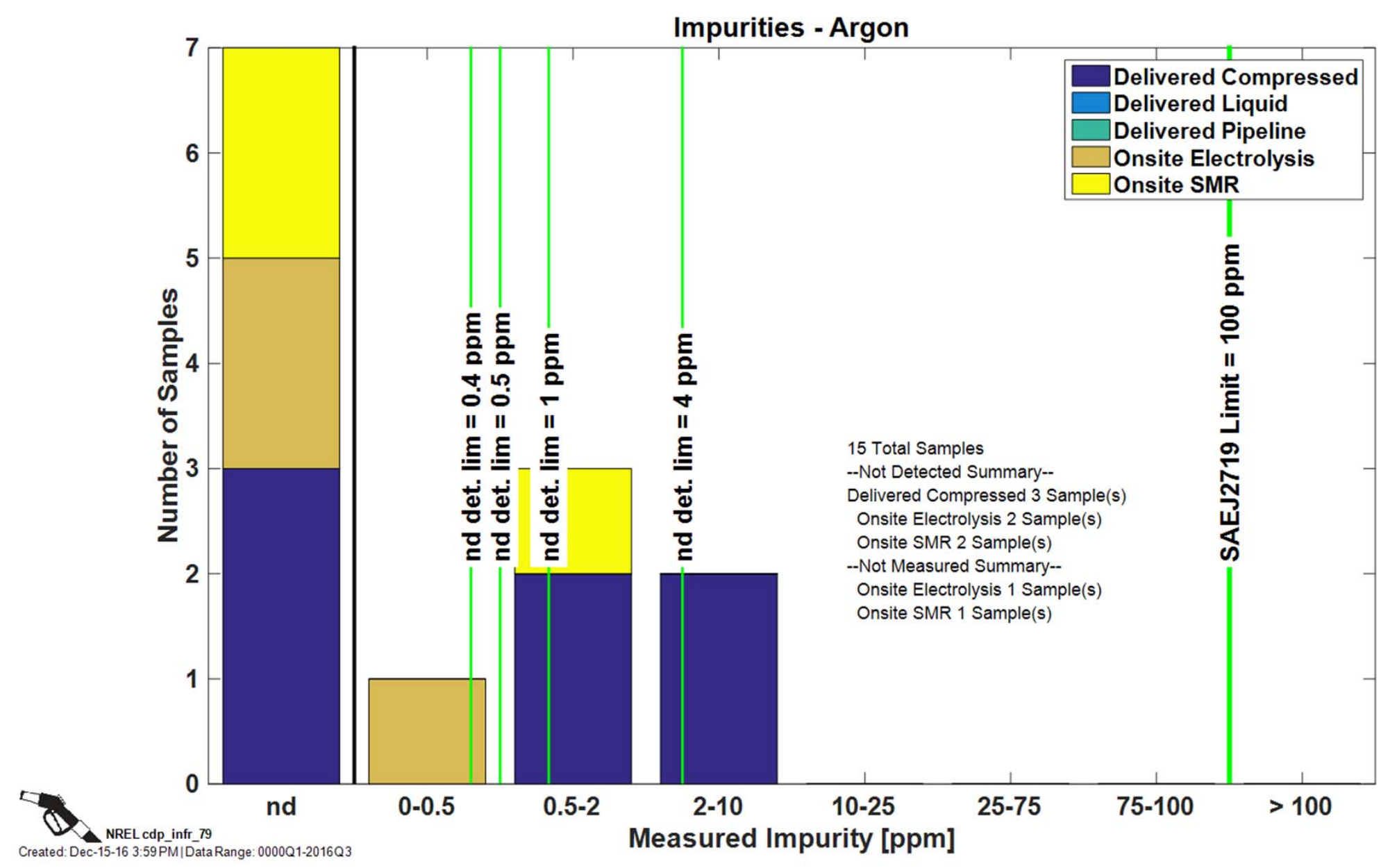




\section{Impurities-Carbon Dioxide}

Impurities - Carbon Dioxide

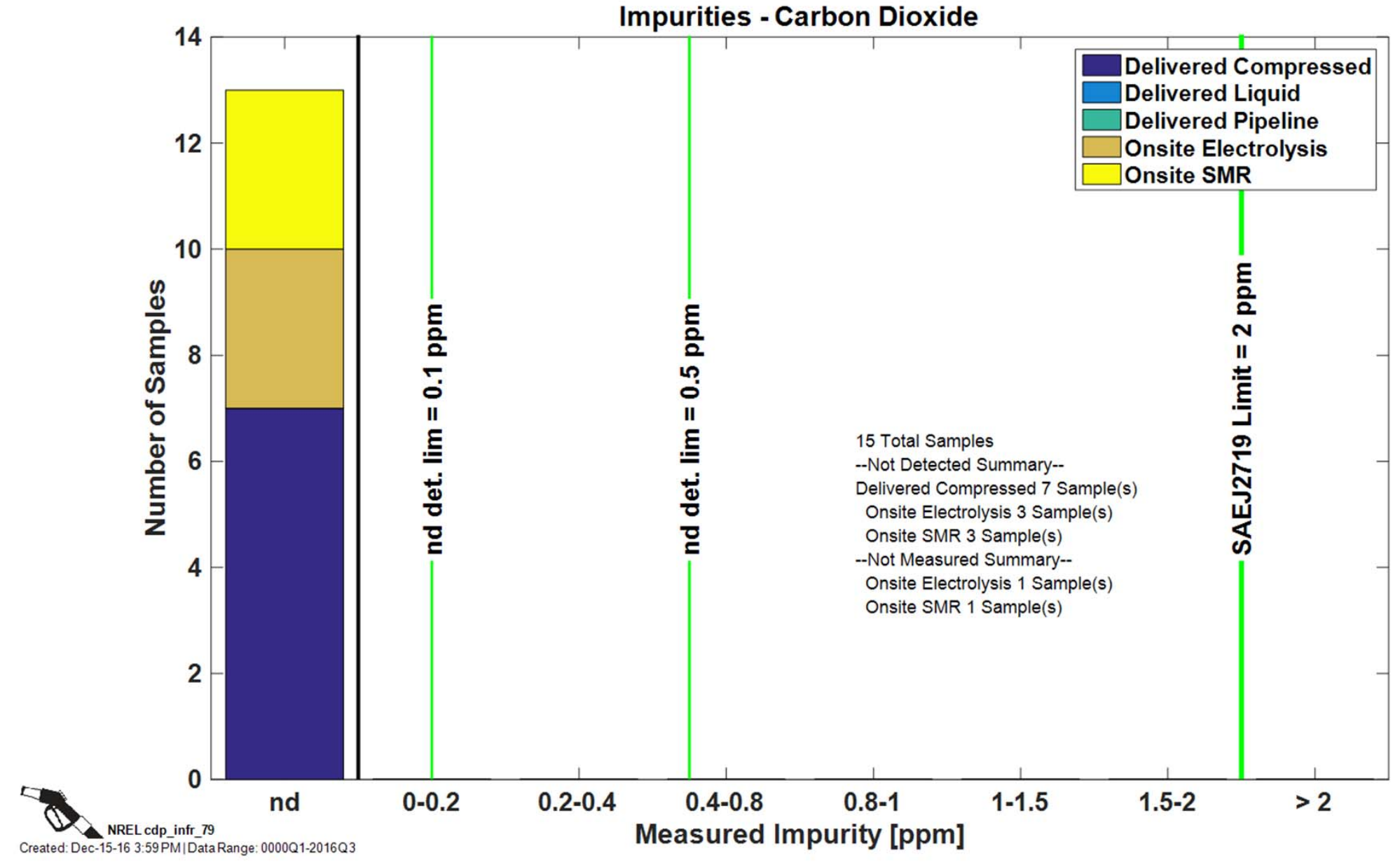




\section{Impurities-Carbon Monoxide}

Impurities - Carbon Monoxide

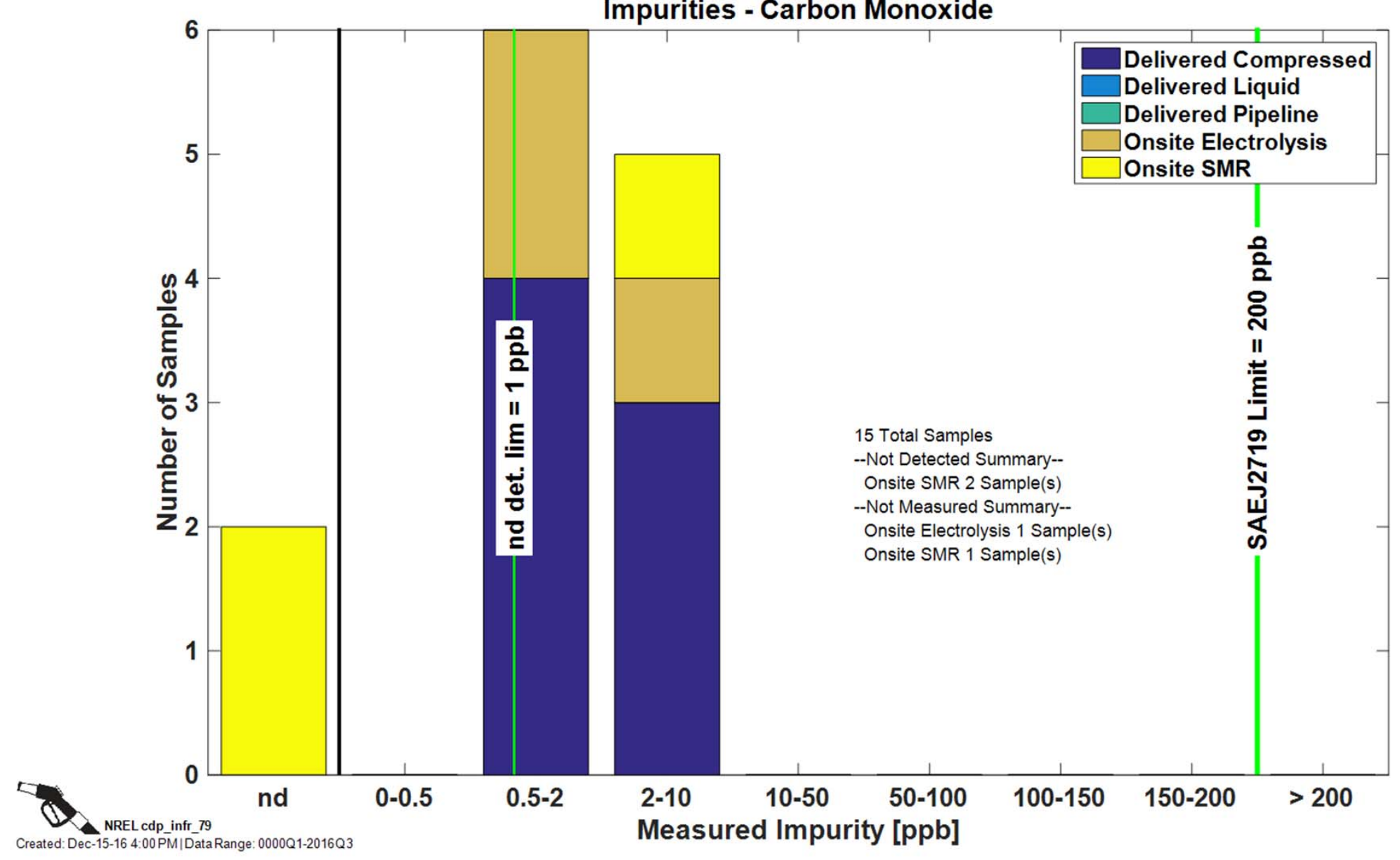




\section{Impurities-Formaldehyde}

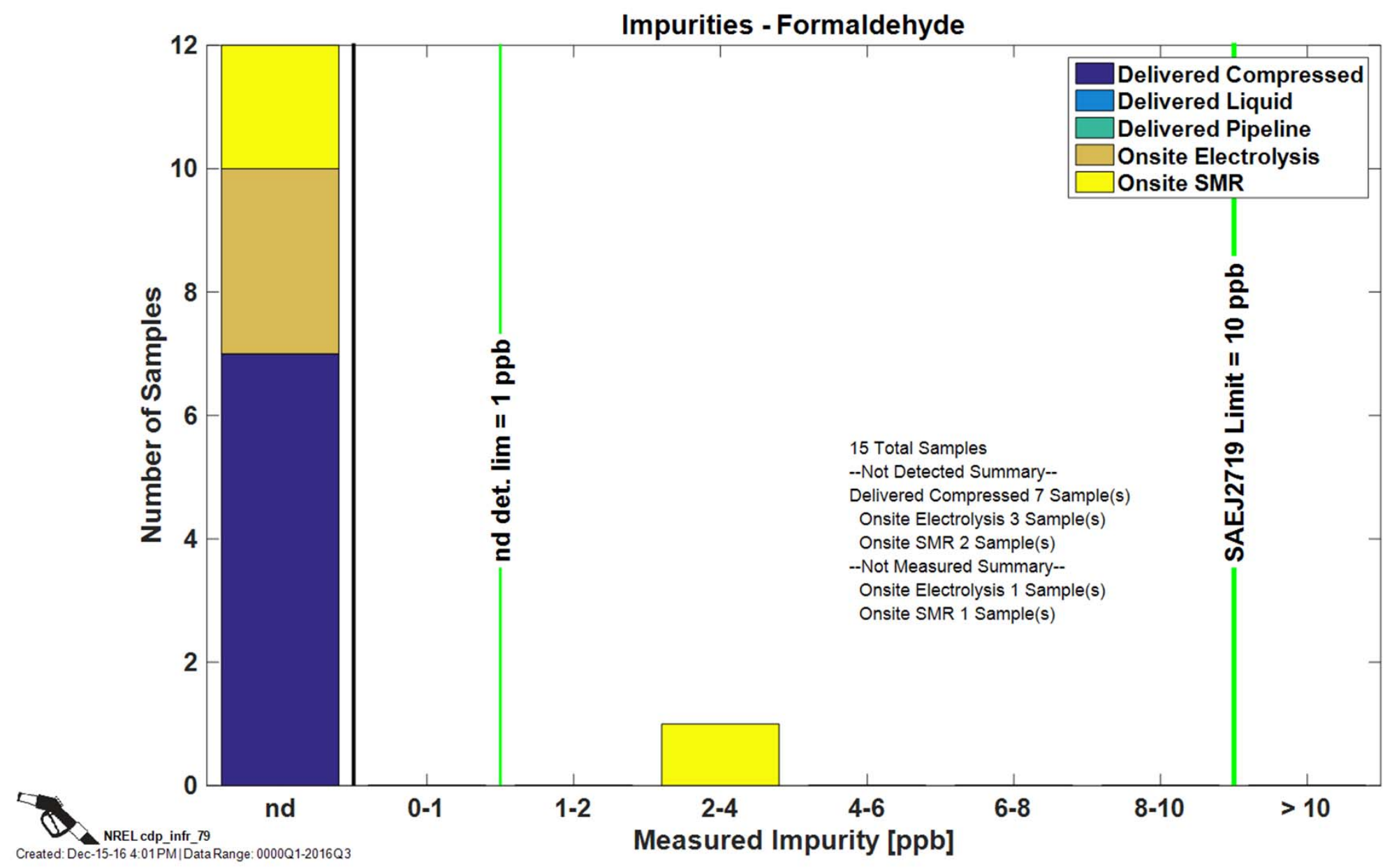




\section{Impurities-Formic Acid}

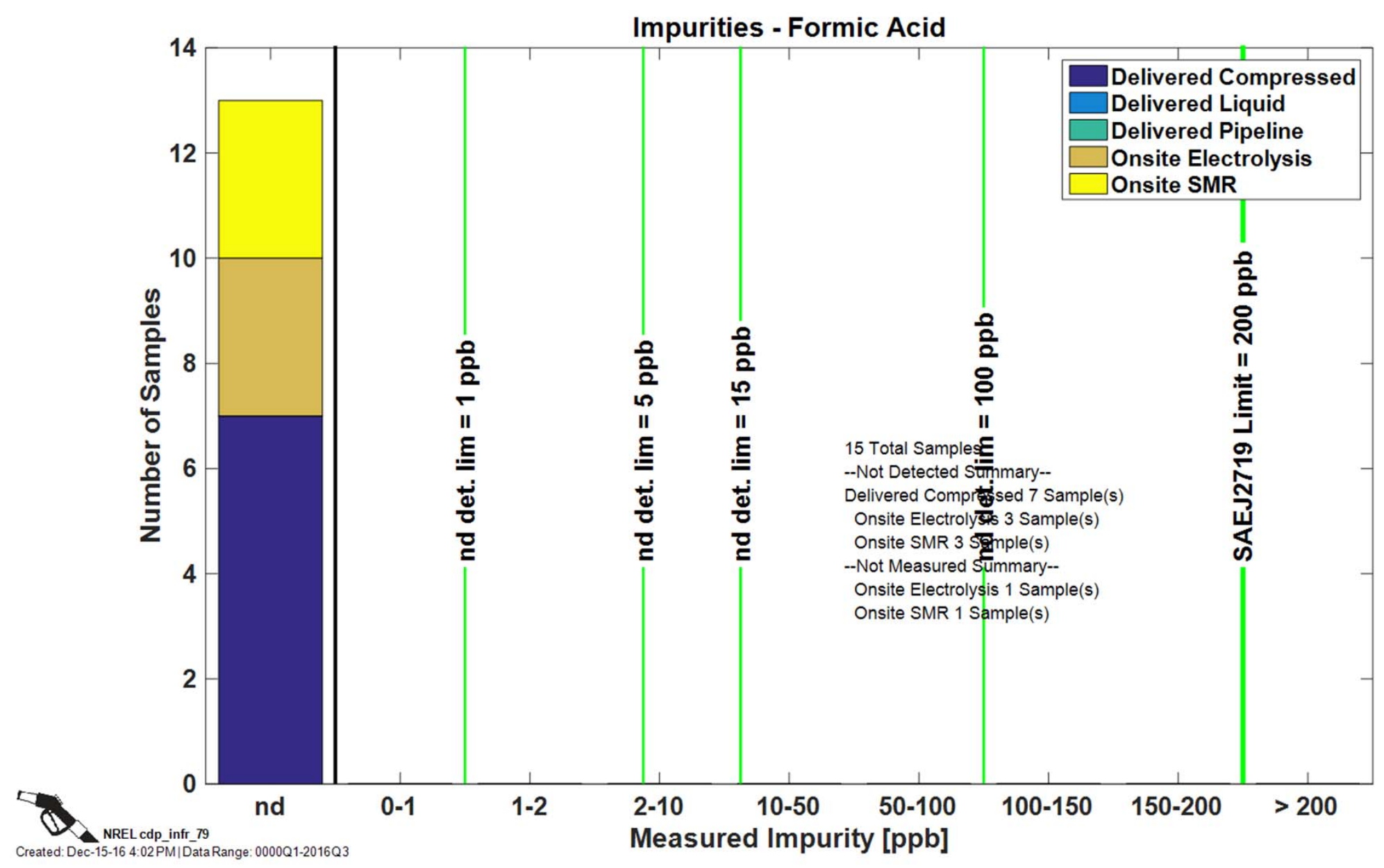




\section{Impurities-Helium}

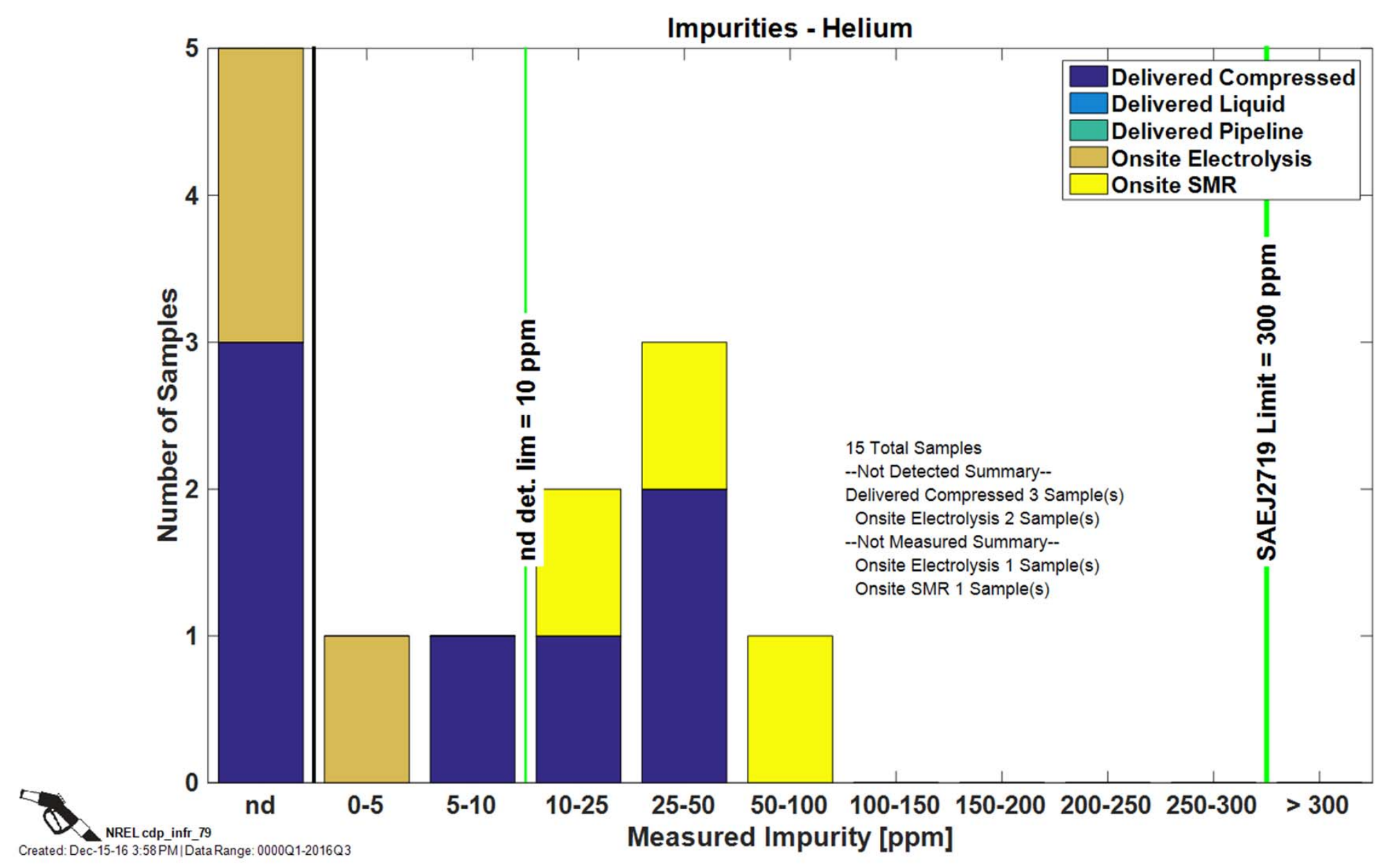




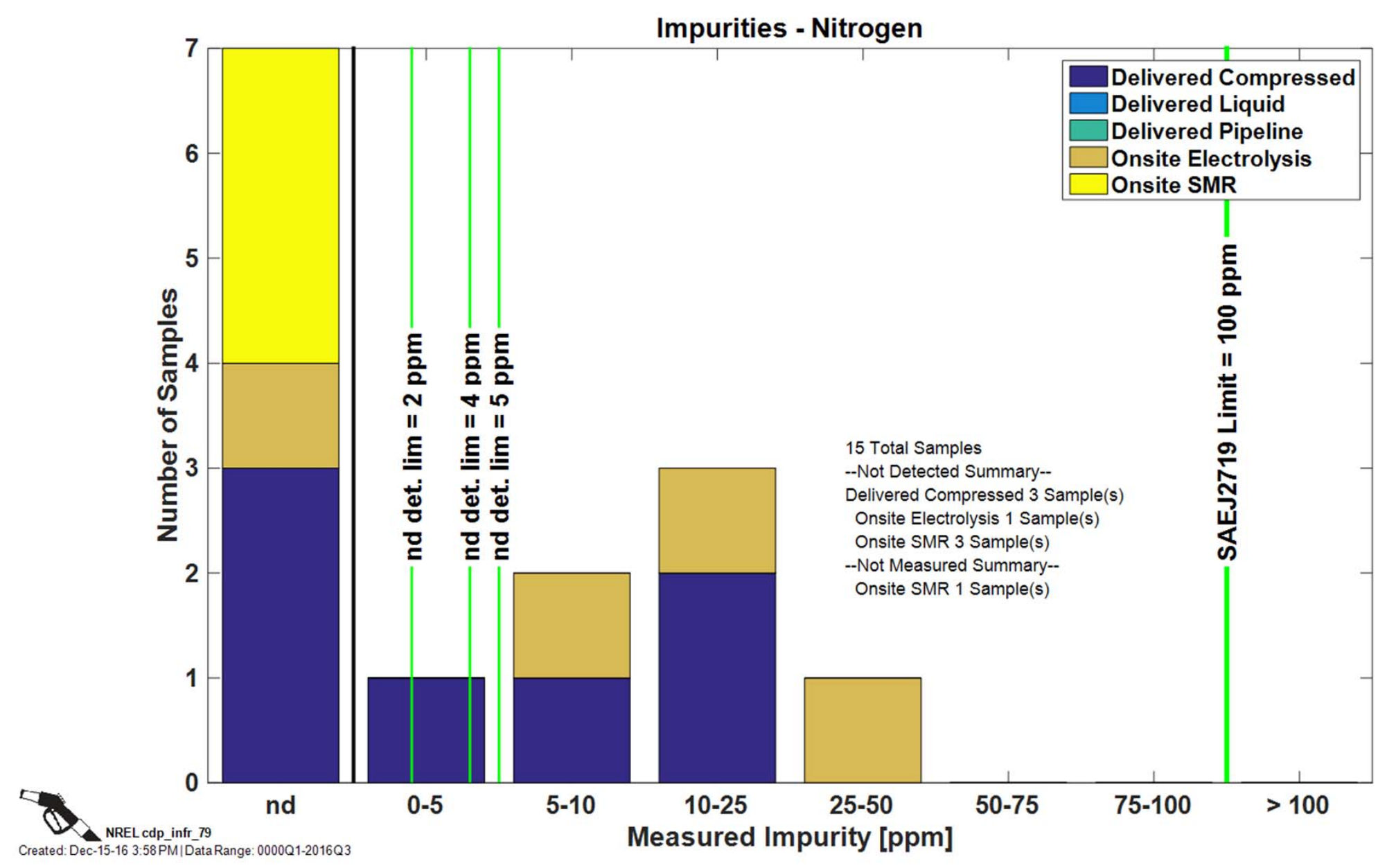




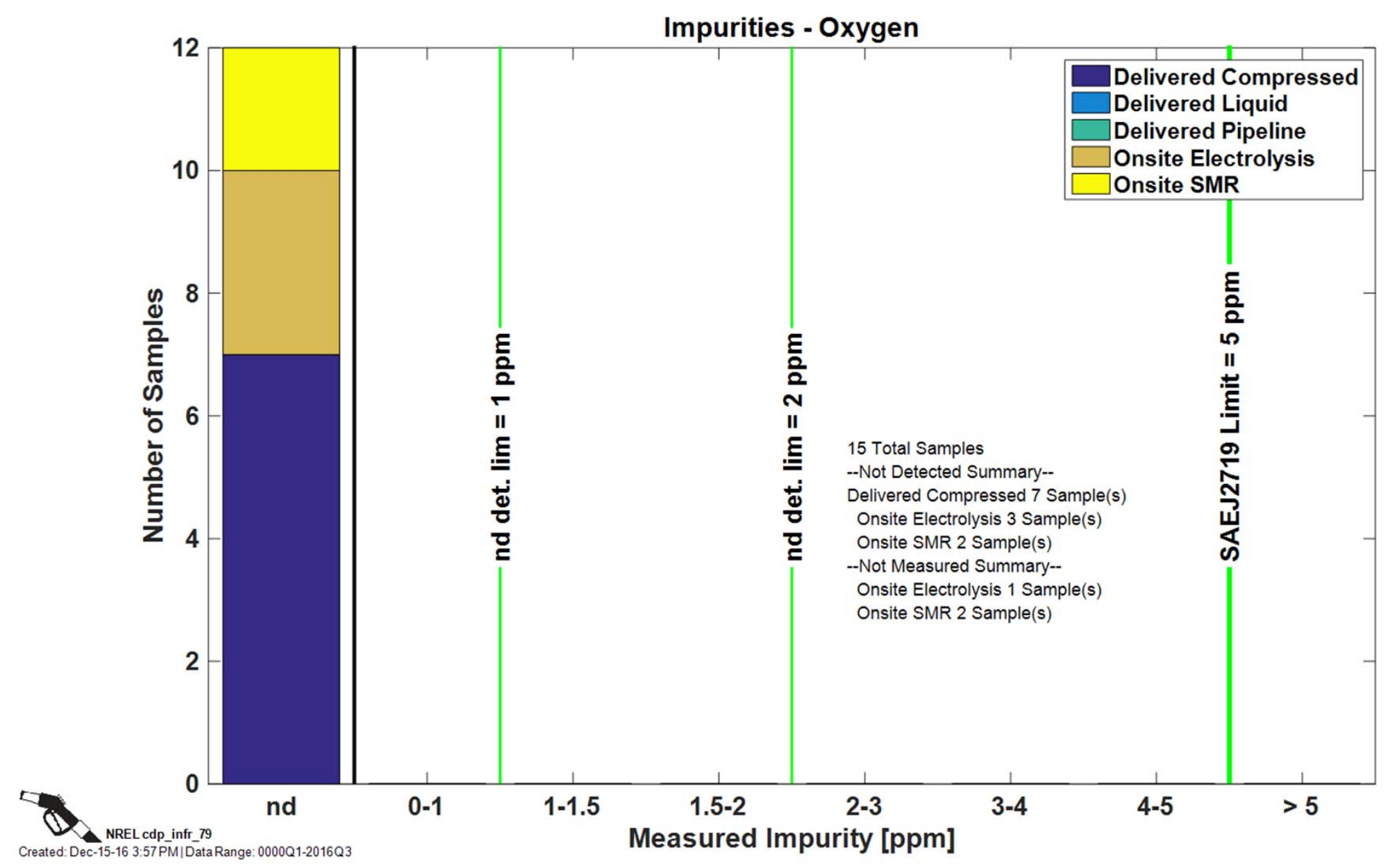




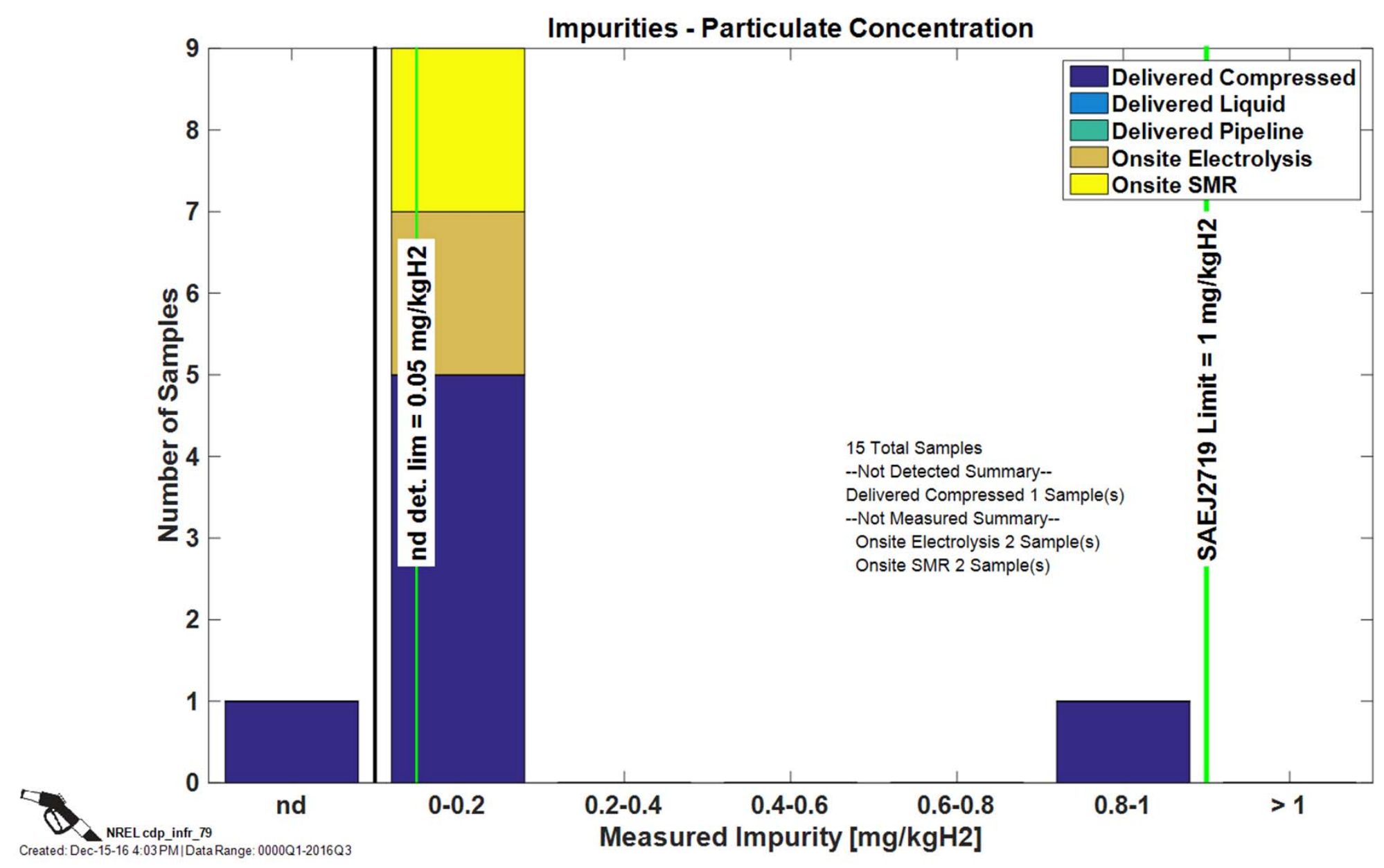




\section{Impurities-Total Halogenates}

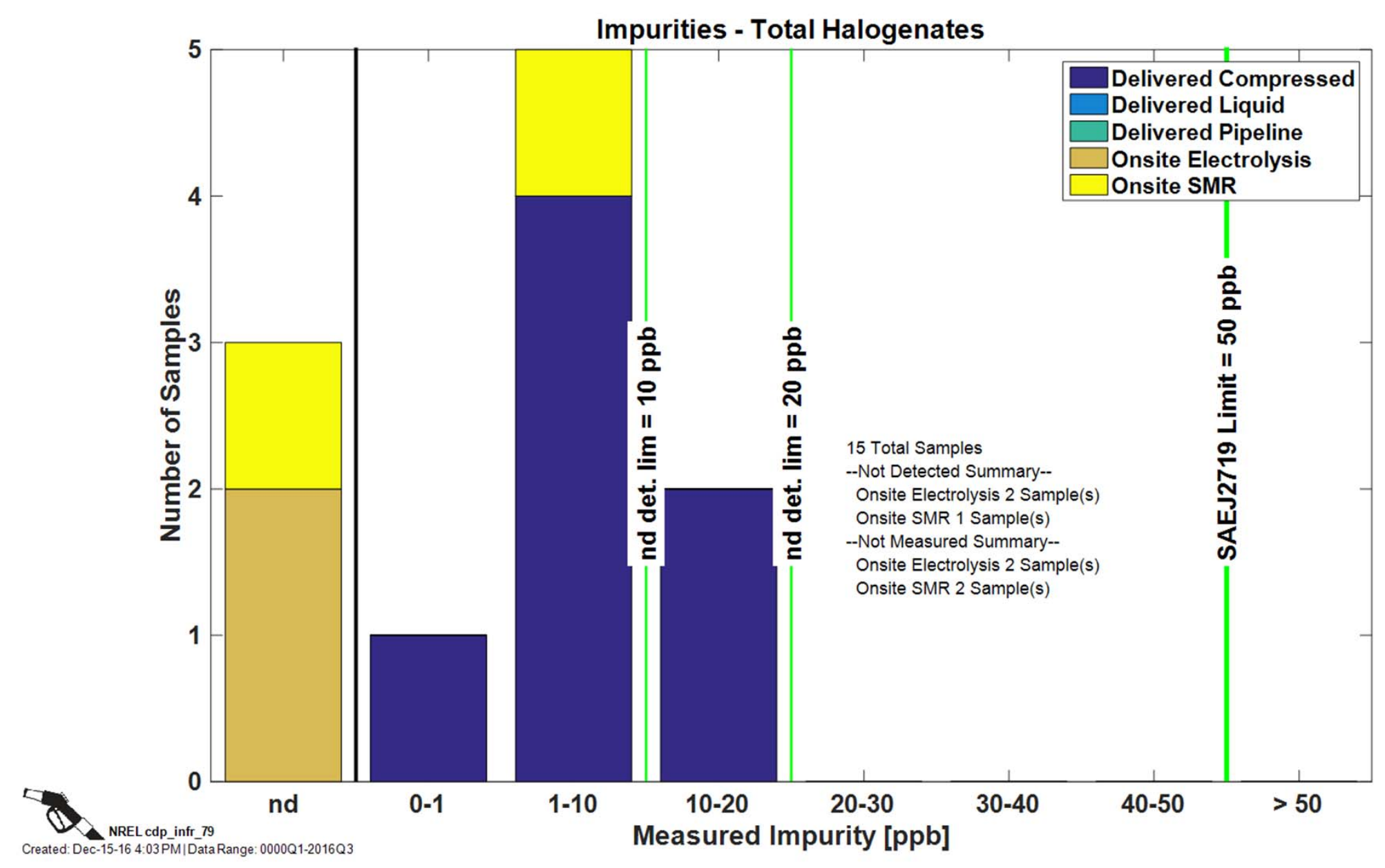




\section{Impurities-Total Hydrocarbons}

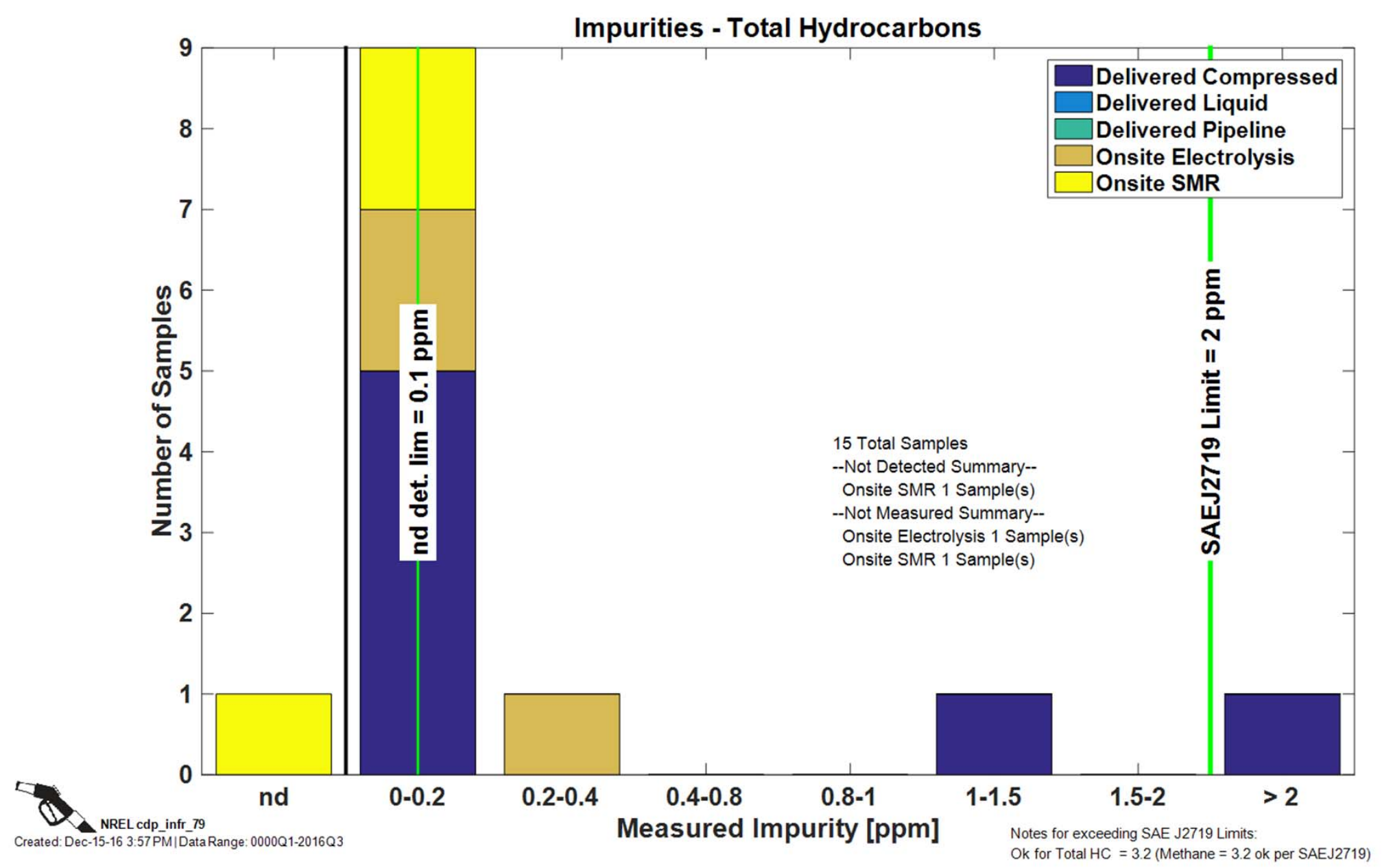




\section{Impurities-Total Sulfur}

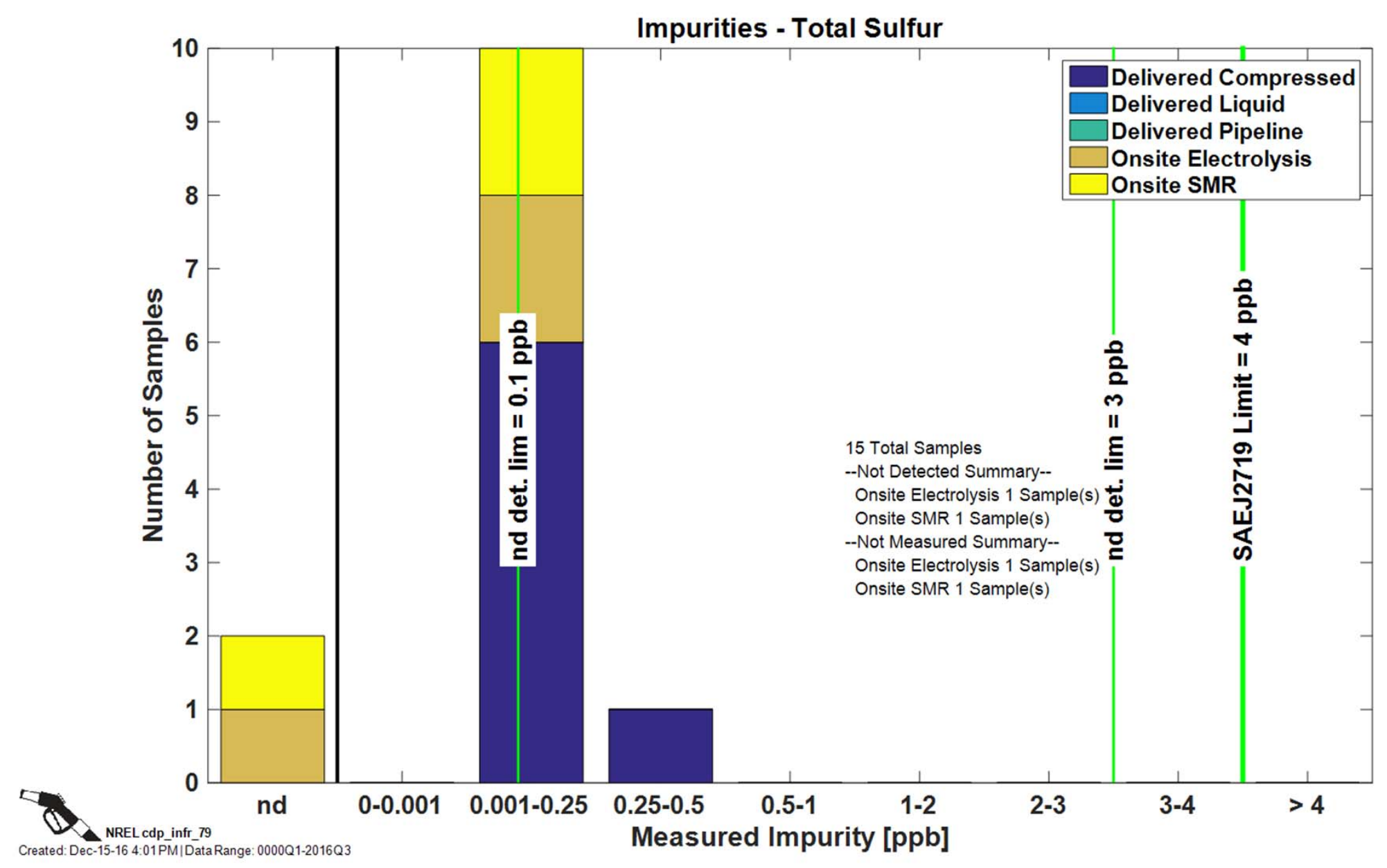




\section{Impurities-Water}

Impurities - Water

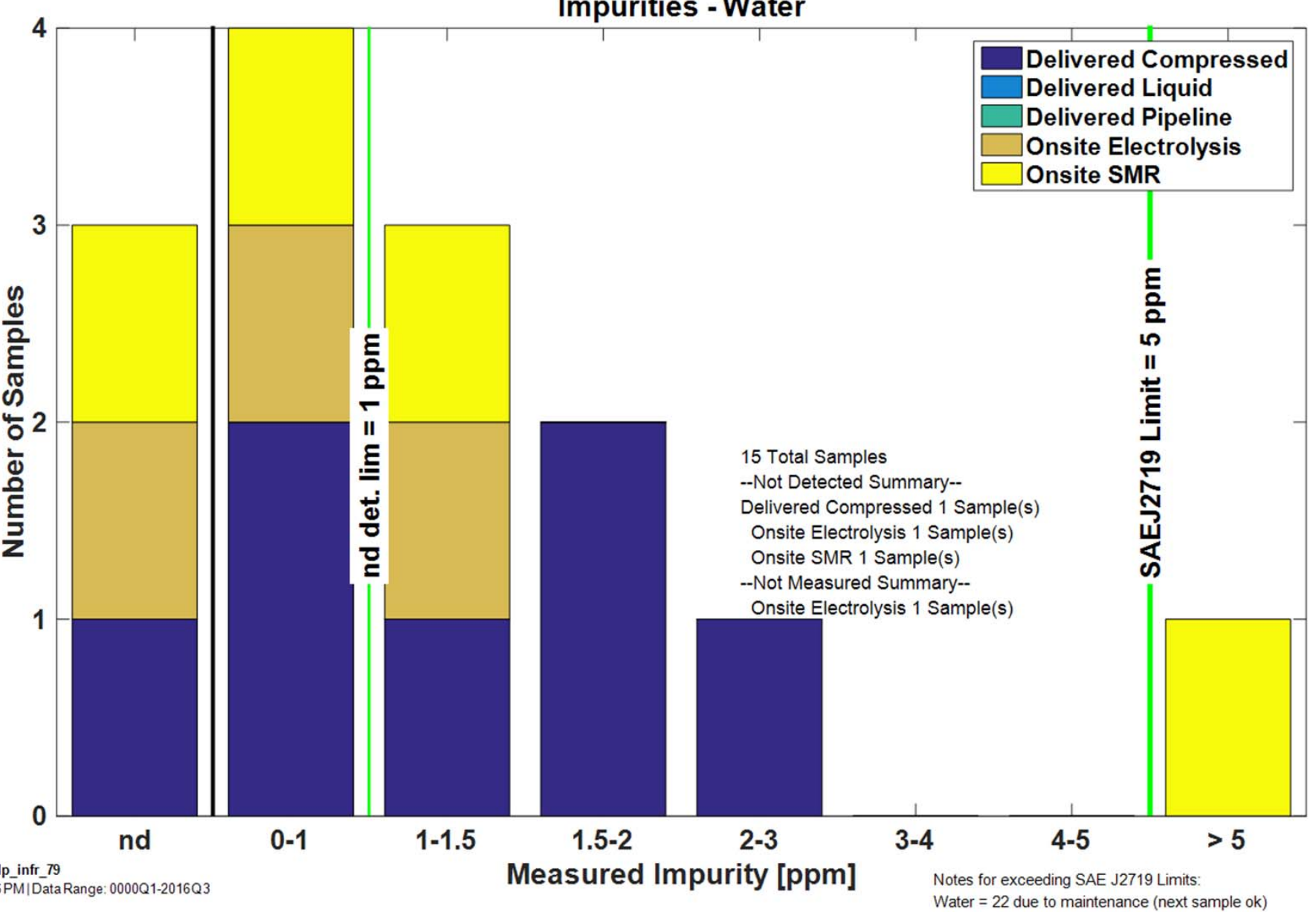




\section{Component Energy}



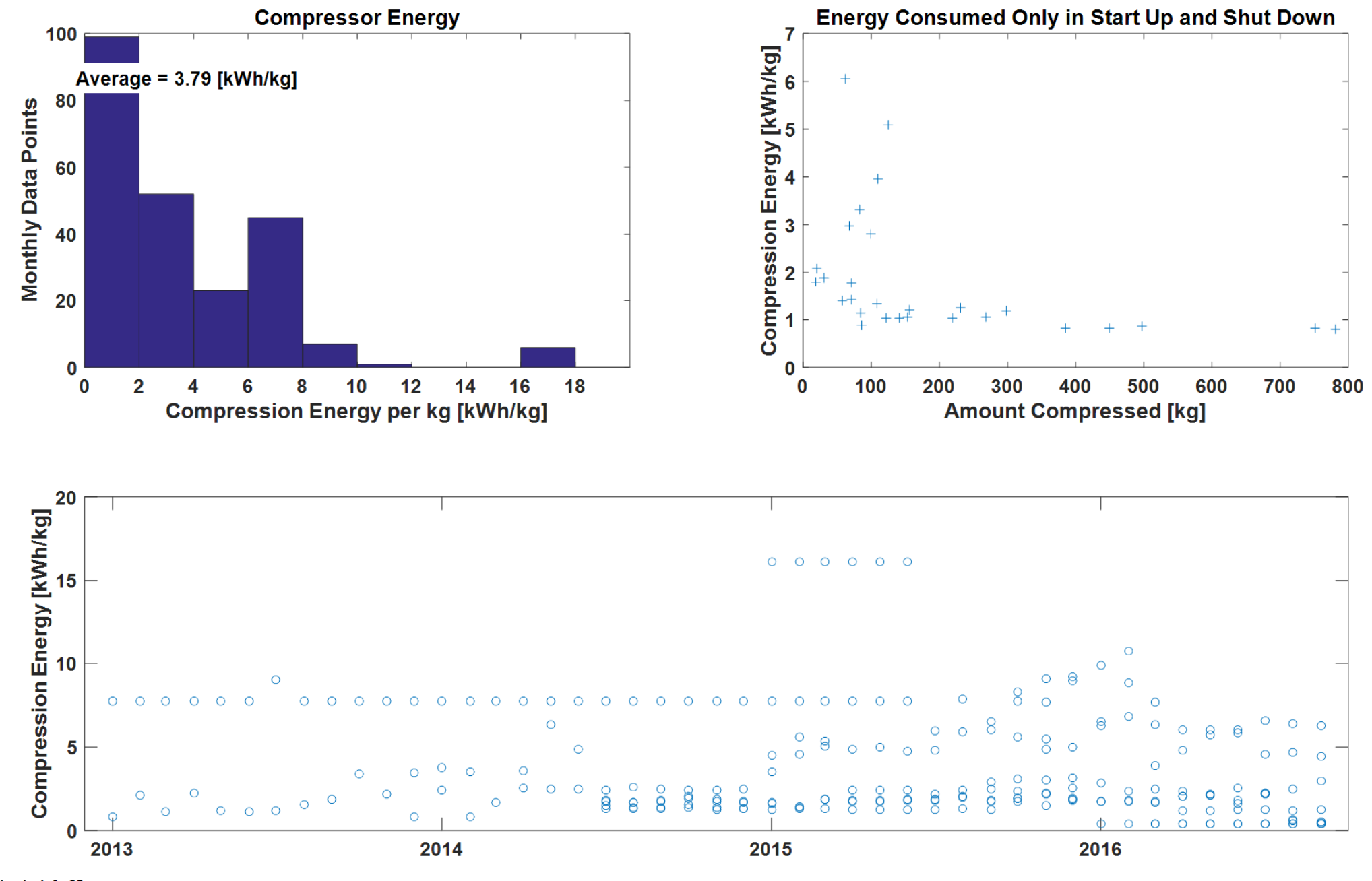

NREL cdp_infr_35

Created: Dec-21-16 11:15 AM | Data Range: 2011Q1-2016Q3 


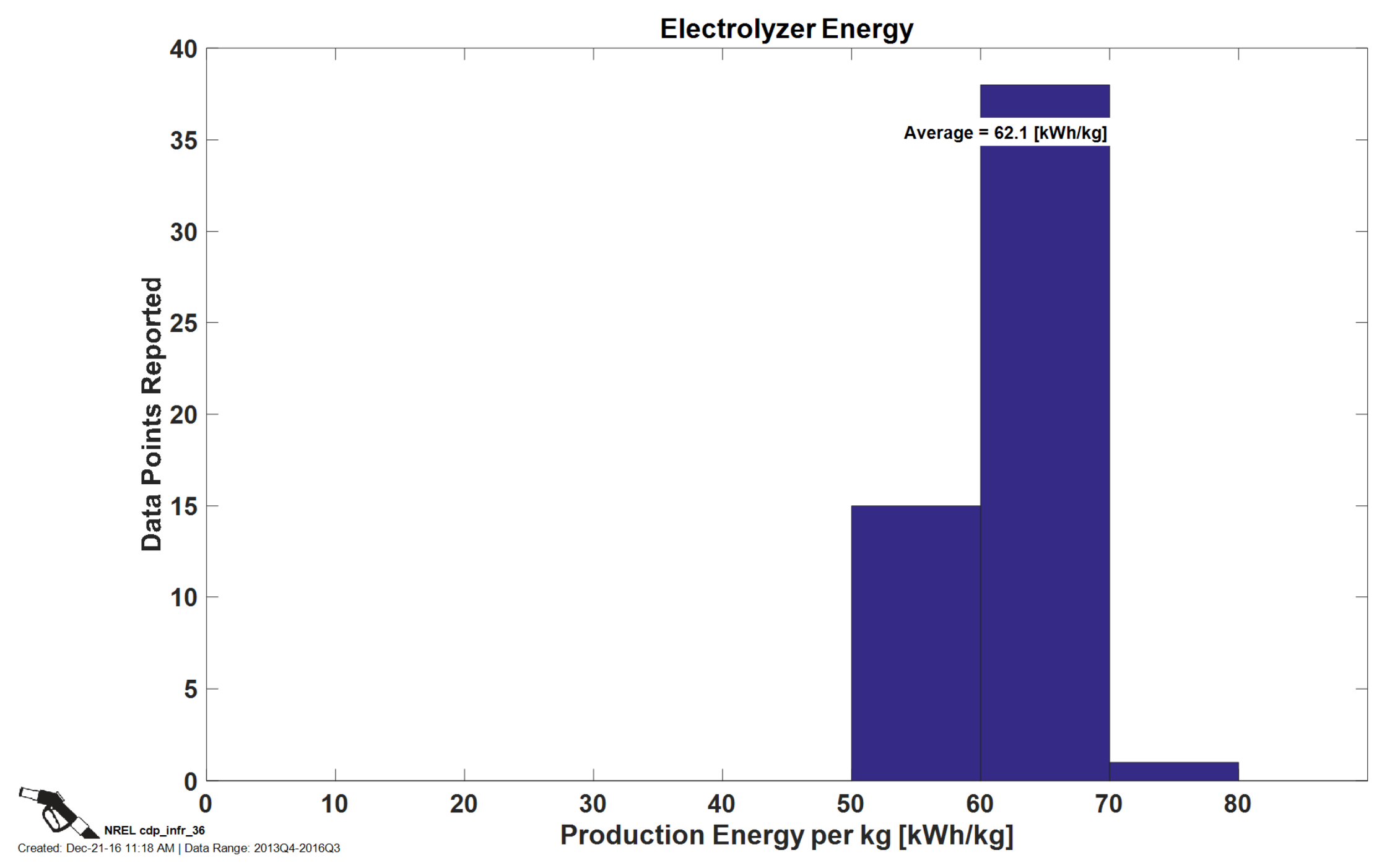




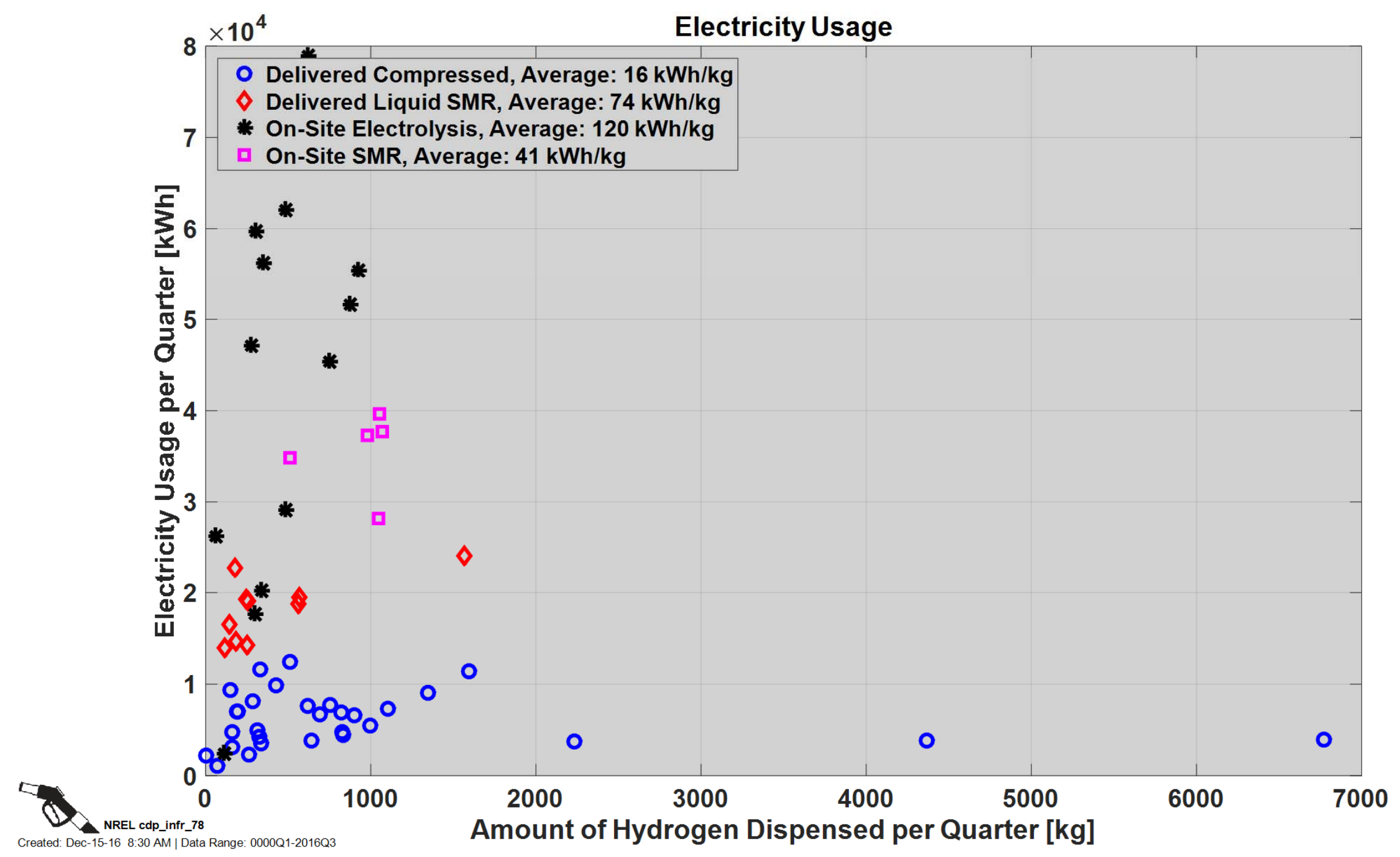

